\title{
Kryptochroma: a new genus of bark-dwelling crab spiders (Araneae, Thomisidae)
}

\author{
Miguel MACHADO ${ }^{\circledR 1, *}$, Rafaela VIECELLI ${ }^{2}$, Catherine GUZATI $^{3}$, \\ Cristian J. GRISMADO ${ }^{\circledR} 4$ \& Renato A. TEIXEIRA ${ }^{\circledR} 5$ \\ 1,2,3,5 Laboratório de Aracnologia, Escola de Ciências, Pontifícia Universidade Católica do \\ Rio Grande do Sul (PUCRS), Porto Alegre, Rio Grande do Sul, Brazil. \\ ${ }^{4}$ División Aracnología, Museo Argentino de Ciencias Naturales "Bernardino Rivadavia", \\ Buenos Aires, Argentina.
}

*Corresponding author: machadom.arachno@gmail.com

${ }^{2}$ Email: rafaviecelli1807@gmail.com

${ }^{3}$ Email: c.guzati@edu.pucrs.br

${ }^{4}$ Email: grismado@macn.gov.ar

${ }^{5}$ Email: renato.teixeira@pucrs.br

\footnotetext{
${ }^{1}$ urn:1sid:zoobank.org:author:9C99DF66-7481-4DE2-BFC2-FE73D29F8A6E

${ }^{2}$ urn:1sid:zoobank.org:author:78CA106B-2CFC-4B8B-8E05-168350DA95F8

${ }^{3}$ urn:1sid:zoobank.org:author:9A03FF9F-07D8-4F15-9735-FAC96FE23015

${ }^{4}$ urn:lsid:zoobank.org:author:A1D00976-57EF-417E-AE28-DF3BF2BAB6B1

${ }^{5}$ urn:Isid:zoobank.org:author:BA923A63-8230-40C0-819A-588EE3815CA9
}

\begin{abstract}
A recent phylogenetic analysis has shown that the genus Stephanopis comprises several different lineages of bark-dweller crab spiders. The ones with South American distribution that present a close relationship with other Neotropical genera like Epicadus, Onocolus, Rejanellus and Epicadinus were recovered in a single clade, with good support and stability. Here, we present a taxonomic review of Stephanopis species attributed to the 'pentacantha clade', proposing the new genus Kryptochroma Machado gen. nov. to accommodate them. New distribution records are provided and the following species are described for the first time: Kryptochroma gigas Machado \& Viecelli gen. et sp. nov., Kryptochroma hilaris Machado \& Teixeira gen. et sp. nov., Kryptochroma quadrata Machado \& Viecelli sp. nov. and Kryptochroma septata Machado \& Teixeira sp. nov. The species Stephanopis borgmeyeri is considered a nomen dubium, Stephanopis aheneus is a junior synonym of Epicadus tuberculatus, Stephanopis quimiliensis is transferred to Ulocymus and Stephanopis stelloides is transferred to Epicadus, being Epicadus stelloides the senior synonym of Stephanopis salobrensis, Stephanopis trilobata and Epicadus caudatus.
\end{abstract}

Keywords. Stephanopinae, taxonomy, new species, new records, Stephanopis.

Machado M., Viecelli R., Guzati C., Grismado C.J. \& Teixeira R.A. 2021. Kryptochroma: a new genus of barkdwelling crab spiders (Araneae, Thomisidae). European Journal of Taxonomy 778: 26-70.

https://doi.org/10.5852/ejt.2021.778.1565 


\section{Introduction}

The morphological aspects and taxonomic boundaries of Neotropical stephanopines have been extensively studied during the past years (Machado et al. 2015, 2018, 2019a; Silva-Moreira \& Machado 2016). Meanwhile, phylogenies based on morphological and molecular data were congruent in recovering some close related genera, corroborating classic propositions for the possible existence of tribes or even certain "groups" in this subfamily (Benjamin 2011; Benjamin et al. 2008; Wheeler et al. 2017; Machado et al. 2017, 2019b; Machado \& Teixeira 2021).

In the taxonomic review of Epicadus Simon, 1895, Silva-Moreira \& Machado (2016) highlighted that the morphological resemblances between this genus with Epicadinus Simon, 1895, Onocolus Simon, 1895 and Rejanellus Lise, 2005 could be a strong indication for a common ancestry of what the authors called the "Epicadus group", despite the absence of a phylogenetic approach to test such hypothesis. One year later, Machado et al. (2017) stated that at least two features of the male genitalia of species of the genera mentioned above could be synapomorphies for the "Epicadus group"; however, the placement of Rejanellus remained uncertain. Recently, the more inclusive approach on Australian stephanopines allowed Machado \& Teixeira (2021) to widely score genital features of several representatives of this subfamily, helping not only to elucidate the relationships in Stephanopis O. Pickard-Cambridge, 1869 but also compile more evidences that help to justify the proposition of the "Epicadus group", originally suggested by Silva-Moreira \& Machado (2016) based on taxonomic insights.

Machado \& Teixeira (2021) recovered a stable and well-supported clade of Stephanopis species that, not only emerged apart from the "Stephanopis stricto sensu", but also gathered individuals presenting two synapomorphic characters that were for the first time observed in Thomisidae (presence of ventral macrosetae on patellae I and II, and male genitalia having a pear-shaped tegulum). The authors named this group of five species the "pentacantha clade", which was recovered as sister to Rejanellus. Besides the striking morphological resemblance to Epicadus, Epicadinus, Rejanellus and Onocolus, the "pentacantha clade" has coincident geographical distribution with these four genera, known as the "Epicadus group" (Silva-Moreira \& Machado 2016). Even so, Machado \& Teixeira (2021) refrained from proposing major taxonomic acts such as new generic assignments to these species. According to the authors, the conservative morphology of species belonging to the "pentacantha clade" made it difficult to resolve the internal topology of the group, since they can only be distinguished from each other by details on their genitalia, as the curvature, size and shape of the RTA and embolus, or presence/ absence of a median septum on the epigynal plate.

The Neotropical species of Stephanopis that compose the above mentioned clade were mostly described by Mello-Leitão (1929) in his extensive work on Brazilian crab spiders. However, the illustrations and descriptions provided by this author lacks clear diagnostic characters that would allow the recognition of the taxonomic entities. Considering the compiled evidence and the need of a proper morphological study on the group, hereinafter we formally propose Kryptochroma Machado gen. nov. All species hitherto attributed to the "pentacantha clade" (sensu Machado \& Teixeira 2021) are transferred from Stephanopis to this new genus, which is diagnosed here through detailed representations of both somatic and genital features. New distribution records are provided and four new species are described.

\section{Material and methods}

\section{Repositories}

The specimens examined are deposited in the following institutions (curators in parentheses):

FMNH $=$ The Field Museum, Chicago, United States (Petra Sierwald)

IBSP $=$ Instituto Butantan, São Paulo, Brazil (Antonio D. Brescovit) 


\begin{tabular}{|c|c|c|}
\hline INPA & $=$ & zônia, Manaus, Bra \\
\hline MACN-Ar & $=$ & $\begin{array}{l}\text { Museo Argentino de Ciencias Naturales "Bernardino Rivadavia", Buenos Aires, } \\
\text { Argentina (Martín J. Ramírez) }\end{array}$ \\
\hline $\mathrm{MCN}$ & $=$ & $\begin{array}{l}\text { Museu de Ciências Naturais da Fundação Zoo-Botânica do Rio Grande do Sul, Brazil } \\
\text { (Ricardo Ott) }\end{array}$ \\
\hline MCTP & $=$ & $\begin{array}{l}\text { iências e Tecnologia da Pontifícia Universidade Católica do Rio Grande do } \\
\text { legre, Brazil (Renato A. Teixeira) }\end{array}$ \\
\hline $\mathrm{ICZ}$ & $=$ & $\begin{array}{l}\text { Museum of Comparative Zoology of Harvard, Cambridge, United States (Gonzalo } \\
\text { Giribet and Laura Liebensperger) }\end{array}$ \\
\hline LP & $=$ & ata, La Plata, Argentina (Cristina Damborenea and Luis A. Pereira) \\
\hline NHN & $=$ & $\Gamma$ \\
\hline MNRJ & $=$ & Brazil (Adriano B. Kury) \\
\hline MPEG & $=$ & eldi, Belém, Brazil (Alexandre B. Bo \\
\hline MZPW & $=$ & $\begin{array}{l}\text { of the Zoological Institute of the Polish Academy of Sciences, Warsaw, } \\
\text { rzy Prószyński) }\end{array}$ \\
\hline ZSP & $=$ & São Paulo, Brazil (Ricardo Pinto-da-Rocha) \\
\hline K & $=$ & \\
\hline OUM & $=$ & $\begin{array}{l}\text { Oxford University Museum of Natural History, Oxford, United Kingdom (Zoë M. } \\
\text { Simmons) }\end{array}$ \\
\hline 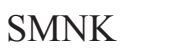 & $=$ & hes Museum für Naturkunde Karlsruhe, Germany (Hubert Höfer) \\
\hline UFMG & 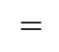 & idade Federal de Minas Gerais, Belo Horizonte, Brazil (Adalberto \\
\hline $\mathrm{Ml}$ & & eum für Naturkunde der Humboldt-Universität, Berlin, Germany (Jason Dunlop) \\
\hline
\end{tabular}

\section{Laboratory procedures and abbreviations}

All measurements provided were taken in millimeters and the terminology used to name both somatic and copulatory structures follows Machado et al. (2018). The dissection procedure applied to study the female genitalia consisted in detaching the epigynal plate from the spider body and immersing it in proteolytic enzyme (pancreatine solution). In order to accelerate the process of digestion of the soft tissues, the solution was placed in a double boiler for a few minutes. Males had their left palps removed and represented in ventral and retrolateral views. Most photographs of the habitus, front and both male and female genitalia were taken on a Multipurpose Zoom Microscope Leica M205A with a digital camera. Scanning electron microscopy images were taken at the Centro de Microscopia e Microanálises (CEMM) of the Pontifícia Universidade Católica do Rio Grande do Sul (PUCRS), where the examined material was prepared, receiving a coat of AuPd and placed in a high vacuum chamber to be properly analyzed and photographed with a microscope model Inspect F50 (FEI).

\section{Anatomical abbreviations}

$\begin{array}{ll}\mathrm{A} & =\text { atrium } \\ \mathrm{ALE} & =\text { anterior lateral eye } \\ \mathrm{AME} & =\text { anterior median eye } \\ \mathrm{CD} & =\text { copulatory duct } \\ \mathrm{CO} & =\text { copulatory opening } \\ \mathrm{EF} & =\text { epigynal fold } \\ \mathrm{FD} & =\text { fertilization duct } \\ \mathrm{MS} & =\text { median spire } \\ \mathrm{PLE} & =\text { posterior lateral eye } \\ \mathrm{PME} & =\text { posterior median eye } \\ \mathrm{RTA} & =\text { retrolateral tibial apophysis } \\ \mathrm{S} & =\text { spermatheca }\end{array}$




\title{
Results
}

\section{Taxonomy}

\author{
Class Arachnida Lamarck, 1801 \\ Order Araneae Clerck, 1757 \\ Family Thomisidae Sundevall, 1833 \\ Subfamily Stephanopinae O. Pickard-Cambridge, 1871 \\ Kryptochroma Machado gen. nov. \\ urn:1sid:zoobank.org:act:5BDEDDA5-91E4-4D7C-A632-C305D02748FD
}

\section{Type species}

Stephanopis pentacantha Mello-Leitão, 1929. Designated here.

\section{Diagnosis}

The species of Kryptochroma gen. nov. resemble some of those of Epicadus [e.g., Epicadus caudatus (Mello-Leitão, 1929) and Epicadus tuberculatus (Petrunkevitch, 1910)] by their cryptic bark-dwelling habitus with predominant brown or reddish-brown body coloration, and those of Epicadinus, especially by their small size (varying from approximately three millimeters of the total body length for males and nine to females) and spiny habitus (body covered by many setae and rugose teguments, and opisthosoma bearing stout conical projections) (Fig. 2A-D). However, they can be easily recognized and distinguished from Epicadus, Epicadinus and other genera of Stephanopinae by the presence of a pair of ventral macrosetae on their anterior patellae (I and II) (Fig. 1D), sensorial pits (three to five trichobothria surrounded by a small group of duster-shaped setae) on dorsal tibial depressions preceded by a strong plumose macroseta (Fig. 1C), and a pair of circular taints on the posterior slope of the prosoma (Figs 3A, 4A, 7A). Differently from Epicadinus, the species of Kryptochroma gen. nov. have five opisthosomal projections instead of three, and short leaf-shaped setae covering their entire body instead of long needle-shaped ones (Fig. 1A-B). Females present a flat epigynal plate, short copulatory ducts and a single pair of oval spermathecae (Fig. 10C-F); the male palp bears a pear-shaped tegulum with a short and fixed embolus, and a single tipped, stout and conical RTA (Fig. 11C-F).

\section{Etymology}

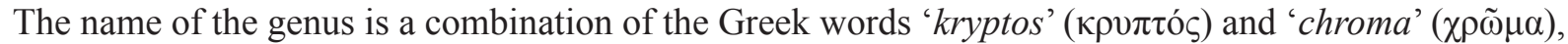
which respectively mean 'hidden' and 'color'. The name is a reference to the general color pattern of these spiders, which is associated to their cryptic behavior. Gender feminine.

\section{Description}

Prosoma. Granular surfaced due to the presence of spherical setae sockets (Fig. 1A-B); slightly longer than wide, pear-shaped and usually covered with organic particles. ALE almost two times as large as AME, prosoma coloration shows a gradient of brown and dark-yellow shades (predominantly reddish brown in Kryptochroma hilaris Machado \& Teixeira gen. et sp. nov.), lighter on the posterior slope, where there is a pair of rounded spots close to the anterior border of the opisthosoma (Figs 3A, 4A, 7A); in some cases the individual has a yellow longitudinal line between the MS and PME (Figs 3A, 5A, 7A, 9A). Anterior eye row strongly recurved, AME close to each other, and posterior eye row procurved (Fig. 11A-B). Clypeus with a central pair of serrated macrosetae (Fig. 1A). Sternum scutiform, slightly longer than wide in females and as long as wide in males, with brush-shaped setae. Carapace with a conical medianposterior projection pointing backwards (except for K. hilaris gen. et sp. nov. and K. parahybana (MelloLeitão, 1929) gen. et comb. nov., which have just an obtuse and slight elevation on the top of the thoracic area); opisthosoma short with five stout projections (two lateral pairs and one single and larger terminal 

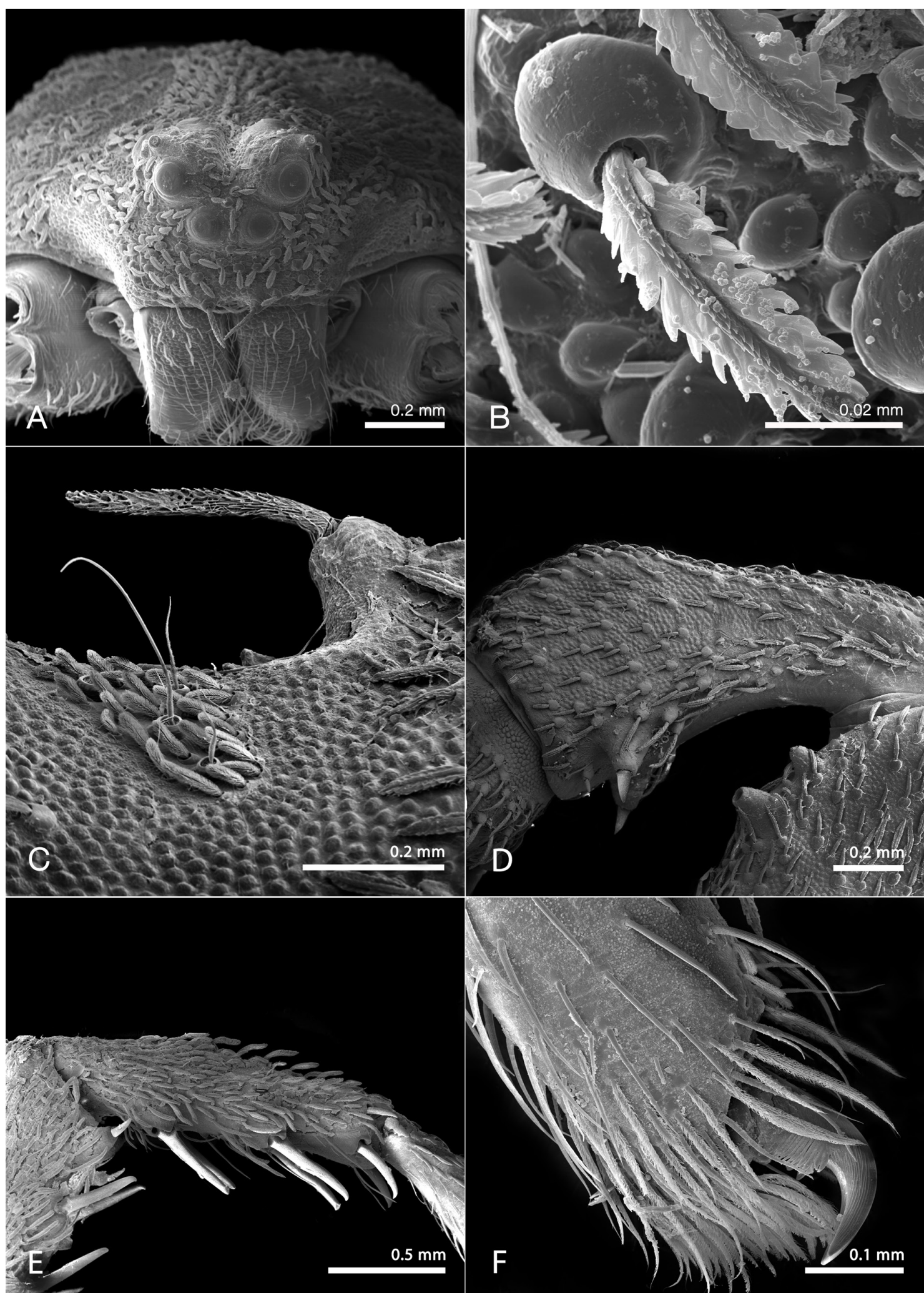

Fig. 1. Scanning electron microscopy of a male of Kryptochroma pentacantha (Mello-Leitão, 1929) gen. et comb. nov. (MCTP 7362). A. Prosoma, frontal view. B. Detail of a leaf-shaped setae and its spherical socket. C. Sensorial pit preceeded by a plumose macrosetae on tibia I. D. Pair of ventral macrosetae on patella I. E. Metatarsal macrosetae. F. Tarsal claw and detail of setae tuft. 
projection), anal region and spinneret ring area projected backwards. Mouthparts: chelicerae with five equal-sized teeth; three on the promarginal row and two on the retromarginal. Labium truncated, slightly wider than long. Endites truncated, longer than wide, with scarce promarginal scopula.

LEGS. Anterior femora enlarged and bearing many conical setiferous tubercles, legs I and II stouter and longer than legs III and IV, femora I and II with dorsal and dorsolateral stout macrosetae in conical sockets. Tibia I and II with five pairs of ventral macrosetae, metatarsi I and II with three ventral and one dorso-distal pair (Fig. 1E). Tarsal claws curved, pectinated, unequal in number of teeth (mesial claw with numerous thin teeth and ectal having three stout ones); subungueal tufts scarce, with brush-shaped setae (Fig. 3B).

Opisthosoma. Five short conical projections (two dorsolateral consecutive pairs, and a single caudal one), straight or slightly concave anterior border and rough surfaced, covered by leaf-shaped setae (Fig. 1B). Anal region and spinneret ring projected backwards, elongated.
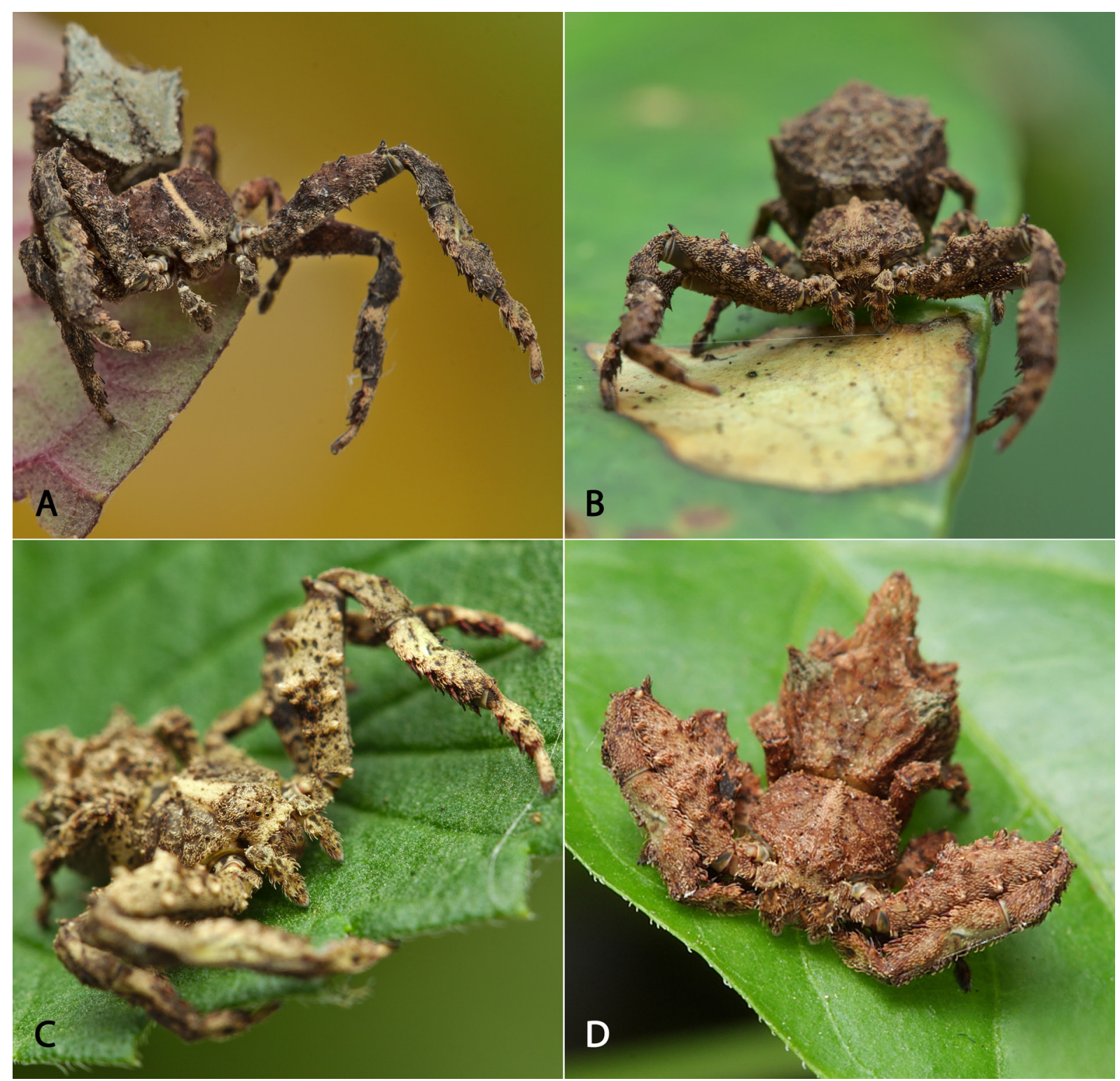

Fig. 2. A-D. Color variations of live specimens of Kryptochroma Machado gen. nov. Photo credits: Thiago Carvalho. 
Genitalia. Palpus with cymbium and tegulum pear-shaped, without apophyses; RTA single tipped, stout and presenting grooves at the apex (Figs 8D, 11D, 13D). The internal morphology of female genitalia is very conservative, presenting a single pair of reniform spermathecae without any accessory glands or coiled ducts (Figs 3D, 4D, 7D, 10D, 12D, 16D); in ventral view the copulatory openings are excavated in relation to the rest of the surface of the genital area, separated by a septum and located close to the edges of the posterior margin of the epigynal plate (Figs 10C, 12C, 16C).

\section{Composition}

Nine species distributed within the Neotropical Region: Kryptochroma gigas Machado \& Viecelli gen. et sp. nov., Kryptochroma hilaris Machado \& Teixeira gen. et sp. nov., Kryptochroma macrostyla (MelloLeitão, 1929) gen. et comb. nov., Kryptochroma parahybana (Mello-Leitão, 1929) gen. et comb. nov., Kryptochroma pentacantha (Mello-Leitão, 1929) gen. et comb. nov., Kryptochroma quadrata Machado \& Viecelli gen. et sp. nov., Kryptochroma quinquetuberculata (Taczanowski, 1872) gen. et comb. nov., Kryptochroma renipalpis (Mello-Leitão, 1929) comb. nov. and Kryptochroma septata Machado \& Teixeira gen. et sp. nov.

\section{Distribution}

Brazil (Amazonas, Bahia, Espírito Santo, Minas Gerais, Pará, Paraíba, Paraná, Pernambuco, Roraima, Rio de Janeiro, Rio Grande do Sul, Santa Catarina, and São Paulo) and French Guiana (Cayenne, Maripasoula, Saint-Élie and Saül) (Figs 9, 18).

\section{Variations}

Each one of the color variations documented here (from greyish-green to dark-yellow or predominantly reddish-brown), as well as the stained (e.g., opisthosoma of $K$. septata sp. nov. - Fig. 16A) and striped patterns (e.g., prosoma of the specimen in the Fig. 2A) were observed in all species. Therefore, since all species of Kryptochroma gen. nov. can present any combination of tegument hue, is it virtually impossible to identify them solely based on their color.

Kryptochroma gigas Machado \& Viecelli gen. et sp. nov.

urn:1sid:zoobank.org:act:6DC79758-A014-4D6F-960B-4C63ACB17982

Figs 3, 9, 21A

\section{Diagnosis}

The female of Kryptochroma gigas gen. et sp. nov. resembles those of K. quadrata sp. nov. and $K$. pentacantha by its large body size, which is significantly larger than in the other species here described. Kryptochroma gigas gen. et sp. nov. can be distinguished by the elevated and short opisthosoma and by the heart-shaped epigynal plate with a short and stout projection over a wide median septum, which divides the median field in two longitudinal gutters reaching the copulatory openings (Figs 3C-F, 21 A).

\section{Etymology}

The epithet is a Latin feminine noun that means giant, referring to the bigger size of this species compared to other species of the genus.

\section{Material examined}

Holotype

BRAZIL • $O$; Minas Gerais, Simonésia, Estação Biológica da Mata do Sossego; 2004'25" S, 4204'13" W; 28-30 Nov. 2010; M.T.T. Santos leg.; UFMG 4734.

\section{Additional material}

None. 


\section{Description}

Female (holotype, UFMG 4734)

Prosoma. Anterior eye row very recurved and posterior slightly procurved; prosoma dark yellow with large lateral black taints, median region and posterior slope yellow, contoured by black lines (Fig. 3AB). Chelicerae dark yellow with black stains, endites and labium black, sternum black with a median yellow taint.

LEGS. Femora I and II dorsally enlarged and with many setiferous tubercles, femora III and IV bicolor, half black, half yellow.

Opisthosoma. Predominantly dark-yellow, black on the back of the abdominal projections and on the sides, rough surfaced and slightly concave on the anterior border (Fig. 3A-B).

Measurements. Eye diameters and eye interdistances: AME 0.09, ALE 0.14, PME 0.12, PLE 0.12, AMEAME 0.08, AME-ALE 0.12, PME-PME 0.24, PME-PLE 0.06, MOQ length 0.43, MOQ width 0.48; leg formula: $1-2-4-3$ : leg I - femur $4.66 /$ patella 2.59 / tibiae $3.10 /$ metatarsus $2.17 /$ tarsus $1.29 /$ total 13.81; II - 3.83/ 2.07/ 2.84/ 1.86/ 1.29/ 11.89; III - 2.07/ 1.29/ 1.86/ 1.19/ 0.82/ 7.23; IV - 2.59/ $1.19 / 1.86 / 1.29 / 0.82 / 7.75$. Total body length 9.62 ; prosoma 4.40 length, 4.04 width; opisthosoma

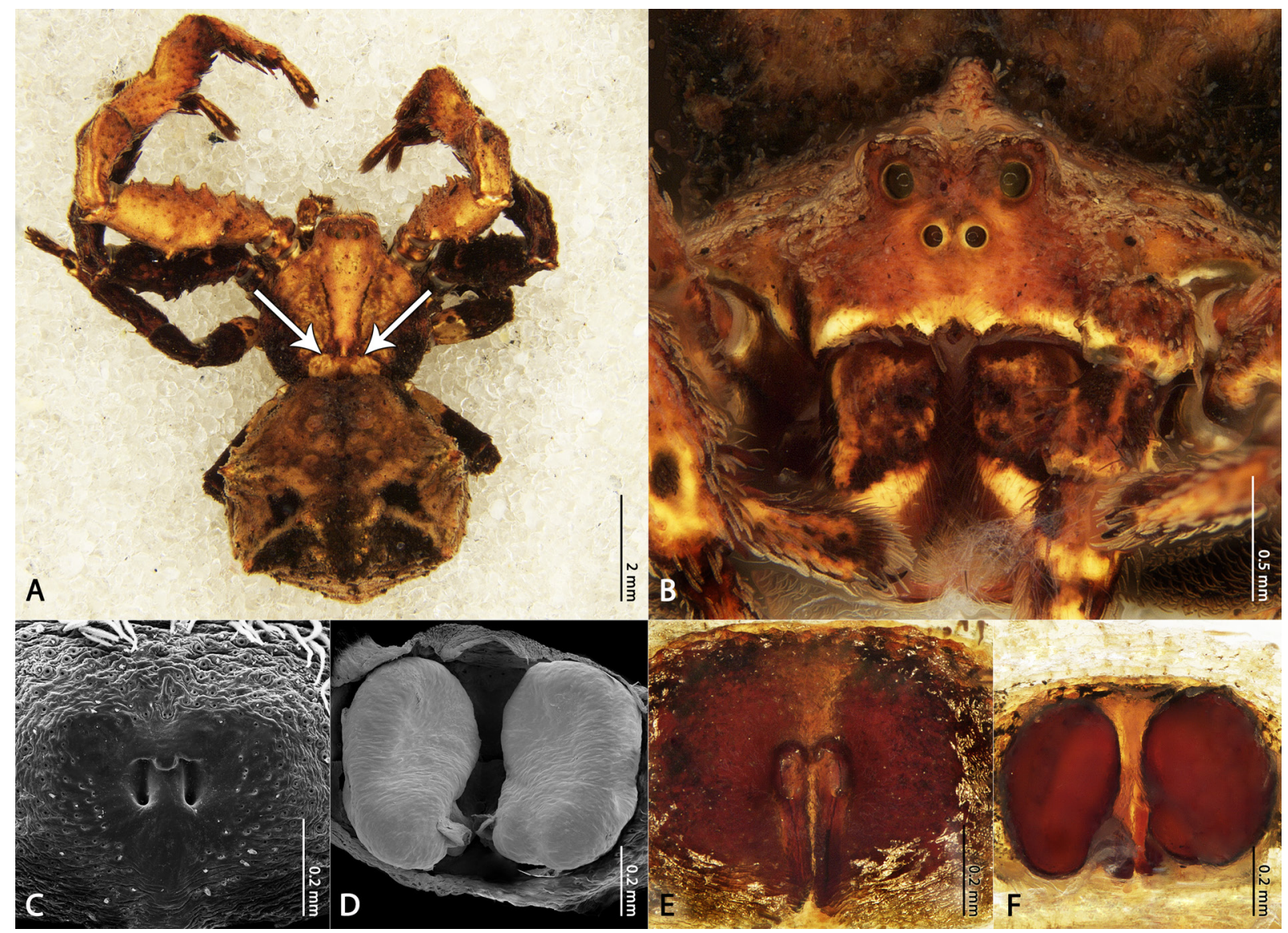

Fig. 3. Female of Kryptochroma gigas Machado \& Viecelli gen. et sp. nov. Holotype (UFMG 4734). A. Habitus, dorsal view (arrows indicate the pair of circular taints on the posterior slope of the prosoma). B. Front. C, E. Epigynal plate, ventral view. D, F. Spermathecae, dorsal view. 
length 4.62; clypeus 0.37 height; sternum 1.45 length, 1.20 width; endites 0.69 length, 0.40 width; labium 0.48 length, 0.53 width.

\author{
Male \\ Unknown.

\section{Distribution} \\ Central Brazil (Minas Gerais) (Fig. 9).
}

Kryptochroma hilaris Machado \& Teixeira gen. et sp. nov. urn:lsid:zoobank.org:act:803AFFBD-9E9F-41B4-948D-2C427AFBD9B5

Figs $4-5,9,20 \mathrm{~B}, 21 \mathrm{~B}$

\title{
Diagnosis
}

The male of K. hilaris gen. et sp. nov. has a body coloration that resemble that of K. parahybana. However, it can be distinguished by the stouter, shorter and round embolus, which points retro-ventrally (Figs 5C, E, 20B). The most striking differences between K. hilaris gen. et sp. nov. and other species of the genus are the discrete MS on prosoma, that is more like an median elevation on the thoracic area (absent in males), and the number of ventral macrosetae on tibiae I and II: while other species have four pairs on each leg for both sexes, in K. hilaris sp. nov. the females bear six ventral macrosetae on tibia I (Fig. 4A) while males present only five (Fig. 5A). Females can also be distinguished by the presence of two anterolateral and one median depression on the epigynal plate and by the copulatory openings located in a demilune-shaped concavity delimited by the posterior folds of the tegument (Figs 4C, E, 21B).

\section{Etymology}

The specific name - a Latin adjective that means cheerful - is a reference to the shape of the posterior fold of the epigynal plate, which resembles a smiling face (Figs 4C-F, 21B).

\section{Material examined}

\section{Holotype}

BRAZIL • +; Amazonas, Coari, Porto Urucu, Base de Operações Geólogo Pedro de Moura; 0448'41" S, 6502'01" W; Jul. 2006; S.C. Dias leg.; MPEG 13322.

\section{Paratypes}

BRAZIL • 1 \%; same collection data as for holotype; Sep.-Nov. 2006; N.F. Lo Man Hung leg.; MCTP 42643 • 1 + ; same collection data as for holotype; 13 Jul. 2003; D. Guimarães leg.; MCTP 42642 • 1 \%; same collection data as for holotype; Sep. 2006; C.A.C. Santos Jr. leg.; MPEG 13311.

\section{Additional material}

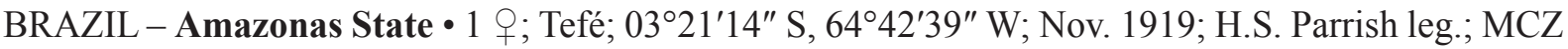

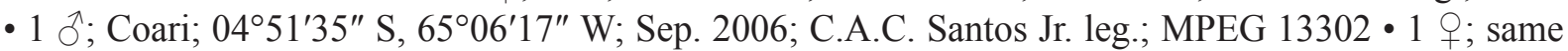
locality as for preceding; $04^{\circ} 53^{\prime} 45^{\prime \prime} \mathrm{S}, 65^{\circ} 19^{\prime} 11^{\prime \prime} \mathrm{W}$; Oct. 2006; J.O. Dias leg.; MPEG $13307 \bullet 1$ \%; same locality as for preceding; $04^{\circ} 48^{\prime} 56^{\prime \prime}$ S, $65^{\circ} 01^{\prime} 53^{\prime \prime}$ W; Jul. 2006; C.A.C. Santos Jr. leg.; MPEG 13318 • $1 \mathrm{~J}^{\lambda}$; same locality as for preceding; $04^{\circ} 50^{\prime} 01^{\prime \prime} \mathrm{S}, 65^{\circ} 03^{\prime} 53^{\prime \prime}$ W; Sep. 2006; D.F. Candiani leg.; MPEG 13354 • 1 \%; same locality as for preceding; $04^{\circ} 52^{\prime} 47^{\prime \prime}$ S, $65^{\circ} 20^{\prime} 09^{\prime \prime}$ W; Oct. 2006 ; N.F. Lo-Man-Hung leg.; MPEG 13363 • 1 q; same locality as for preceding; 04 $52^{\prime} 07^{\prime \prime} \mathrm{S}, 65^{\circ} 15^{\prime} 53^{\prime \prime} \mathrm{W} ; 13 \mathrm{Jul}$. 2003; D. Guimarães leg.; MPEG 22795 • 1 đ ; same locality as for preceding; $04^{\circ} 51^{\prime} 35^{\prime \prime}$ S, $65^{\circ} 06^{\prime} 17^{\prime \prime}$ W; Sep. 2006; S.C. Dias leg.; MPEG $13306 \bullet 10^{\lambda}$; same locality as for preceding; $04^{\circ} 52^{\prime} 36^{\prime \prime} \mathrm{S}, 65^{\circ} 09^{\prime} 05^{\prime \prime} \mathrm{W} ; 14$

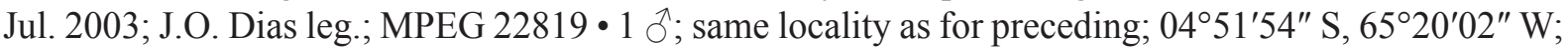


Oct. 2006; N.F. Lo-Man-Hung leg.; MPEG 13239 • 1 ô; same collection data as for preceding;

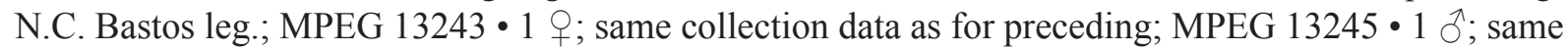

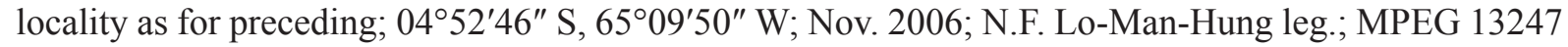

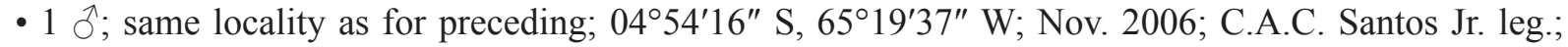

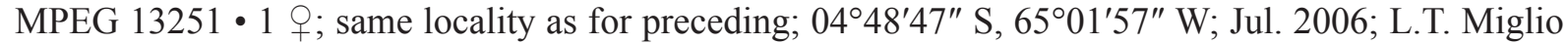
leg.; MPEG 13253 • 1 ' ; same locality as for preceding; $04^{\circ} 51^{\prime} 35^{\prime \prime} \mathrm{S}, 65^{\circ} 06^{\prime} 17^{\prime \prime} \mathrm{W}$; Sep. 2006; N.F.

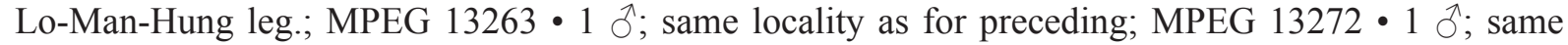

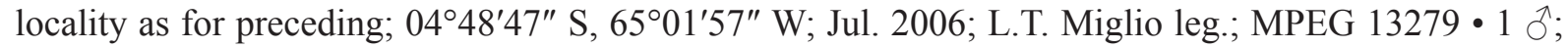

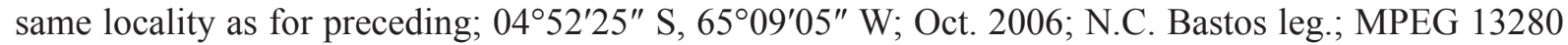

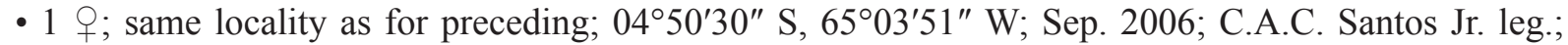

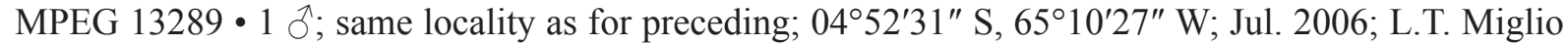
leg.; MPEG $13290 \bullet 1$ क; same locality as for preceding; $04^{\circ} 53^{\prime} 30^{\prime \prime}$ S, $65^{\circ} 20^{\prime} 07^{\prime \prime} \mathrm{W}$; Nov. 2006; N.F. LoMan-Hung leg.; MPEG 13298 • 1 o ; same locality as for preceding; $04^{\circ} 48^{\prime} 41^{\prime \prime} \mathrm{S}, 65^{\circ} 02^{\prime} 01^{\prime \prime} \mathrm{W}$; Jul. 2006; J.O. Dias leg.; MPEG 13299 • 1 ' ; same locality as for preceding; $04^{\circ} 52^{\prime} 06^{\prime \prime}$ S, $65^{\circ} 15^{\prime} 52^{\prime \prime}$ W; Jul. 2006; L.T. Miglio leg.; MPEG 13304 • 1 क; same locality as for preceding; $04^{\circ} 48^{\prime} 56^{\prime \prime} \mathrm{S}, 65^{\circ} 01^{\prime} 53^{\prime \prime} \mathrm{W}$; Jul 2006; J.O. Dias leg.; MPEG 13317 • 1 q; same locality as for preceding; $04^{\circ} 51^{\prime} 38^{\prime \prime}$ S, $65^{\circ} 20^{\prime} 04^{\prime \prime}$ W; Oct. 2006; N.F. Lo-Man-Hung leg.; MPEG $13321 \bullet 1$ + ; same locality as for preceding; $04^{\circ} 52^{\prime} 06^{\prime \prime}$ S,

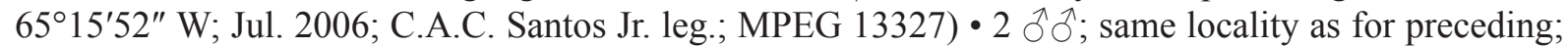
$04^{\circ} 48^{\prime} 45^{\prime \prime}$ S, 6501'58" W; Jul. 2006; J.O. Dias leg.; MPEG $13329 \bullet 1$ q; same locality as for preceding;

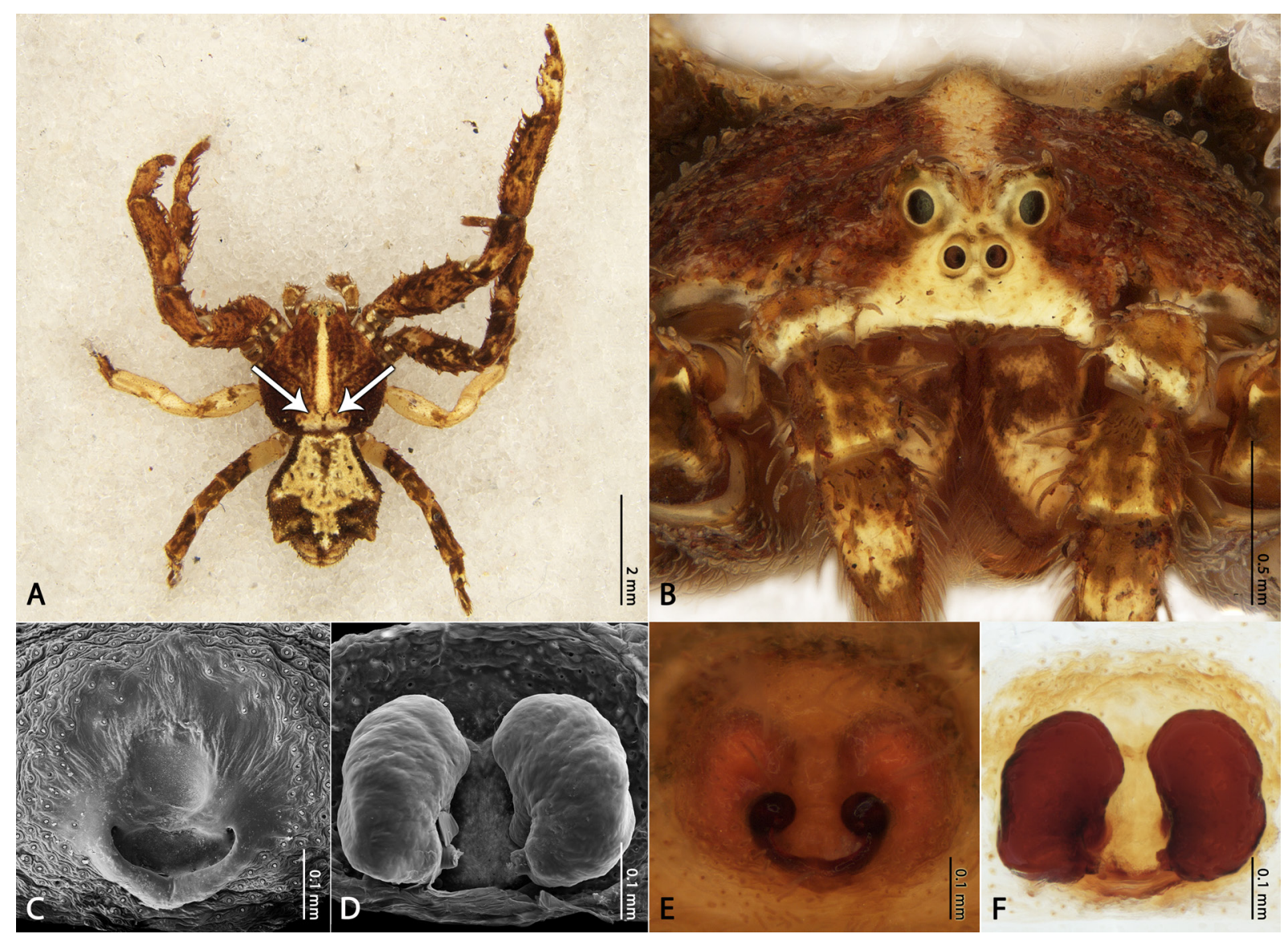

Fig. 4. Female of Kryptochroma hilaris Machado \& Teixeira gen. et sp. nov. Holotype (MPEG 13322). A. Habitus, dorsal view (arrows indicate the pair of circular taints on the posterior slope of the prosoma. B. Front. C, E. Epigynal plate, ventral view. D, F. Spermathecae, dorsal view. 
0453'14" S, 65¹3'37" W; Sep. 2006; J.O. Dias leg.; MPEG 13337 • 1 ô, 1 juv.; same locality as for

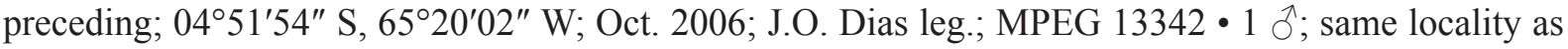

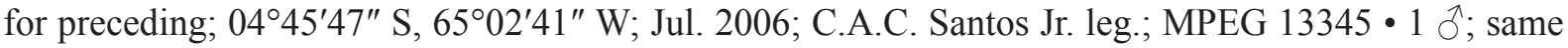
locality as for preceding; 04 $50^{\prime} 32^{\prime \prime}$ S, $65^{\circ} 04^{\prime} 08^{\prime \prime}$ W; Sep. 2006; C.A.C. Santos Jr. leg.; MPEG $13348 \bullet$

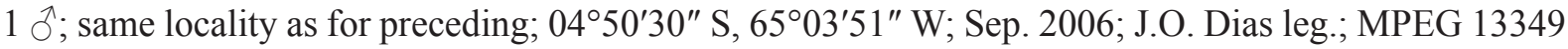
- 2 ठิ $^{\top}$; same locality as for preceding; $04^{\circ} 51^{\prime} 54^{\prime \prime}$ S, $65^{\circ} 20^{\prime} 02^{\prime \prime}$ W; Oct. 2006; N.F. Lo-Man-Hung leg.; MPEG 13352 • 1 ' ; same locality as for preceding; $04^{\circ} 50^{\prime} 32^{\prime \prime}$ S, $65^{\circ} 04^{\prime} 08^{\prime \prime}$ W; Sep. 2006; N.F. Lo-Man-

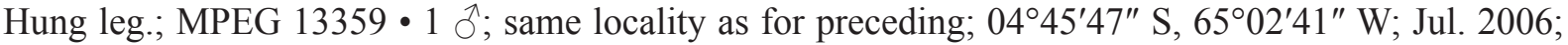
L.T. Miglio leg.; MPEG 13362 • $1 \mathrm{O}^{\text {'; }}$; same locality as for preceding; $04^{\circ} 50^{\prime} 50^{\prime \prime} \mathrm{S}, 65^{\circ} 05^{\prime} 03^{\prime \prime} \mathrm{W}$; Sep. 2006; C.A.C. Santos Jr. leg.; MPEG $13368 \bullet 1$ क ; same locality as for preceding; $04^{\circ} 52^{\prime} 36^{\prime \prime}$ S, $65^{\circ} 09^{\prime} 05^{\prime \prime}$ W; 13 Jul. 2003; J.O. Dias leg.; MPEG 22822 • 1 q; same locality as for preceding; $04^{\circ} 51^{\prime} 17^{\prime \prime} \mathrm{S}, 65^{\circ} 04^{\prime} 14^{\prime \prime} \mathrm{W}$; 17 Jul. 2003; A.B. Bonaldo leg.; MPEG 22830 • 1 đో; same locality as for preceding; 19 Jul. 2002; A.B. Bonaldo leg.; MPEG 22835 • 2 q $\%$; same collection data as for preceding; 21 Jul 2003; J.O. Dias leg.; MPEG 22840 • 1 §̂; ; same locality as for preceding; A.B. Bonaldo leg.; MPEG 22846.

\section{Description}

Female (holotype, MPEG 13322)

Prosoma. Anterior eye row strongly recurved, ALE slightly larger than AME, posterior eye row procurved with subequal eyes; prosoma knobby, light brown with a thin longitudinal yellow line which widens

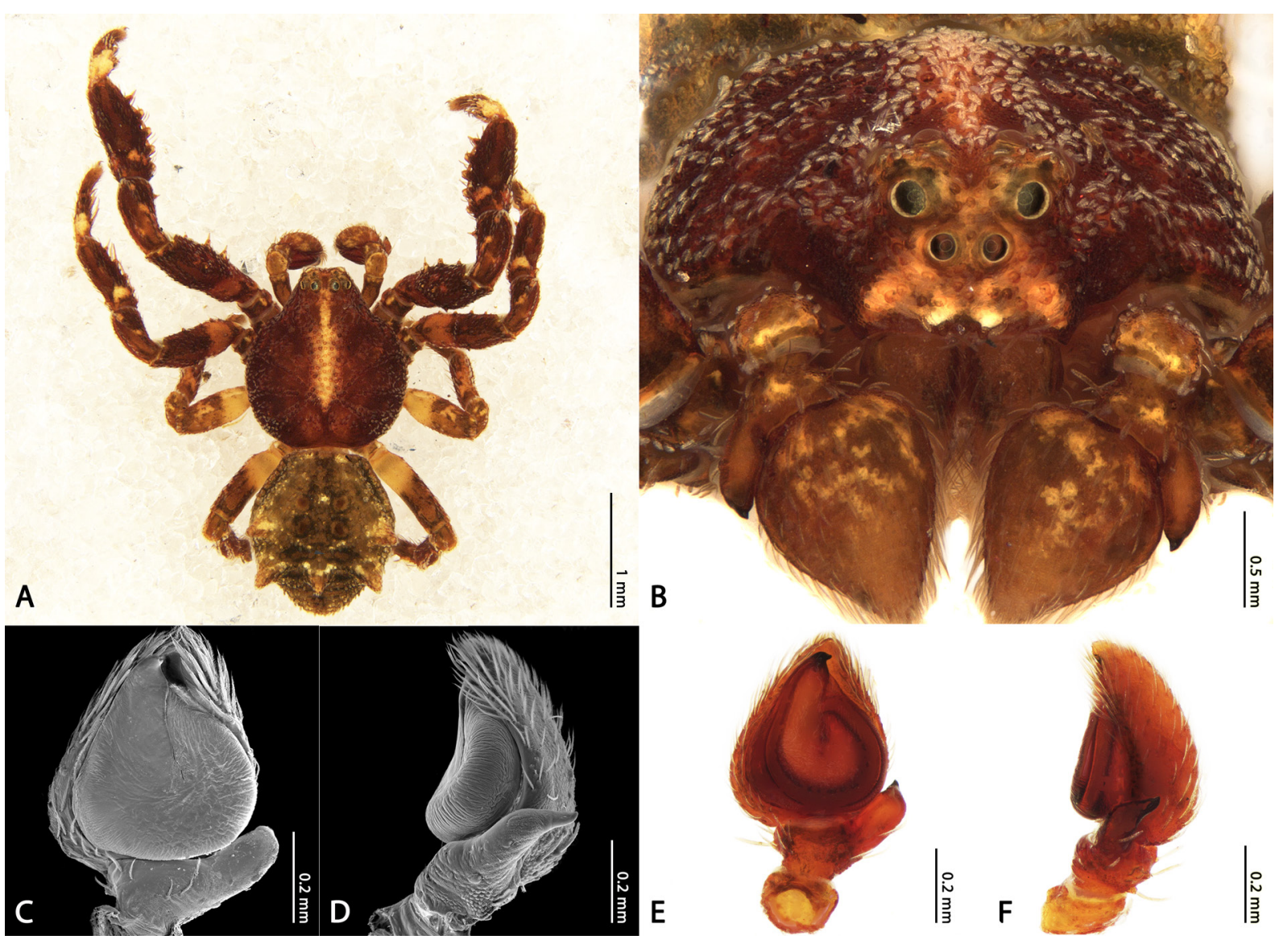

Fig. 5. Male of Kryptochroma hilaris Machado \& Teixeira gen. et sp. nov. (MPEG 13239). A. Habitus, dorsal view. B. Front. C-F. Left male palp. C, E. Ventral view. D, F. Retrolateral view. 
anteriorly on clypeus (Fig. 4A-B). Chelicerae and sternum light brown with yellow stains, endites and labium truncated and totally light brown.

Opisthosoma. Rough surfaced with a large yellow taint covering the entire dorsal region, except the posterolateral abdominal projections.

LEGS. Anterior coxae and trochanters brown with dorsal yellow stains; other segments of legs I and II light brown with darker taints randomly distributed; legs III entirely yellow, except by metatarsi and tarsi and legs IV with femora half yellow, half light brown (Fig. 4A)

Measurements. Eye diameters and eye interdistances: AME 0.06, ALE 0.09, PME 0.08, PLE 0.08, AME-AME 0.08, AME-ALE 0.08, PME-PME 0.14, PME-PLE 0.06, MOQ length 0.32, MOQ width 0.32 ; leg formula: $1-2-4-3$ : leg I - femur 2.95 / patella 1.55 / tibiae 2.33 / metatarsus 1.45 / tarsus 0.93 / total 9.21; II - 2.33/ 1.24/ 1.55/ 1.29/0.77/ 7.18; III - 1.55/ 0.77/ 1.24/0.72/ 0.51/4.79; IV - 2.07/ 0.77/ $1.29 / 0.88 / 0.67 / 5.68$. Total body length 5.94; prosoma 2.84 length, 2.69 width; opisthosoma length 3.10; clypeus 0.16 height; sternum 1.0 length, 0.88 width; endites 0.48 length, 0.29 width; labium 0.32 length, 0.38 width.

\section{Male (MPEG 13239)}

Prosoma. Reddish-brown with a median yellow stripe and dark posterior slope (Fig. 5A-B); MS absent and eye disposition as in females. Sternum scutiform, as long as wide, light brown with a large yellow taint; labium and endites totally light brown and truncated.

LEGS. Legs I and II predominantly light brown with proximal yellow stains on tibiae and distal on metatarsi; posterior legs light brown, except by the bicolor femora (Fig. 5A).

Opisthosoma. Greyish-brown with dark-yellow projections.

MeAsurements. Eye diameters and eyes interdistances: AME 0.06, ALE 0.09, PME 0.06, PLE 0.06, AME-AME 0.06, AME-ALE 0.06, PME-PME 0.11, PME-PLE 0.04, MOQ length 0.24, MOQ width 0.25 ; leg formula: $1-2-4-3$ : leg I - femur 1.32 / patella 0.67 / tibiae 1.03 / metatarsus 0.72 / tarsus 0.45 / total 4.19; II - 0.96/ 0.53/ 0.77/ 0.56/ 0.40/ 3.22; III - 0.72/ 0.40/ 0.64/ 0.33/ 0.32/ 2.41; IV - 0.88/ 0.40/ $0.69 / 0.45 / 0.37 / 2.79$. Total body length 3.06; prosoma 1.61 length, 1.43 width; opisthosoma length 1.45; clypeus 0.16 height; sternum 0.72 length, 0.62 width; endites 0.32 length, 0.19 width; labium 0.16 length, 0.24 width.

\section{Distribution}

Brazil (Amazonas) (Fig. 9).

Kryptochroma macrostyla (Mello-Leitão, 1929) comb. nov. Figs 6, 9, 20C

Stephanopis macrostyla Mello-Leitão, 1929: 61, fig. 145.

\section{Diagnosis}

The male of $K$. macrostyla resembles that of $K$. pentacantha by its robust body and large size when compared to other males of the genus, and by the stout and conical RTA (Figs 6D, F, 20C), but can be easily distinguished by their long, free and filiform embolus (Fig. 6C, E). 


\section{Material examined}

\section{Holotype}

BRAZIL• ○’; Rio de Janeiro, Teresópolis; C.F. Mello-Leitão leg.; MNHN 11467.

\section{Additional material}

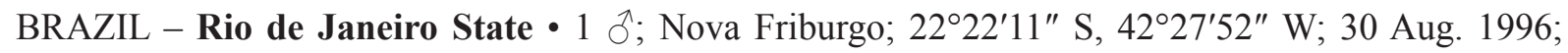

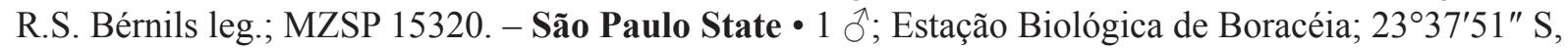

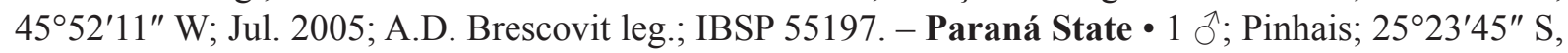
$48^{\circ} 55^{\prime} 58^{\prime \prime} \mathrm{W}$; 15-20 Oct. 1995; lab staff leg.; MCTP 7665.

\section{Description}

Male (MZSP 15320)

Prosoma. Anterior eye row strongly recurved, ALE slightly larger than AME (Fig. 6B); posterior eye row in a straight line with equal sized eyes. Carapace predominantly dark-brown with a longitudinal yellow line that goes from the middle of PME to the edge of the MS (Fig. 6A); clypeus region and ocular tubercles lighter than the rest of the prosoma (Fig. 6B).

LEGS. Legs I and II reddish-brown, coxae I and II dark-brown with yellowish stains; legs III and IV darkbrown except by the femora which are yellow on its proximal region.
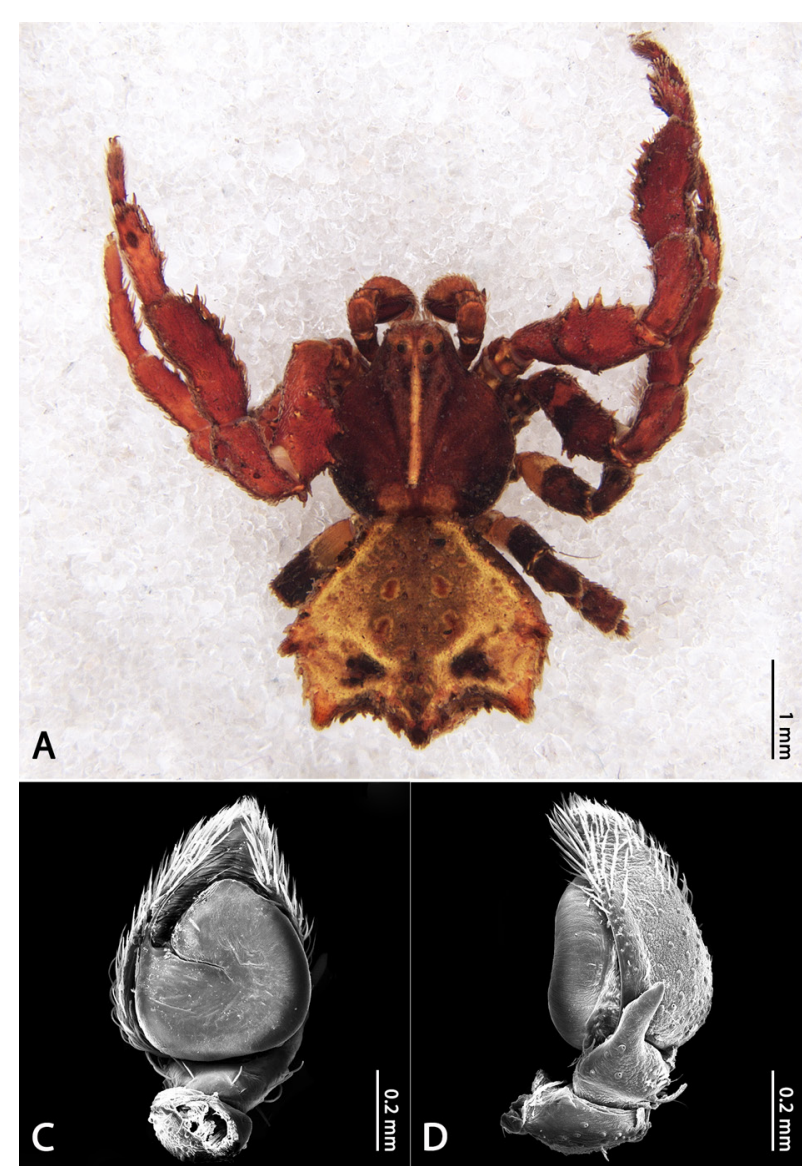
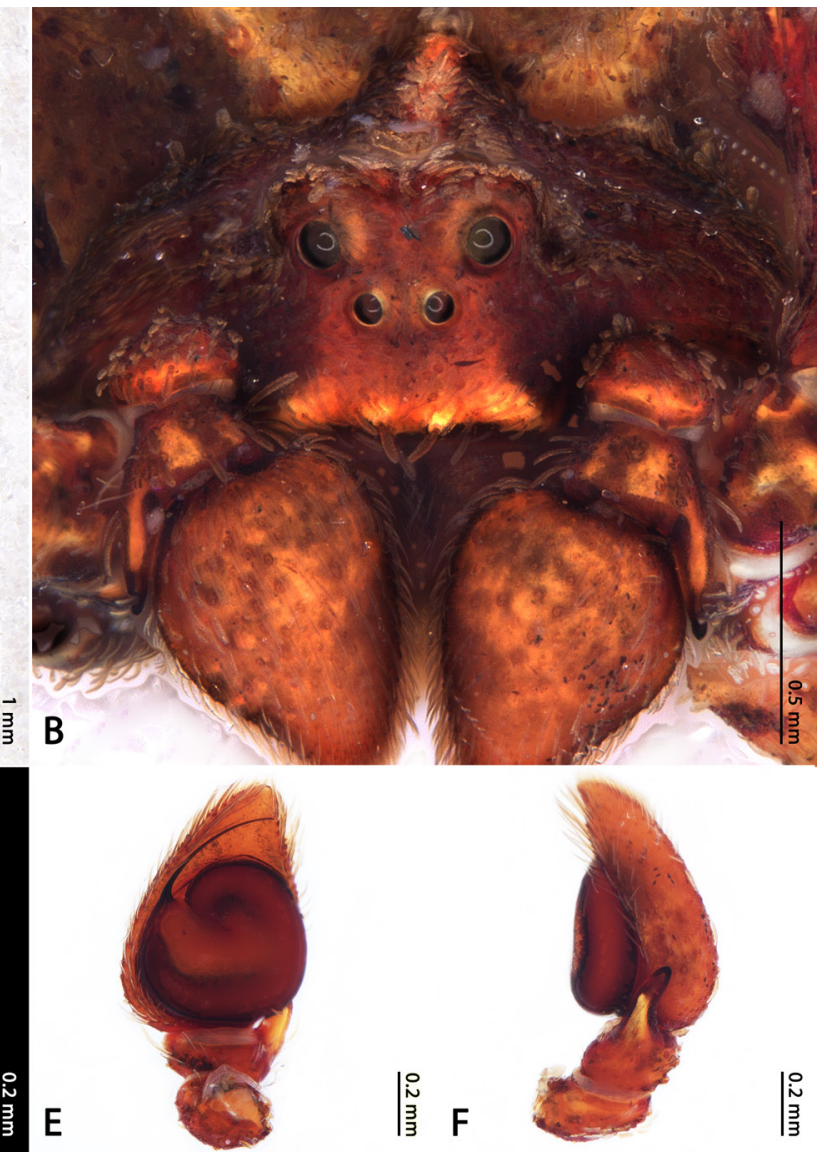

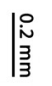

Fig. 6. Male of Kryptochroma macrostyla (Mello-Leitão, 1929) gen. et comb. nov. (MZSP 15320). A. Habitus, dorsal view. B. Front. C-F. Left male palp. C, E. Ventral view. D, F. Retrolateral view. 
Opisthosoma. Rough surfaced, predominantly yellow with dark-brown stains between the abdominal projections and on the sides; lateral projections orange and median dark-brown (Fig. 6A).

MeAsurements. Eye diameters and eye interdistances: AME 0.06, ALE 0.11, PME 0.08, PLE 0.08, AME-AME 0.09, AME-ALE 0.11, PME-PME 0.17, PME-PLE 0.08, MOQ length 0.40, MOQ width 0.38 ; leg formula: $1-2-4-3$ : leg I - femur 2.43 / patella 1.04 / tibiae $1.61 /$ metatarsus 0.96 / tarsus 0.72 / total 6.76; II - 2.22/ 0.88/ 1.25/ 0.88/ 0.59/ 5.82; III - 1.12/ 0.64/ 1.01/ 0.56/ 0.48/ 3.81; IV - 1.29/ 0.61/ $0.96 / 0.64 / 0.53 / 4.03$. Total body length 5.28 ; prosoma 2.59 length, 2.43 width; opisthosoma length 2.69; clypeus 0.27 height; sternum 0.96 length, 0.88 width; endites 0.43 length, 0.24 width; labium 0.27 length, 0.32 width.

\section{Female}

Unknown.

\section{Distribution}

Brazil (Paraná, Rio de Janeiro and São Paulo) (Fig. 9).

Kryptochroma parahybana (Mello-Leitão, 1929) gen. et comb. nov.

Figs 7-9, 20D, 21C

Stephanopis parahybana Mello-Leitão, 1929: 58, fig. 140.

Stephanopis salobrensis - Mello-Leitão 1929: 57, figs 137-139 (misidentification, only female paralectotypes).

\section{Note}

The type series of $S$. salobrensis is constituted by four syntype individuals of two different species. As the male has remarkable genitalic features and clear diagnostic somatic characters (Fig. 26A-B), we designate it as lectotype (see the section Additional taxonomic acts). The rest of the specimens in the type series are a juvenile and two adult females of K. parahybana, misidentified (Fig. 26C-D).

\section{Diagnosis}

The female of K. parahybana resembles that of $K$. hilaris gen. et sp. nov. by the light median line on prosoma and whitish clypeus (Fig. 7A); however, it can be distinguished from this latter by the five pairs of ventral macrosetae on the anterior tibiae instead of four, small and elliptical copulatory openings (Figs 7C, E, 21C) and epigynal plate lacking the median septum. Males may present a body coloration pattern similar to that of K. hilaris gen. et sp. nov.; however, can be easily differentiated by the shorter abdominal projections (Fig. 8A-B), reduced RTA with distal indentations (Figs 8D, F, 20D) and by the apical and sinuous embolus (Figs 8C, E, 20D).

\section{Material examined}

\section{Holotype}

BRAZIL• ○̊; Paraíba; C.F. Mello-Leitão leg.; MNHN 17824.

\section{Paralectotypes of Stephanopis salobrensis}

BRAZIL • 2 q ; Bahia, Salobro; C.F. Mello-Leitão leg.; MNHN 3973

\section{Additional material}

BRAZIL - Amazonas State • 1 \%; Manaus, Reserva da Campina; 2³6'19" S, 6002'11" W; 18 Jul. 1973; L. Albuquerque leg.; INPA • 1 ơ; same collection data as for preceding; 11 Jun. 1973; INPA 


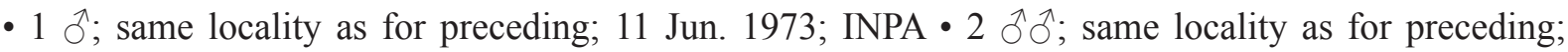
21 Mar. 1974; INPA • 1 क ; same locality as for preceding; 18 Jun. 1973; INPA 1 o ; same locality as for preceding; 18 Jun. 1973; INPA -1 O, 3 juvs; same locality as for preceding; 12 Jan. 1973; INPA • 2 $\hat{\delta}, 1$ juv.; same locality as for preceding; 25 Apr. 1973; INPA • 1 त; Manaus, Reserva

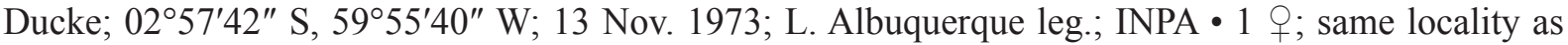
for preceding; INPA $\bullet 1 \hat{\delta}$; same locality as for preceding; 16 Oct. 1973; INPA $\bullet 1$; same locality as

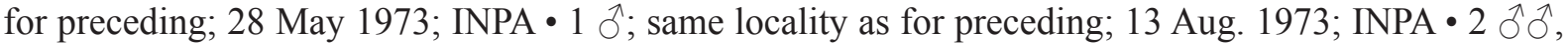
1 juv., same locality as for preceding; 12 Mar. 1987; A.A. Lise leg.; INPA • 1 ô, 2 juvs; same locality as for preceding; 4 Aug. 1987; INPA • 1 \%; same locality as for preceding; 5 Aug. 1987; INPA • 1 \%; same locality as for preceding; 12 Mar. 1987; L. Aquino leg.; INPA • 1 क; Manaus, Fazenda Esteio; $02^{\circ} 22^{\prime} 60^{\prime \prime} \mathrm{S}, 59^{\circ} 51^{\prime} 00^{\prime \prime} \mathrm{W}$; 30 Oct. 1985; B.C. Klein leg.; INPA $1208 \bullet 1$ '̊; Manaus, PDBFF Dimona; $02^{\circ} 25^{\prime} 00^{\prime \prime} \mathrm{S}, 60^{\circ} 00^{\prime} 00^{\prime \prime} \mathrm{W}$; 20 Dec. 2001-20 Mar. 2002; F. Rego \& A. Cardoso leg.; INPA 2772 • 1 ○; same locality as for preceding; INPA $2773 \bullet 1 \delta^{\lambda}$; same locality as for preceding; INPA $2774 \bullet 1 \delta^{\lambda}$; same locality as for preceding; INPA $2775 \bullet 1$; same locality as for preceding, INPA $2776 \bullet 1$; ; Manaus,

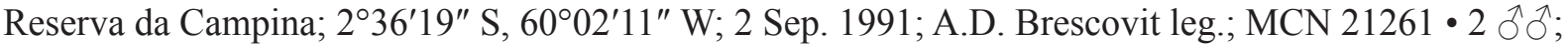
Manaus, Reserva Ducke; 02 57'42" S, 5955'40" W; 14-25 Aug. 1991; A.D. Brescovit leg.; MCTP

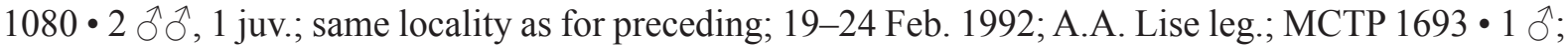
same locality as for preceding; 4 Aug. 1987; MCTP 29942 - 1 ô, 3 juvs; same locality as for preceding; 12 Mar. 1987; MCTP 29943 • 1 क; same locality as for preceding; 28 May 1973; L. Albuquerque leg.; MCTP 29944 • 1 ô, 1 ㅇ, 1 juv.; Manaus, Reserva da Campina; 2³6'19" S, 6002'11" W; 25 Apr. 1973; L. Albuquerque leg.; MCTP 29946 • 1 đo, 1 q, 1 juv.; same locality as for preceding; 12 Jan. 1973; MCTP 29947 • 1 क; same locality as for preceding; 14 May 1973; MCTP $29948 \bullet 1$ q; Manaus, Br. 174.

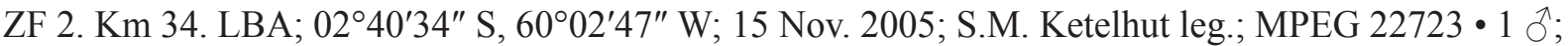

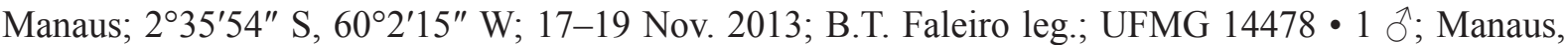
Reserva Ducke; 02 57'42" S, 5955'40" W; 14-25 Aug. 1991; A.D. Brescovit leg.; MACN-Ar 39669 - 1 đ̃; same locality as for preceding; 12 Mar. 1987; A.A. Lise leg.; MACN-Ar 39672. - Pará State •

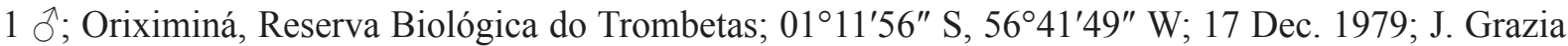

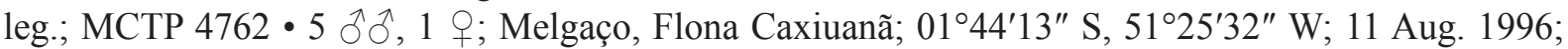

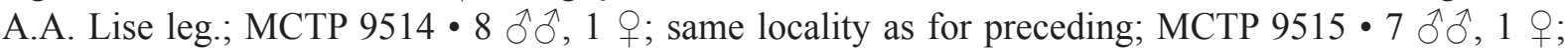

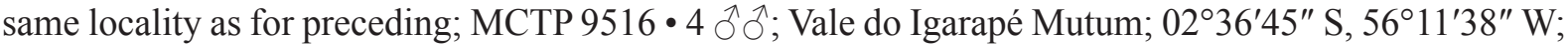
4 Aug. 2004; D.R. Santos-Souza leg.; MPEG 8767 • 2 đơ; Juruti, Sítio Três Irmãos; 02 $27^{\circ} 51^{\prime \prime}$ S, 5600'08" W; 4 Mar. 2006; S.C. Dias leg.; MPEG 9067 • 1 ô; Melgaço, Estação Científica Ferreira

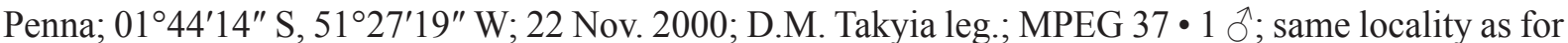

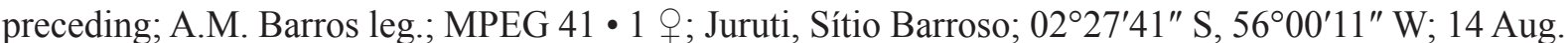

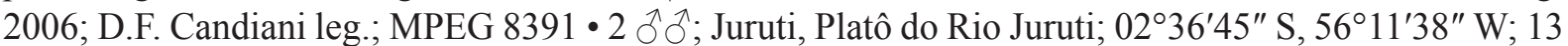

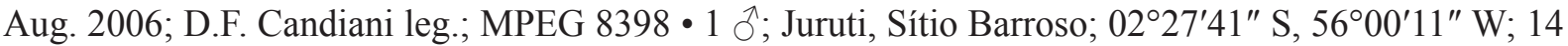
Aug. 2006; N.F. Lo-Man-Hung leg.; MPEG 8415 • 1 万ै; same locality as for preceding; MPEG $8430 \bullet$

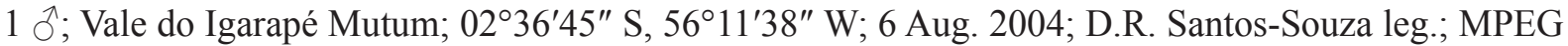

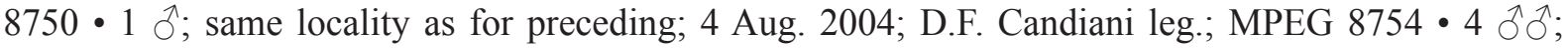

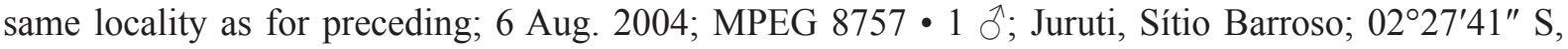
56 $00^{\circ} 11^{\prime \prime}$ W; 13 Aug. 2004; D.R. Santos-Souza leg.; MPEG 8758 • 1 ㅇ; same locality as for preceding; MPEG 8762 • 1 q; Vale do Igarapé Mutum; 02³6'45" S, 56¹1'38" W; 5 Aug. 2004; D.F. Candiani leg.;

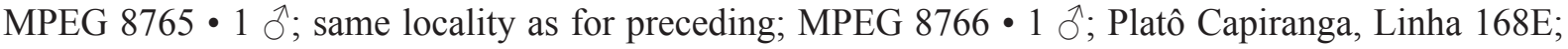
$02^{\circ} 28^{\prime} 22^{\prime \prime}$ S, 56 $16^{\circ} 29^{\prime \prime}$ W; 10 Mar. 2006; D.R. Santos-Souza leg.; MPEG $9062 \bullet 3$ đô; same locality as

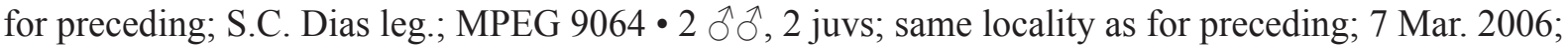

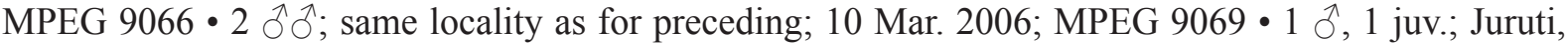

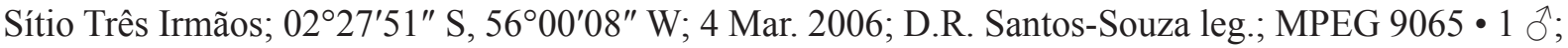
same locality as for preceding; $02^{\circ} 27^{\prime} 41^{\prime \prime} \mathrm{S}, 56^{\circ} 00^{\prime} 11^{\prime \prime} \mathrm{W}$; 11 Feb. 2007; J.A.P. Barreiros leg.; MPEG 14941 • 1 q; same locality as for preceding; 8 Feb. 2007; N.F. Lo-Man-Hung leg.; MPEG $14944 \bullet 1$ 万; same locality as for preceding; $02^{\circ} 36^{\prime} 45^{\prime \prime}$ S, 56 $6^{\circ} 11^{\prime} 38^{\prime \prime}$ W; 7 Feb. 2007; J.A.P. Barreiros leg.; MPEG 


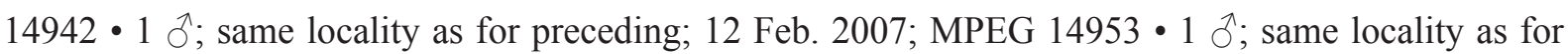
preceding; 02 $27^{\prime} 51^{\prime \prime}$ S, 56 $56^{\circ} 00^{\prime} 08^{\prime \prime}$ W; 11 Feb. 2007; J.A.P. Barreiros leg.; MPEG 14951 • 1 O; Melgaço;

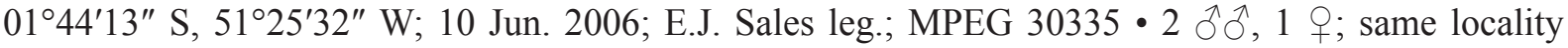
as for preceding; J.A.P. Barreiros leg.; MPEG 30336 • 1 q; same locality as for preceding; 27 May 2006; E.J. Sales leg.; MPEG 30337 • 1 क; same locality as for preceding; 31 May 2006; B.B. Santos leg. MPEG 30338 • 1 o ; same locality as for preceding; 5 Jun. 2006; B.C. Araújo leg.; MPEG 30339 - 1 O , 1 क; same locality as for preceding; 26 May 2006; E.J. Sales leg.; MPEG $30340 \bullet 1$ ㅇ; same

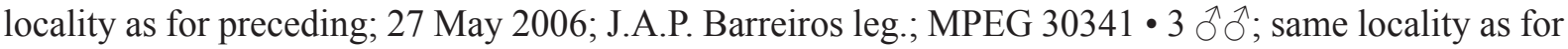
preceding; 5 Jun. 2006; B.C. Araújo leg.; MPEG $30342 \bullet 4 \hat{\jmath} \widehat{\jmath}$; same locality as for preceding; 1 Jun. 2006; B.B. Santos leg.; MPEG 30343 • 1 đ̂; same locality as for preceding; 26 May 2006; E.J. Sales leg.; MPEG $30344 \bullet 6$ ô; same locality as preceding; 1 Jun. 2006; J.A.P. Barreiros leg.; MPEG $30345 \bullet$

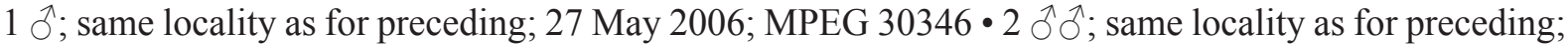
5 Jun. 2006; B.C. Araújo leg.; MPEG 30347 • 1 \%; Juruti, Sítio Três Irmãos; $02^{\circ} 27^{\prime} 51^{\prime \prime}$ S, 56 $06^{\circ} 00^{\prime \prime}$ W;

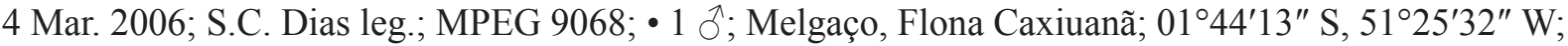
11 Aug. 1996; A.A. Lise leg.; MACN-Ar 39670 1 गे; same locality as for preceding; MACN-Ar 39671.

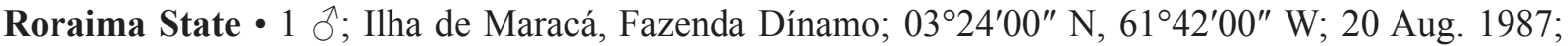
A.A. Lise leg.; MCTP 29949.

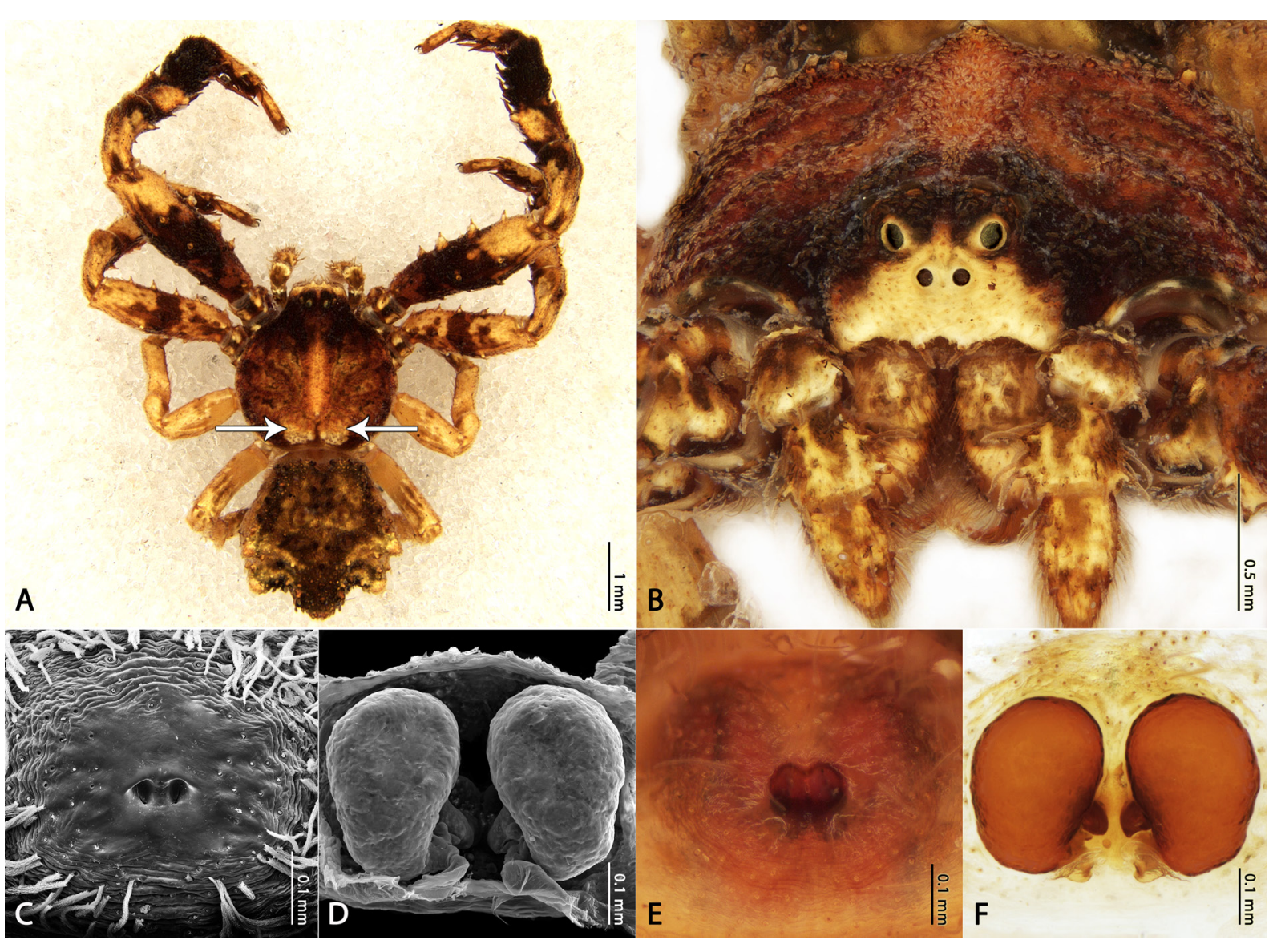

Fig. 7. Female of Kryptochroma parahybana (Mello-Leitão, 1929) gen. et comb. nov. (MPEG 30339) A. Habitus, dorsal view (arrows indicate the pair of circular taints on the posterior slope of the prosoma). B. Front. C, E. Epigynal plate, ventral view. D, F. Spermathecae, dorsal view. 
FRENCH GUIANA • 1 万’; Sant-Élie, Trinité Reserve; $04^{\circ} 35^{\prime} 00^{\prime \prime}$ N, $53^{\circ} 18^{\prime} 00^{\prime \prime}$ W; 7 Oct. 2010; V. Vedel

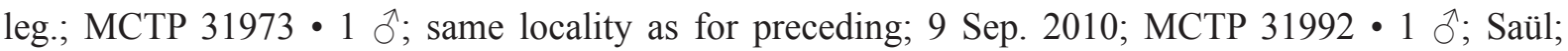
$3^{\circ} 37^{\prime 2} 22^{\prime \prime}$ N, 5312'30" W; 2015; V. Vedel leg.; MCTP 41965 • 1 ô; same locality as for preceding; MCTP $41966 \bullet 1$; ; same locality as for preceding; MCTP $41967 \bullet 1$; ; same locality as for preceding;

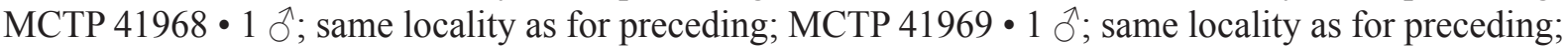

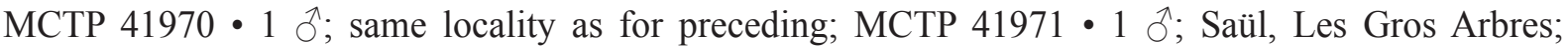
03³7'29" N, 53¹2'28" W; 31 Mar. 2011; D. Comus leg.; MCTP 31965.

\section{Description}

Female (MPEG 30339)

Prosoma. Anterior eye row strongly recurved, posterior eye row slightly procurved, ocular mounds discrete (Fig. 7A-B). Carapace predominantly reddish-brown with black pigmentation increasing on the sides and on the cephalic area; pair of posterior slope scars whitish-yellow and surrounded by a pair of setae emerging from cylindrical sockets (Fig. 7A-B). Chelicerae yellow with dark-brown taints, sternum slightly longer than wide, brown with a median yellow taint; endites and labium truncated, brown.

Legs. Legs I and II present femora and tibiae predominantly black, with some yellow regions, while posterior legs are lighter, predominantly light-yellow with few light brown spots (Fig. 7A).
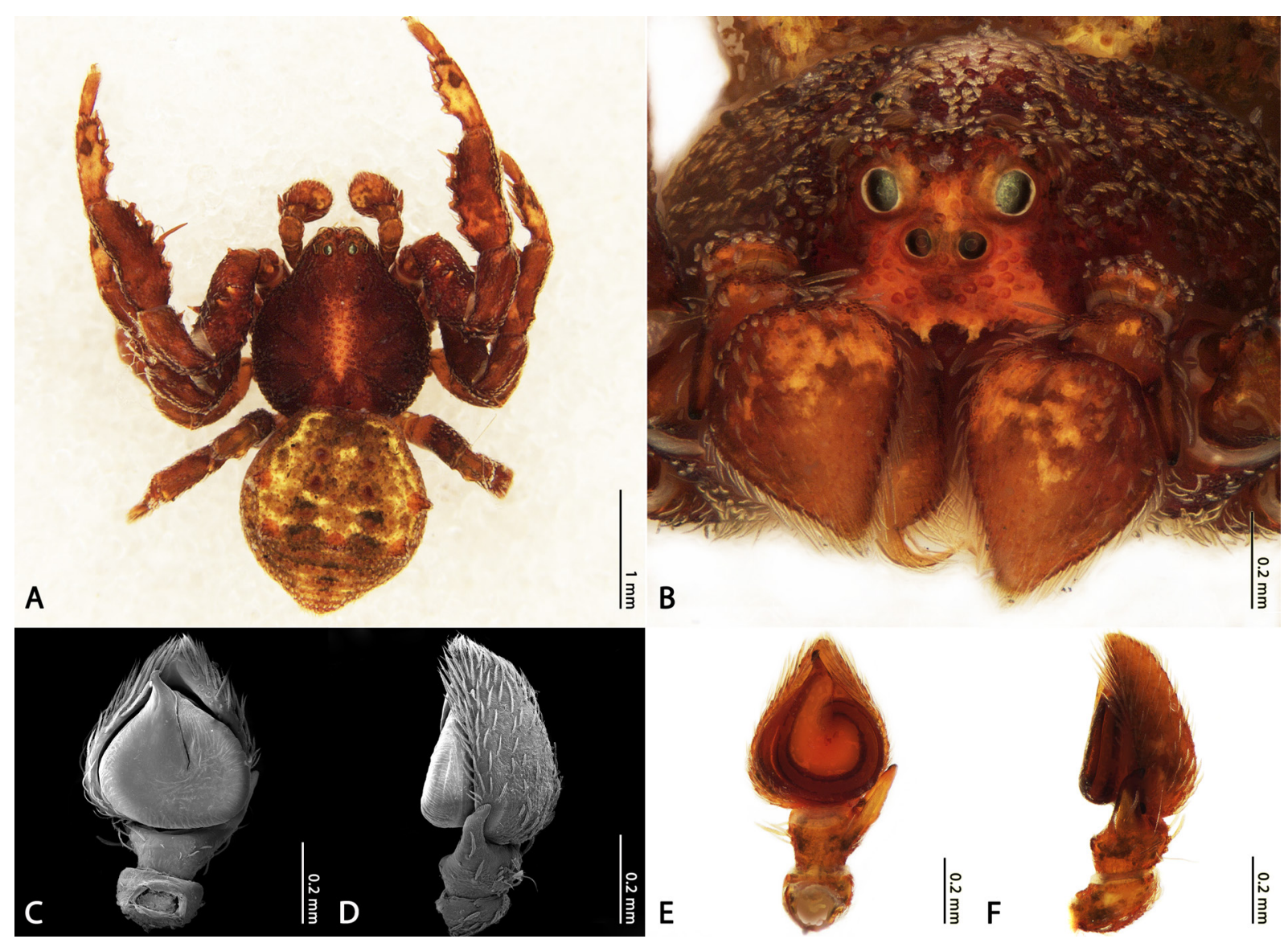

Fig. 8. Male of Kryptochroma parahybana (Mello-Leitão, 1929) gen. et comb. nov. (MPEG 9069). A. Habitus, dorsal view. B. Front. C-F. Left male palp. C, E. Ventral view. D, F. Retrolateral view. 
Opisthosoma. Opisthosoma predominantly brown with yellow pigmentation on the edge of the abdominal projections; spermathecae rounded, slightly narrowed posteriorly (Fig. 7D, F).

MEAsurements. Eye diameters and eye interdistances: AME 0.06, ALE 0.13, PME 0.09, PLE 0.09, AMEAME 0.09, AME-ALE 0.11, PME-PME 0.22, PME-PLE 0.04, MOQ length 0.38, MOQ width 0.43; leg formula: 1-2-4-3: leg I - femur 3.62/ patella 2.17/ tibiae 2.84/ metatarsus 1.76 / tarsus 1.03 / total 11.42; II 2.95/ 1.65/2.12/ 1.34/ 0.93/ 8.99; III - 1.76/ 1.03/ 1.60/ 0.77/ 0.56/5.72; IV - 1.96/ 1.03/ 1.55/ 1.03/ 0.72/ 6.29. Total body length 7.24; carapace 3.62 length, 3.36 width; opisthosoma length 3.62 ; clypeus height 0.24; sternum 1.20 length, 1.04 width; endites 0.61 length, 0.33 width; labium 0.40 length, 0.45 width.

\section{Male (MPEG.ARA 9069)}

Prosoma. Anterior eye row strongly recurved, posterior slightly procurved; prosoma predominantly brownish-red with median thoracic region and clypeus light-yellow (Fig. 8A-B). Labium, endites and chelicerae brown.

LEGS. Predominantly brownish-red with yellowish taints on anterior tarsi (I and II) and on the proximal half of the posterior femora (III and IV).

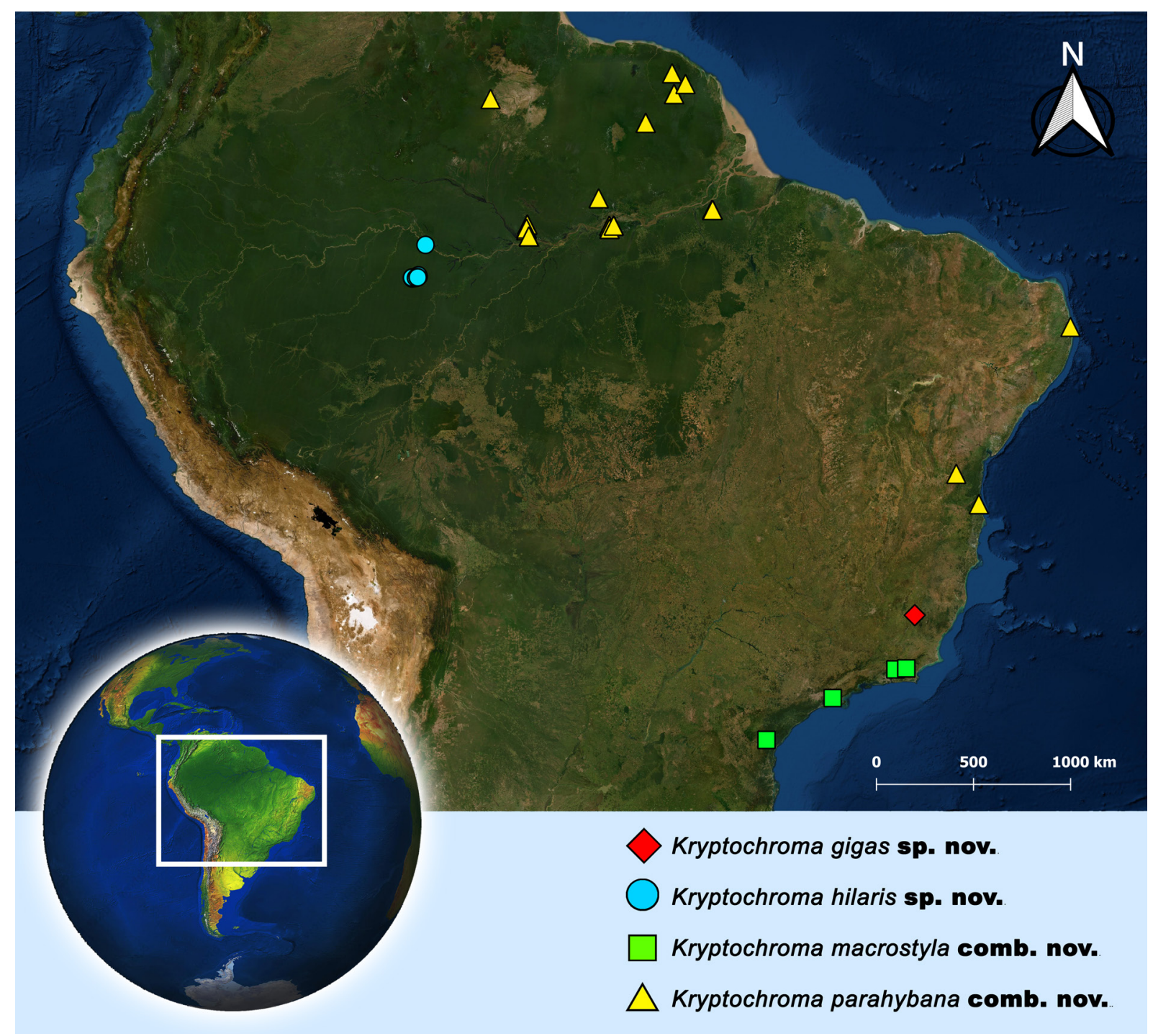

Fig. 9. Distribution records of species of Kryptochroma Machado gen. nov. 
Opisthosoma. Predominantly yellow with a whitish line-like macula connecting the five reddish spiniform projections (Fig. 8A).

MeAsurements. Eye diameters and eye interdistances: AME 0.08, ALE 0.12, PME 0.10, PLE 0.10, AME-AME 0.06, AME-ALE 0.06, PME-PME 0.16, PME-PLE 0.06, MOQ length 0.44, MOQ width 0.30 ; leg formula: $1-2-4-3$ : leg I - femur 1.60 / patella 0.90 / tibiae 1.10 / metatarsus 0.90 / tarsus 0.50 / total 5 ; II - 1.40/ 0.76/ 1/ 0.70/0.44/ 4.30; III - 0.90/ 0.50/ 0.80/ 0.50/ 0.40/3.10; IV - 1.10/ 0.50/ 0.80/ 0.56/ $0.40 / 3.36$. Total body length 4.03; carapace 2 length, 1.76 width; opisthosoma length 2.03; clypeus height 0.20 ; sternum 0.90 length, 0.84 width; endites 0.48 length, 0.24 width; labium 0.26 length, 0.26 width.

\section{Distribution}

Brazil (Amazonas, Bahia, Pará, Roraima) and French Guiana (Maripasoula, Saint-Élie and Saül) (Fig. 9).

Kryptochroma pentacantha (Mello-Leitão, 1929) gen. et comb. nov.

Figs 1, 10-11, 18, 19A, 22A

Stephanopis pentacantha Mello-Leitão, 1929: 59.

Stephanopis bellus Soares \& Soares, 1946: 58, fig. 7. syn. nov.

Stephanopis colatinae Soares \& Soares, 1946: 60, figs 8-9. syn. nov.

Stephanopis quinquetuberculata (misidentification) - Machado et al. 2017: 454, figs s4d, s5b, s7e, s10c, s11d, s13e, s14d.

\section{Diagnosis}

The male of $K$. pentacantha resembles that of $K$. quadrata sp. nov. by the shape of the tegulum and embolus (Fig. 11C, E); however, it can be distinguished by its stout and conical RTA (Figs 11D, F, 19A) and larger body size, since they are the largest males of genus, being the sexual size dimorphism in this species less pronounced. Females can be recognized by the external genitalia bearing a noticeable median septum and a pair of long longitudinal depressions leading to the copulatory openings (Figs 10C, E, 22A). The most common body color pattern for both males and females usually present a diagnostic median white spot on the dorsum of the opisthosoma (Figs 10A, 11A).

\section{Material examined}

Lectotype (designated here)

BRAZIL • ${ }^{\text {; }}$; Minas Gerais, Caraça; C.F. Mello-Leitão leg.; MNHN 8227.

Paralectotypes (designated here)

BRAZIL・ 2 우 $0,3 \hat{\jmath} \widehat{\jmath}$; Rio de Janeiro; C.F. Mello-Leitão leg.; MNHN 8203.

Holotype of $S$. bellus

BRAZIL • + ; Espírito Santo, Colatina, Rio São José; 29 Sep. 1942; B.A.M Soares leg.; MZSP 661.

\section{Holotype of $S$. colatinae}

BRAZIL• ○`; Espírito Santo, Colatina, Rio São José; 21 Sep. 1942; B.A.M Soares leg.; MZSP 566.

\section{Additional material}

BRAZIL - Amazonas State - 1 O’; Manaus, Fazenda Esteio; 02 ${ }^{\circ} 22^{\prime} 60^{\prime \prime}$ S, 59 51'00" W; 16 Dec. 1988; M.V.B. Garcia leg.; SMNK • 1 ग̊; São Gabriel da Cachoeira, Morro dos Seis Lagos; 0 16'57" N, 6640'44" W; 1 Oct. 1990; A.A. Lise leg.; MCTP 1195. Espírito Santo State • 1 q; São Mateus, 
Reserva Florestal Vale do Rio Doce; $19^{\circ} 09^{\prime} 05^{\prime \prime}$ S, 4004'15" W; 19-25 Jul. 1997; A.D. Brescovit et al. leg.; IBSP $12719 \cdot 3 \hat{\jmath}$; ; same locality as for preceding; IBSP $24354 \cdot 1$ ô; Santa Tereza, Estação Biológica Santa Lucia; 1957'54" S, 40³2'25" W; May 2007; T. Souza et al. leg.; IBSP 160118 • 1 ð’; same locality as for preceding; IBSP 160128 • 1 ô, 1 o ; Linhares; $19^{\circ} 23^{\prime} 27^{\prime \prime} \mathrm{S}, 40^{\circ} 04^{\prime} 19^{\prime \prime} \mathrm{W}$; Aug. 1997; A. Santos leg.; MCTP 10366 • 1 o $^{\lambda}$; same locality as for preceding; $19^{\circ} 57^{\prime} 43^{\prime \prime}$ S, 40³1'56" W; 7 Sep. 1999; R.L.C. Baptista leg.; MNRJ 13922 • 1 त; same locality as for preceding; 7 Jul. 2013; P.H. Martins and M.T.T. Santos leg.; UFMG 14048 • $10^{\text {`}}$; Domingos Martins; $20^{\circ} 25^{\prime} 57^{\prime \prime} \mathrm{S}, 41^{\circ} 01^{\prime} 12^{\prime \prime} \mathrm{W}$;

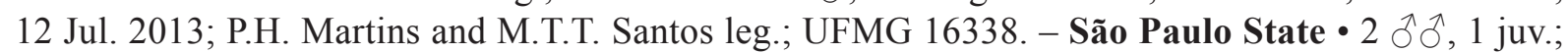
Itanhaém, Ilha da Queimada Grande; $2^{\circ} 29^{\prime} 16^{\prime \prime} \mathrm{S}, 46^{\circ} 40^{\prime} 27^{\prime \prime} \mathrm{W}$; 4-5 Dec. 1995; A.D. Brescovit and

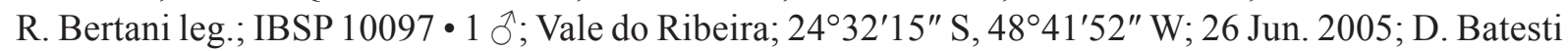
leg.; IBSP 55502 • 1 ऊ ; Guarapiranga, Parque Ilha dos Eucaliptos; $23^{\circ} 44^{\prime} 02^{\prime \prime} \mathrm{S}, 46^{\circ} 44^{\prime} 00^{\prime \prime} \mathrm{W}$; 6-12

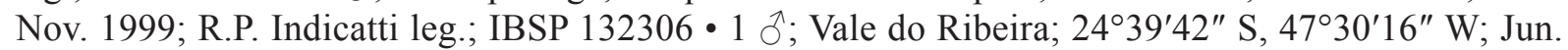

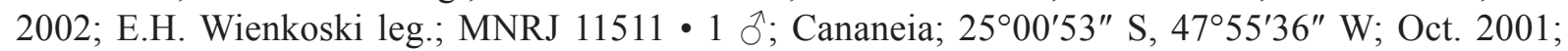

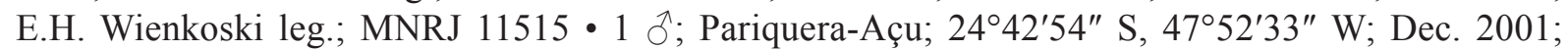

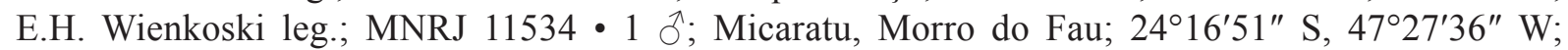
19 Dec. 1998; R. Pinto-da-Rocha, R.S. Bérnils and E. Howaldt leg.; MZSP 16980 • 1 q; Peruíbe,

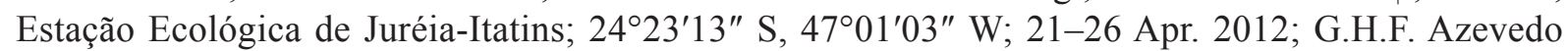
and J.P.P. Pena-Barbosa leg.; UFMG 13029. - Paraná State - 1 ô; Morretes, Serra da Graciosa; 2522'31" S, 48 52'09" W; 9-20 Jan. 1995; A.A. Lise et al. leg.; MACN-Ar 39674 • 1 ô, 4 juvs; same collection data as for preceding; lab staff leg.; MCTP $7166 \bullet 1 \hat{\jmath}, 2$ juvs; same locality as for

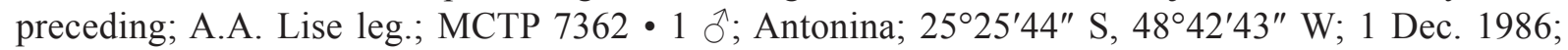

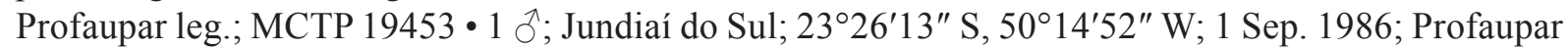
leg.; MCTP 19646 • 1 गै, 2 juvs; same locality as for preceding; $27^{\circ} 17^{\prime} 45^{\prime \prime} \mathrm{S}, 48^{\circ} 21^{\prime} 25^{\prime \prime} \mathrm{W}$; 6 Oct. 1995; A.A. Lise leg.; MCTP $7447 \bullet 1$ o $^{\top}$; same locality as for preceding; $27^{\circ} 17^{\prime} 45^{\prime \prime} \mathrm{S}, 48^{\circ} 21^{\prime} 25^{\prime \prime} \mathrm{W}$; 3 May 1996; A.A. Lise leg.; MCTP 9703. - Santa Catarina State • 1 ơ; São Francisco do Sul, Centro de Estudos e Pesquisas Ambientais Vila da Glória; 26¹3'13" S, 48 41'19" W; 12-15 Dec. 2011; I.L.F. Magalhães leg.; UFMG 10317 • 1 đె’; Reserva Biológica Marina do Arvoredo; $27^{\circ} 17^{\prime} 45^{\prime \prime}$ S,

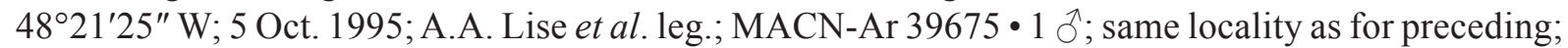
14 Oct. 1994; MACN-Ar 39676 • 1 ' ; same locality as for preceding; $27^{\circ} 17^{\prime} 45^{\prime \prime} \mathrm{S}, 48^{\circ} 21^{\prime} 25^{\prime \prime} \mathrm{W}$;

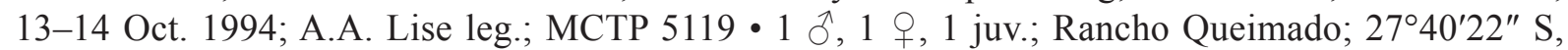
4901'19" W; 8-12 Oct. 1994; A.B. Bonaldo and L.A. Moura leg.; MCTP $5960 \bullet 1$ ठ̊; same locality

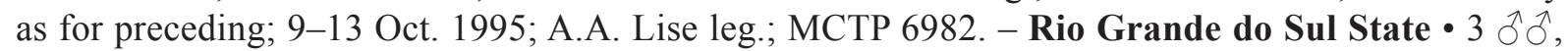
2 웅, 10 juvs; Montenegro; $9^{\circ} 41^{\prime} 20^{\prime \prime}$ S, 51 ${ }^{\circ} 27^{\prime} 39^{\prime \prime}$ W; 3 Nov. 1977; A.A. Lise leg.; MCN 8075 •

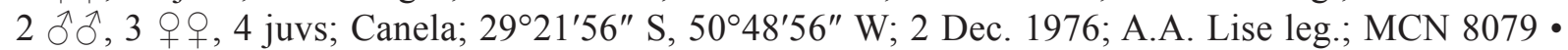

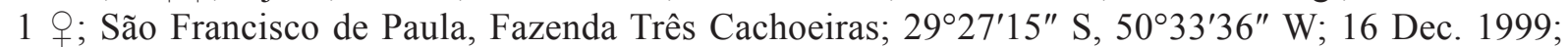

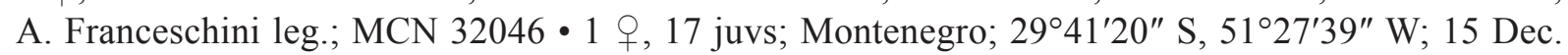
1977; A.A. Lise leg.; MCN 7509 • 1 क , 4 juvs; same locality as for preceding; 29 Sep. 1977; MCN 8080 • 1 O̊, 4 juvs; Maquiné, Estação Experimental da Fepagro; 29³9'41" S, 50¹2'47" W; 6-8 Mar. 1998; L. Moura leg.; MCN 29059 - 3 ổ ô, 1 ๆ, 10 juvs; São Francisco de Paula, Barragem dos Bugres; 29²0'34" S, 5041'46" W; 1-4 Feb. 1999; L. Moura leg.; MCN 30853 • 1 \%; Estrela Velha, Barragem de Itaúba; 2915'36" S, 5314'14" W; 17-21 Jan. 2000; A. Franceschini leg.; MCN 32130 - 1 q; same locality as for preceding; 7 Mar. 2001; R. Ott leg.; MCN 33697 • 1 đ; São Francisco de Paula; 29²6'52" S, 50³5'02" W; 21-24 Mar. 1996; A.A. Lise leg.; MCTP 9855 • 1 q; Santa Maria;

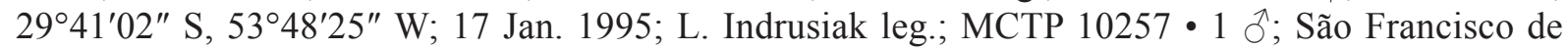
Paula; 29²6'52" S, 50³5'02" W; 9-12 Jan. 1997; A.A. Lise leg.; MCTP 10742 • 1 đ̊; São Francisco de Paula, Potreiro Velho, Pró-Mata; 29²8'51" S, 50¹0'28" W; 5-8 Dec. 1996; A.A. Lise leg.; MCTP

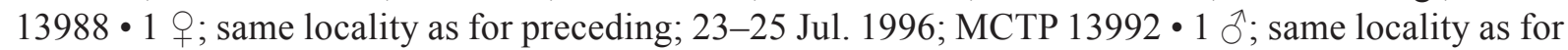
preceding; Dec. 2001; L.A. Bertoncello and A.A. Lise leg.; MCTP $19427 \bullet 1$; ; same locality as for preceding; Sep. 2001; MCTP 25750 • 1 ठ; same locality as for preceding; Oct. 2001; MCTP 25761 - 1 § ; same locality as for preceding; Jul. 2001; MCTP 25762 • 1 क ; same locality as for preceding; 


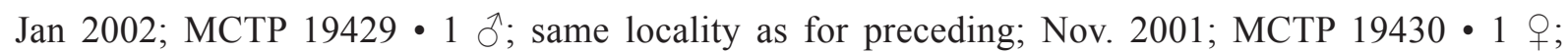
same locality as for preceding; Feb. 2002; MCTP 19435 - 1 q; same locality as for preceding; Dec. 2001; MCTP 19437 • 1 ㅇ; Maquiné, Barra do Ouro; 29³2'18" S, 50¹4'48" W; 23 Mar. 2011; R.A. Teixeira leg.; MCTP 35069 • 1 §̧; same locality as for preceding; 5 Jun. 2011; MCTP $35109 \bullet 1$ O ; same locality as for preceding; 4 May 2011; MCTP 39906 • 1 đ; Floresta Nacional de "São Chico"; 29²5'24" S, 50²3'13.0" W; 19 Dec. 2010; R.A. Teixeira leg.; MCTP 39903 • 1 o; same locality as for preceding; 23 Jan. 2011; MCTP 39904 • 1 क; Viamão; 3004'25" S, 51 $06^{\prime} 27^{\prime \prime}$ W; 23 Sep.

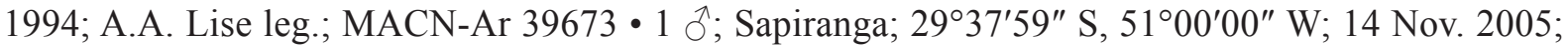
E.L.C. da Silva leg.; MCTP 19755 • 1 क , 1 juv.; Maquiné, Barra do Ouro; 29³2'18" S, 50¹4'48" W; 3 Dec. 2010; R.A. Teixeira leg.; MCTP 31532 • 1 q; Santa Maria, Cidade dos Meninos; 2940'31" S, 53²4'10" W; 15 Jan. 1998; L. Indrusiak and M. Monteiro leg.; MCTP 38671 • 1 ơ; Maquiné, Barra

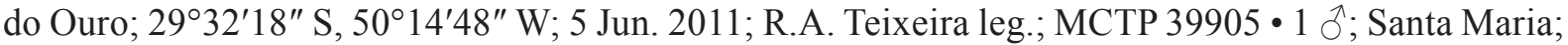

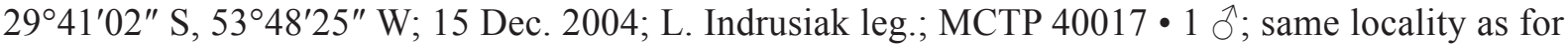
preceding; 27 Aug. 2003; MCTP 40019 • 1 సं; Viamão, Estação Fitotécnica de Viamão; 3002'11" S,

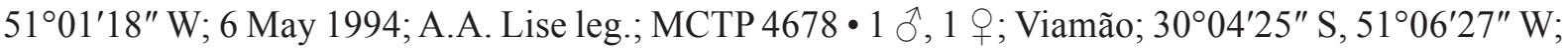
23 Sep. 1994; A.A. Lise leg.; MCTP 8407 • 1 đ’; São Francisco de Paula, Potreiro Velho; 29²8'51" S, $50^{\circ} 10^{\prime} 28^{\prime \prime}$ W; Jul. 2001; L.A. Bertoncello and A.A. Lise leg.; MCTP $19434 \bullet 1$ ô; same locality as for preceding; Oct. 2001; MCTP 19436.

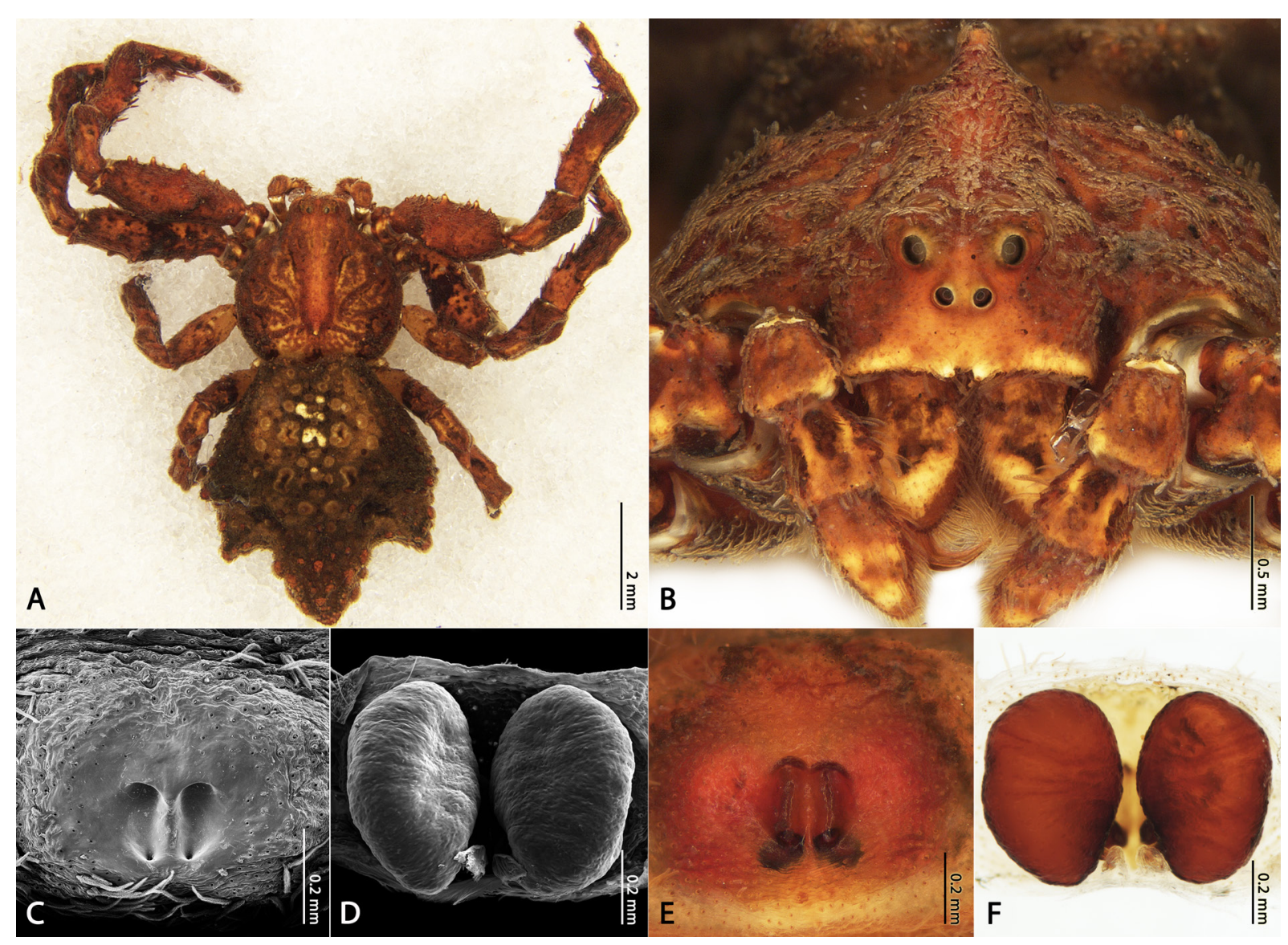

Fig. 10. Female of Kryptochroma pentacantha (Mello-Leitão, 1929) gen. et comb. nov. (MCTP 38671) A. Habitus, dorsal view. B. Front. C, E. Epigynal plate, ventral view, D, F. Spermathecae, dorsal view. 


\section{Description}

Female (MCTP 38671)

Prosoma. Anterior eye row strongly recurved and posterior slightly procurve, carapace predominantly dark-brown with yellowish stains on sides; thoracic region with a pronounced median spire (Fig. 10AB). Clypeus lighter than the rest of carapace, with a marginal yellowish triangle-shaped mark; chelicerae brown with yellowish stains (Fig. 10B), sternum scutiform, slightly longer than wide, dark-brown with a yellow median macula, endites and labium truncated and totally dark-brown.

Legs. Predominantly dark-brown with dispersed lighter stains; femora I and II dorsally enlarged, suffused with dorsolateral and ventral conical sockets with a conical macrosetae in each one; tibia I and II with four pairs of ventral macrosetae (Fig. 10A).

Opisthosoma. Dark-brown, rough surfaced, with concave anterior border and a remarkable white taint between the anterior muscular sigilla; the median posterior opisthosomal projection presents acuminate orange tubercles at the tip, with many leaf-shaped setae (Fig. 10B).

MEAsurements. Eye diameters and eye interdistances: AME 0.09, ALE 0.14, PME 0.09, PLE 0.09, AMEAME 0.09, AME-ALE 0.12, PME-PME 0.24, PME-PLE 0.08, MOQ length 0.45, MOQ width 0.40; leg formula: 1-2-4-3: leg I - femur 3.83/ patella 2.07/ tibiae 2.59/ metatarsus 1.81/ tarsus 1.29/ total 11.59; II 3.78/1.96/2.38/1.76/1.29/11.17; III - 1.96/1.29/1.70/1.03/0.77/6.75; IV-2.48/1.29/1.81/ 1.34/0.82/
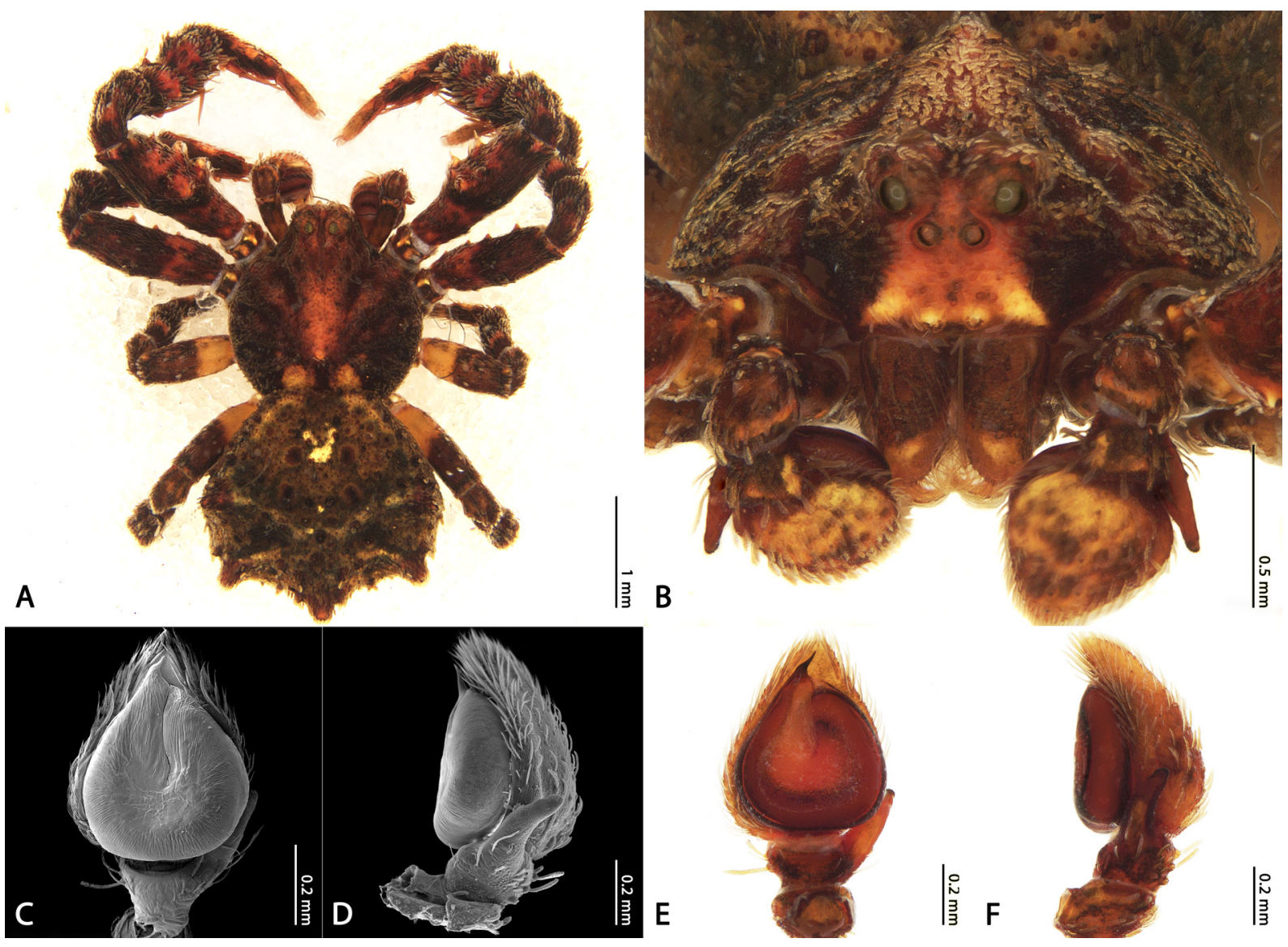

Fig. 11. Male of Kryptochroma pentacantha (Mello-Leitão, 1929) gen. et comb. nov. (MCTP 25762). A. Habitus, dorsal view. B. Front. C-F. Left male palp. C, E. Ventral view. D, F. Retrolateral view. 
7.74. Total body length 9.81; carapace 4.14 length, 3.83 width; opisthosoma length 4.87 ; clypeus 0.28 height; sternum 1.41 length, 1.12 width; endites 0.74 length, 0.38 width; labium 0.41 length, 0.51 width.

Male (MCTP 25762)

Prosoma. Eye disposition and carapace coloration pattern as in female, except by the posterior slope scars, which are yellow (Fig. 11A). Front lighter than the rest of carapace due to a yellowish triangular mark that extends from the clypeus until the ALE (Fig. 11B). Sternum scutiform, as long as wide, darkbrown with a yellow taint; labium and endites totally dark-brown and truncated.

LEGs. Metatarsi of all legs with a distal yellow stain and posterior femora noticeably bicolor, half yellow, half dark-brown; other leg characteristics as in females (Fig. 11A).

Opisthosoma. As in female, except by the shorter median posterior opisthosomal projection and the lack of agglomerated orange tubercles.

MeAsurements. Eye diameters and eye interdistances: AME 0.08, ALE 0.11, PME 0.08, PLE 0.08, AMEAME 0.06, AME-ALE 0.06, PME-PME 0.16, PME-PLE 0.06, MOQ length 0.32, MOQ width 0.35; leg formula: $1-2-4-3$ : leg I - femur 1.61/ patella $0.88 /$ tibiae 1.24 / metatarsus $0.91 /$ tarsus $0.56 /$ total 5.20 ; II $1.38 / 0.72 / 0.88 / 0.75 / 0.51 / 4.24$; III $-0.88 / 0.48 / 0.72 / 0.48 / 0.35 / 2.91 ;$ IV $-1.12 / 0.48 / 0.72 / 0.56 / 0.40 /$ 3.28. Total body length 5.17; carapace 2.43 length, 2.33 width; opisthosoma length 2.74 ; clypeus 0.29 height; sternum 0.87 length, 0.80 width; endites 0.04 length, 0.24 width; labium 0.25 length, 0.32 width.

\section{Distribution}

Brazil (Amazonas, Espiríto Santo, São Paulo, Paraná, Santa Catarina and Rio Grande do Sul) (Fig. 18).

Kryptochroma quadrata Machado \& Viecelli gen. et sp. nov. urn:1sid:zoobank.org:act:6C2037C9-66B3-4B07-9102-E2BF009B8AC1

Figs $12-13,18,19 \mathrm{~B}, 22 \mathrm{~B}$

\section{Diagnosis}

The male of $K$. quadrata sp. nov. resembles that of $K$. pentacantha by the shape and disposition of embolus (Figs 13C, E, 19B), however, it can be distinguished from that and other species of the genus by the rounded tegulum, which also presents a retrolateral reentrance, and by the squared-tipped RTA with remarkable grooved surface (Figs 13D, F, 19B). The females have an anterior longitudinal ruckle connected to a shallow and convex fold on the epigynal plate, with no median outgrowth, gutters or septum separating the copulatory openings (Figs 12C, E, 22B).

\section{Etymology}

The epithet - a Latin feminine adjective that means squared - is a direct reference to the shape of the RTA tip, which ends abruptly.

\section{Material examined}

\section{Holotype}

BRAZIL • đ'; Amazonas, Coari, Porto Urucu, Base de Operações Geólogo Pedro de Moura; 4 53'42.5" S, 65¹1'18.2" W; 28 Sep. 2006; C.A.C. Santos Jr. leg.; UFMG 22673.

\section{Paratypes}

BRAZIL • 1 q; same collection data as for holotype; MPEG 22669 19 ; same locality as for preceding; $4^{\circ} 50^{\prime} 01^{\prime \prime} \mathrm{S}, 65^{\circ} 03^{\prime} 53^{\prime \prime} \mathrm{W}$; 28 Sep. 2006; D. Guimarães leg.; UFMG 22673 • 1 q, same collection data as for preceding; Sep. 2006; D. Candiani leg.; MPEG 13297. 


\section{Additional material}

None.

\section{Description}

Female (paratype, MPEG 13297)

Prosoma. Anterior eye row strongly recurved and posterior procurve, ocular mounds discrete, not as elevated as in other species, carapace predominantly yellow, black on the sides, MS well developed (Fig. 12A-B). Chelicerae yellow with two pairs of dark stains, sternum slightly longer than wide, brown with a large yellow stain, endites and labium truncated and totally brown.

LEGS. Leg coloration pattern as in K. gigas gen. et sp. nov.

Opisthosoma. Concave anterior border, predominantly yellow, dark-brown on sides and on the back of the abdominal projections (Fig. 12A). Spermathecae rounded and smooth surfaced (Fig. 12D, F).

MEAsurements. Eye diameters and eye interdistances: AME 0.06, ALE 0.11, PME 0.08, PLE 0.08, AME-AME 0.12, AME-ALE 0.16, PME-PME 0.27, PME-PLE 0.09, MOQ length 0.48, MOQ width 0.51 ; leg formula: $1-2-4-3$ : leg I - femur 5.18/ patella 2.59 / tibiae $3.47 /$ metatarsus $2.07 /$ tarsus 1.39/ total 14.70; II - 4.14/ 2.33/ 2.84/ 1.81/ 1.29/ 9.57; III - 2.33/ 1.29/2.07/ 1.03/ 0.88/ 7.60; IV $2.90 / 1.29 / 1.91 / 1.24 / 0.93 / 8.27$. Total body length 7.25 ; carapace 3.50 length, 3.37 wide; opisthosoma

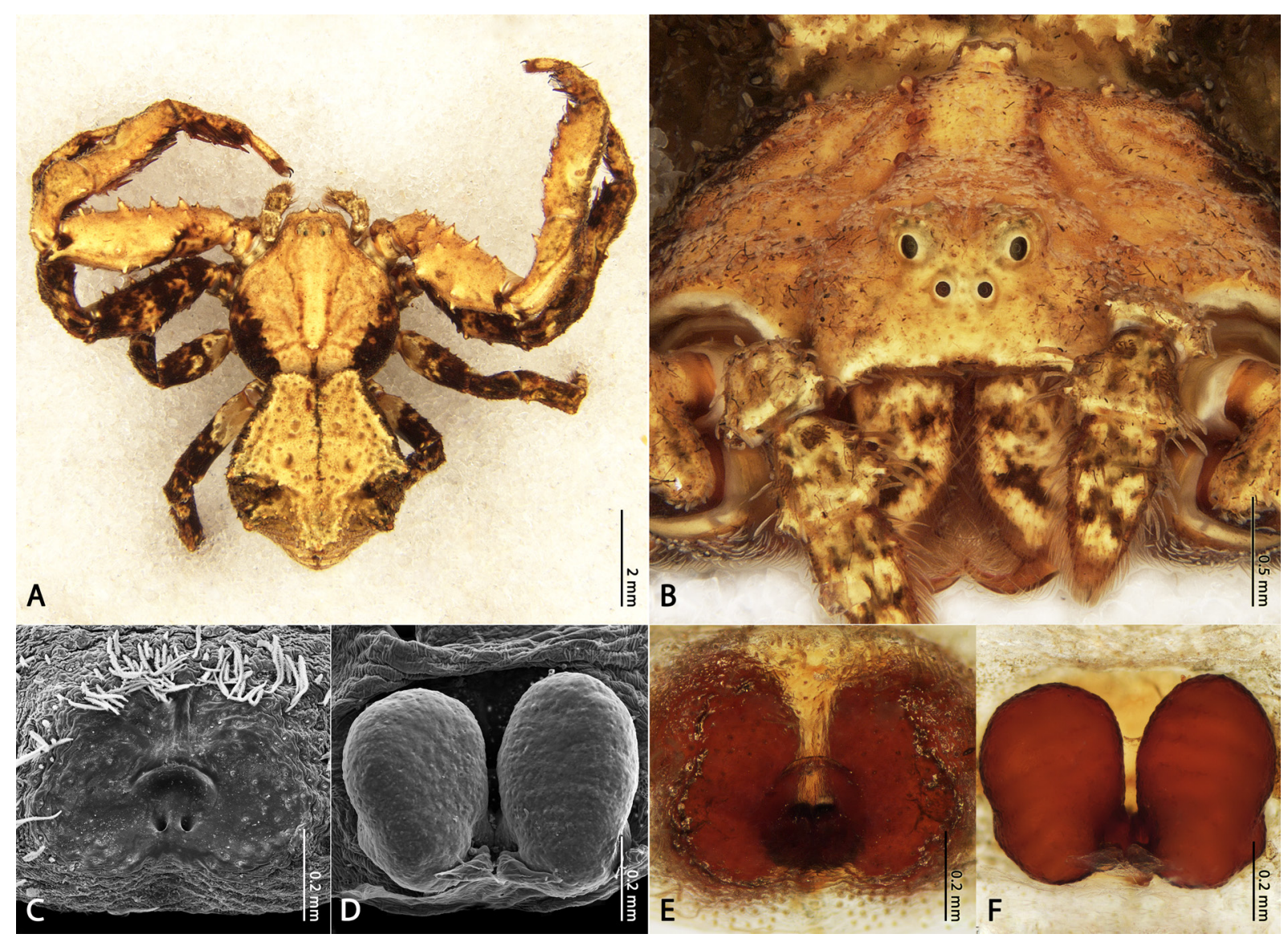

Fig. 12. Female of Kryptochroma quadrata Machado \& Viecelli gen. et sp. nov., paratype (MPEG 13297). A. Habitus, dorsal view. B. Front. C, E. Epigynal plate, ventral view. D, F. Spermathecae, dorsal view. 
length 3.75; clypeus 0.32 height; sternum 1.53 length, 1.29 width; endites 0.80 length, 0.40 width; labium 0.56 length, 0.58 width.

Male (holotype, MPEG 22673)

Prosoma. Anterior eye row very recurved, posterior procurved, ALE with almost two times the size of AME; carapace knobby, predominantly light brown with its median region, clypeus and posterior slope scars yellowish; medial spire absent (Fig. 13A-B). Sternum scutiform, light brown with a median white stain.

LEGS. Anterior legs light brown with some darker taints while legs III and IV are lighter, with extensive whitish areas; anterior femora enlarged, with dorsolateral setiferous tubercles randomly distributed and ventral region dark-brown; tibia I and II with four pairs of ventral macrosetae and anterior metatarsi with three pairs (Fig. 13A).

Opisthosoma. Predominantly pale yellow with small white punctuations and posterior black spots; straight anterior border and five short spiniform projections.

MEASUREMENTs. Eye diameters and eye interdistances: AME 0.06, ALE 0.08, PME 0.06, PLE 0.06, AMEAME 0.06, AME-ALE 0.08, PME-PME 0.16, PME-PLE 0.06, MOQ length 0.29, MOQ width 0.32; leg formula: 1-2-4-3: leg I - femur 2.23/ patella 1.24 / tibiae 1.33 / metatarsus 0.88 / tarsus 0.56 / total 6.24 ; II -
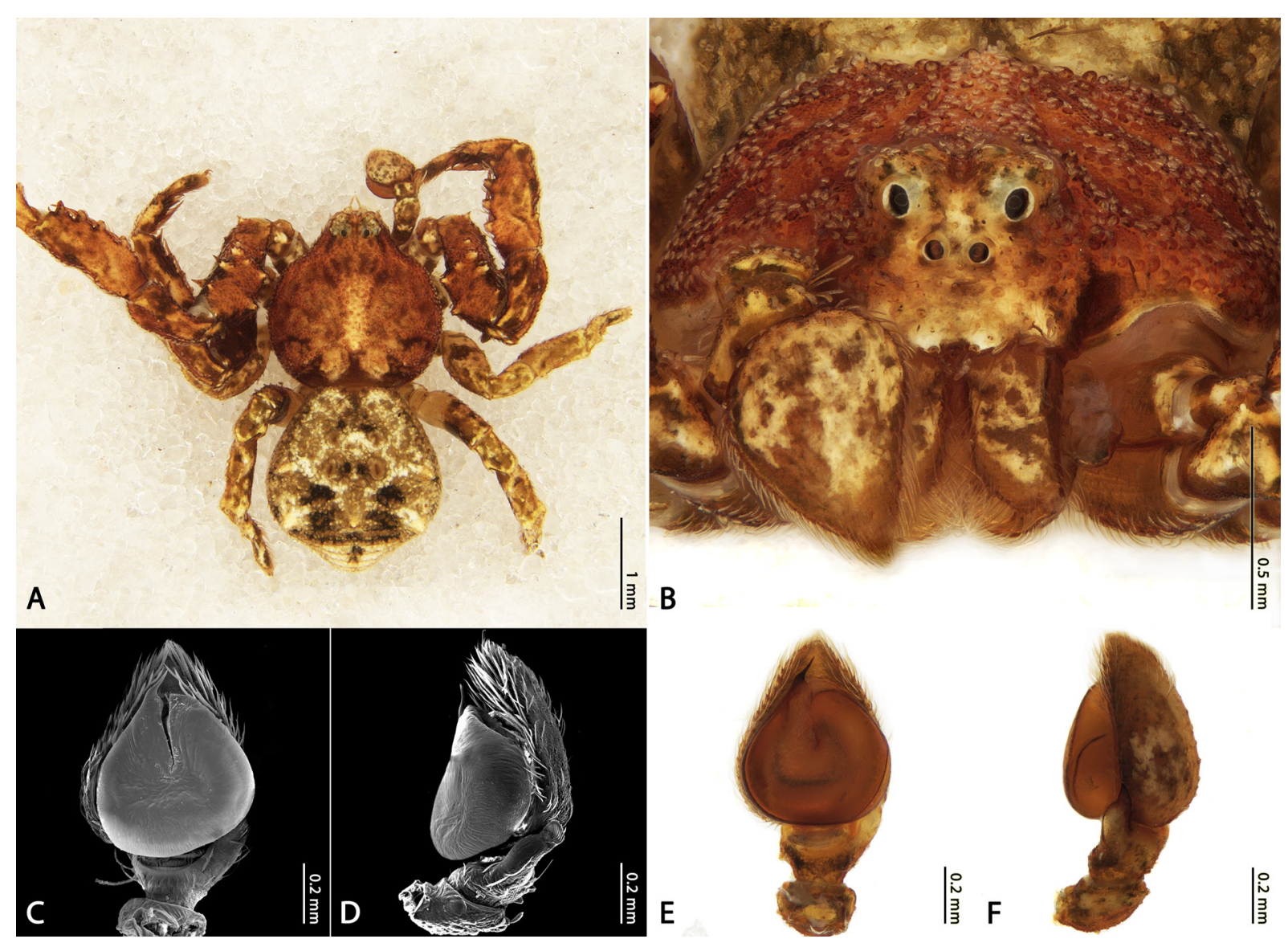

Fig. 13. Male of Kryptochroma quadrata Machado \& Viecelli gen. et sp. nov., holotype (MPEG 22673). A. Habitus, dorsal view. B. Front. C-F. Left male palp. C, E. Ventral view. D, F. Retrolateral view. 
$1.45 / 0.72 / 1.04 / 0.72 / 0.50 / 4.43 ; \mathrm{III}-0.87 / 0.48 / 0.80 / 0.45 / 0.37 / 2.97$;V $-1.12 / 0.48 / 0.80 / 0.56 / 0.40 /$ 3.36. Total body length 4.61 ; carapace 2.28 length, 2.18 width; opisthosoma length 2.33 ; clypeus 0.24 height; sternum 0.83 length, 0.72 width; endites 0.40 length, 0.24 width; labium 0.20 length, 0.32 width.

\section{Distribution}

Brazil (Amazonas) (Fig. 18).

Kryptochroma quinquetuberculata (Taczanowski, 1872) gen. et comb. nov. Figs 14, 18, 19C

Thomisus quinquetuberculatus Taczanowski, 1872: 100.

Stephanopis acanthogastra Mello-Leitão, 1929: 53, fig. 135. syn. nov.

Stephanopis quinquetuberculata - Keyserling 1880: 171, pl. 3 fig. 94. — Mello-Leitão 1929: 62. Tobias quinquetuberculatus - Simon 1895: 1053.

\section{Notes}

According to Keyserling (1880), the description of the species in his work was based on the holotype originally proposed by Taczanowski (1872), from Cayenne (French Guiana), which is presumably lost. We had access to a specimen from the Natural History Museum of London (BMNH 1890.7.1.3479) that is labeled as "type" (Fig. 14G-H); however, it is clearly not the holotype because it was collected after the original description. Since this specimen is strikingly similar to the one represented in the original illustrations (where the author highlighted the diagnostic features of the male palpus: sinuous RTA and the tip-narrowed tegulum) and was found near the original locality (New Granada, currently corresponding to the Colombian territory), we propose it as the neotype for $K$. quinquetuberculata comb. nov. (Fig. 14G-H).

\section{Diagnosis}

The male of $K$. quinquetuberculata resembles that of $K$. pentacantha by its stout opisthosomal projections and by the presence of a dorsal white macula on the opisthosoma (Fig. 14A). However, it can be recognized by the tegulum in the shape of a water drop, bearing a straight and tip-narrowed embolus instead of a curved termination observed in its closest correlated species (Figs 14C, E, 19C). Moreover, $K$. quinquetuberculata has a remarkable RTA which is large at the basis, sharpen abruptly at the tip, and sinuous, pointing towards to the dorsum of the cymbium (Figs 14D, F, 19C).

\section{Material presumably lost}

Holotype

FRENCH GUIANA • ^̊; Cayenne; MZPW.

\section{Material examined}

\section{Neotype}

COLOMBIA • ○े; BMNH 1890.7.1.3479.

Holotype of $S$. acanthogastra syn. nov. BRAZIL・ ○’; Bahia; MNHN 17126.

\section{Additional material}

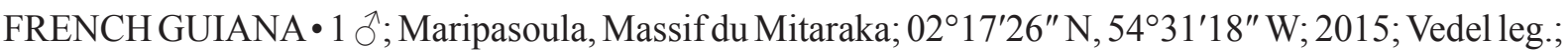

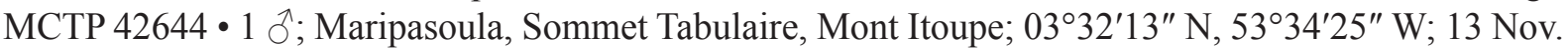
2014; Vedel and Lalague leg.; MCTP 42645. 
BRAZIL - Pernambuco State • 1 đે, 1 juv.; Recife, Horto Dois Irmãos; $8^{\circ} 00^{\prime} 35^{\prime \prime}$ S, 34 $56^{\prime} 51^{\prime \prime}$ W; 13 Sep. 1999; M. Peres leg.; IBSP 38639. - Bahia State • 1 ơ; Una, Reserva Biológica de Una; $15^{\circ} 10^{\prime} 42^{\prime \prime}$ S,

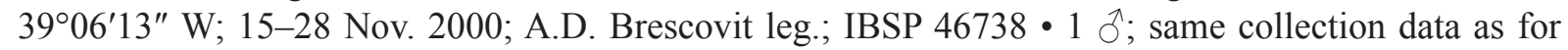
preceding; IBSP 46712 1 đ; same collection data as for preceding; IBSP $46714 \bullet 1$ đ; same collection data as for preceding; IBSP $46724 \cdot 1$ \% ; same collection data as for preceding; IBSP $46726 \bullet 1$;
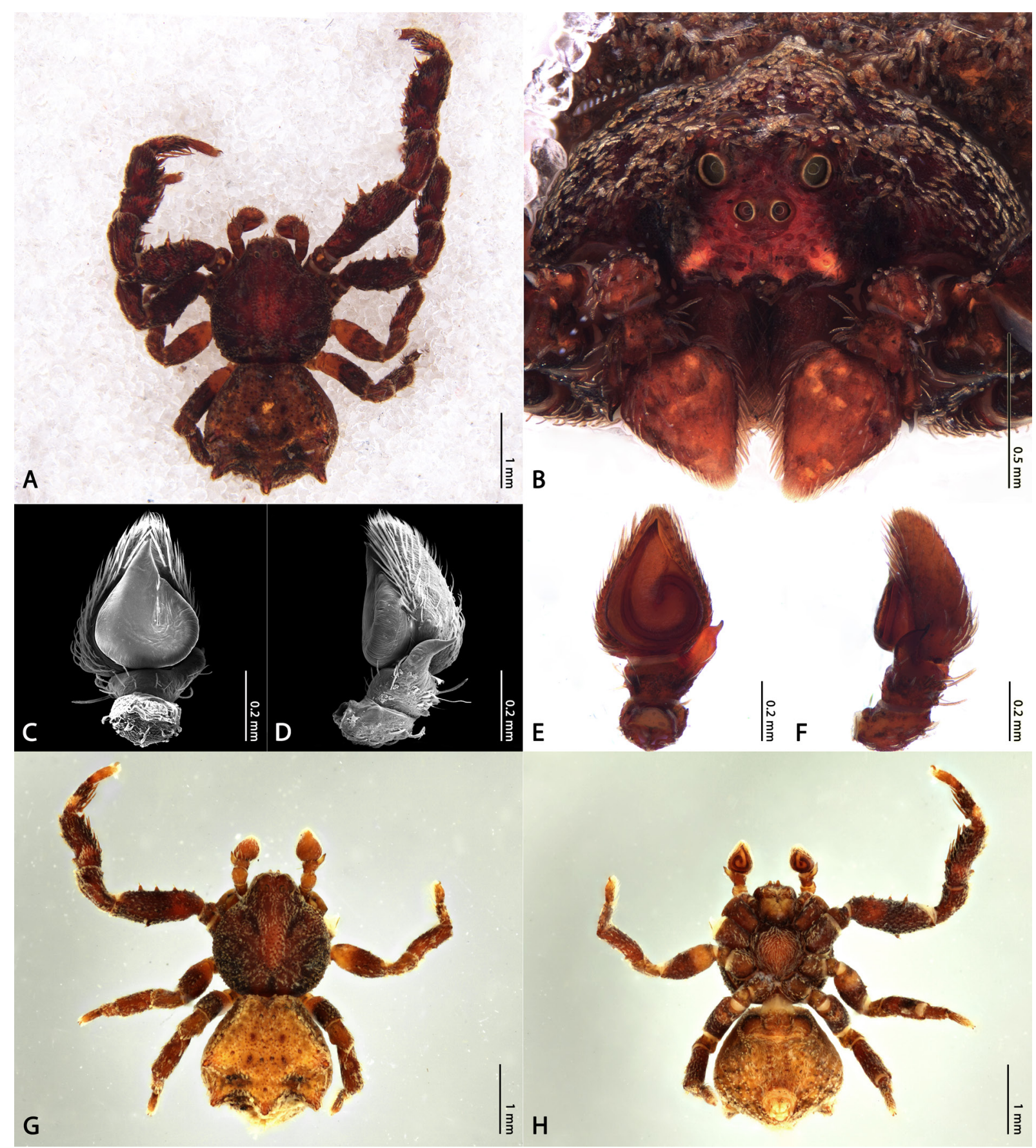

Fig. 14. Male of Kryptochroma quinquetuberculata (Taczanowski, 1872) gen. et comb. nov. A-F. Specimen IBSP 46712. A. Habitus, dorsal view. B. Front. C-F. Left male palp. C, E. Ventral view. D, F. Retrolateral view. G-H. Neotype of $K$. quinquetuberculata gen. et comb. nov. (BMNH 1890.7.1.3479). 
same collection data as for preceding; IBSP 46740 • 1 đ; Reserva Zoobotânica CEPLAC; 1446'22" S, 39¹3'13" W; 8 Dec. 2010; G.H.F. Azevedo and A.J. Santos leg.; UFMG 9484.

\section{Description}

\section{Male (IBSP 46712)}

PRosoma. Anterior eye row strongly recurved, posterior slightly procurved; carapace predominantly dark-brown with discrete MS; clypeus lighter than the rest of carapace with light spots at its lateral margins (Fig. 14B). Labium, endites and chelicerae dark-brown.

LeGs. Anterior legs (I and II) dark-brown and posterior femora (III and IV) presenting their proximal half yellow.

Opisthosoma. Predominantly brown with a dorsal white macula at its median region and a light transversal line connecting the anterolateral projections (Fig. 14A).

Measurements. Eye diameters and eye interdistances: AME 0.06, ALE 0.11, PME 0.09, PLE 0.09, AME-AME 0.06, AME-ALE 0.08, PME-PME 0.16, PME-PLE 0.04, MOQ length 0.32, MOQ width 0.35; leg formula: $1-2-4-3$ : leg I - femur 1.61 / patella 0.88 / tibiae $1.19 /$ metatarsus 0.88 / tarsus $0.56 /$ total 5.12; II - 1.45/ 0.72/ 0.96/ 0.72/ 0.48/ 4.33; III - 0.88/ 0.48/ 0.72/ 0.48/ 0.37/ 2.93; IV - 1.01/ 0.48/ $0.80 / 0.48 / 0.40 / 3.17$. Total body length 4.39 ; carapace 2.12 length, 2.07 width; opisthosoma length 2.22; clypeus 0.24 height; sternum 0.85 length, 0.72 width; endites 0.32 length, 0.25 width; labium 0.16 length, 0.29 width.

\section{Female}

Unknown.

\section{Distribution}

French Guiana (Cayenne and Maripasoula) and Brazil (Bahia and Pernambuco) (Fig. 18).

Kryptochroma renipalpis (Mello-Leitão, 1929) gen. et comb. nov.

Figs 15, 18, 19D

Stephanopis renipalpis Mello-Leitão, 1929: 60, figs 143-144.

\section{Note}

Mello-Leitão (1929) also designated a "cotype" (MNHN 17335) of S. renipalpis from Pernambuco; however, the specimen was not found.

\section{Diagnosis}

The male of $K$. renipalpis is similar to that of $K$. quinquetuberculata due to their well-developed MS, light clypeus and white macula on dorsum (Fig. 15A-B). However, it can be distinguished from this and other congeneric species by the medially positioned spermatic duct (Figs 15C, E, 19D) and extreme curvature of the RTA, which is thinner than in the other species and smooth at the tip (Figs 15D, F, 19D).

\section{Material examined}

Holotype

BRAZIL• \} \text { ; Rio de Janeiro; MNHN } 6 9 4 5 .

\section{Additional material}

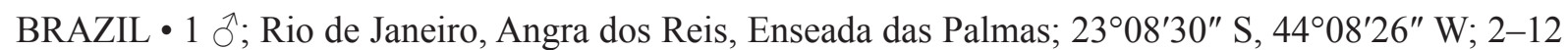
Feb. 1997; M. Ramírez leg.; MCTP 42730. 


\section{Description}

Male (MCTP 42730)

Prosoma. Anterior row eye strongly recurved and posterior slightly procurved (Fig. 15A-B); carapace predominantly reddish-brown with clypeus whitish (Fig. 15B); sternum slightly longer than wide, entirely reddish-brown; labium truncated and totally dark-brown; chelicerae reddish-brown with distal lighter spots.

LEGs. Anterior legs (I and II) mostly reddish-brown, with yellowish spots on the proximal portion of metatarsi and tarsi; posterior legs (III and IV) with bicolor femora.

Opisthosoma. Rough surfaced, dark-yellow with red spiniform projections (Fig. 15A).

Measurements. Eye diameters and eye interdistances: AME 0.06, ALE 0.09, PME 0.08, PLE 0.08, AME-AME 0.08, AME-ALE 0.06, PME-PME 0.12, PME-PLE 0.04,MOQ length 0.27, MOQ width 0.37; leg formula: $1-2-4-3$ :leg I - femur 1.91 / patella 21.03 / tibiae $1.29 /$ metatarsus $1.03 /$ tarsus $0.67 /$ total 5.93; II - 1.29/ 0.67/ 0.88/ 0.70/ 0.48/ 4.02; III - 0.85/ 0.45/ 0.64/ 0.41/ 0.32/ 2.67; IV - 0.91/ 0.40/ $0.72 / 0.51 / 0.37 / 2.91$. Total body length 4.24; carapace 2.07 length, 1.96 width; opisthosoma length 2.17; clypeus 0.19 height; sternum 0.74 length, 0.67 width; endites 0.38 length, 0.29 width; labium 0.22 length, 0.29 width.
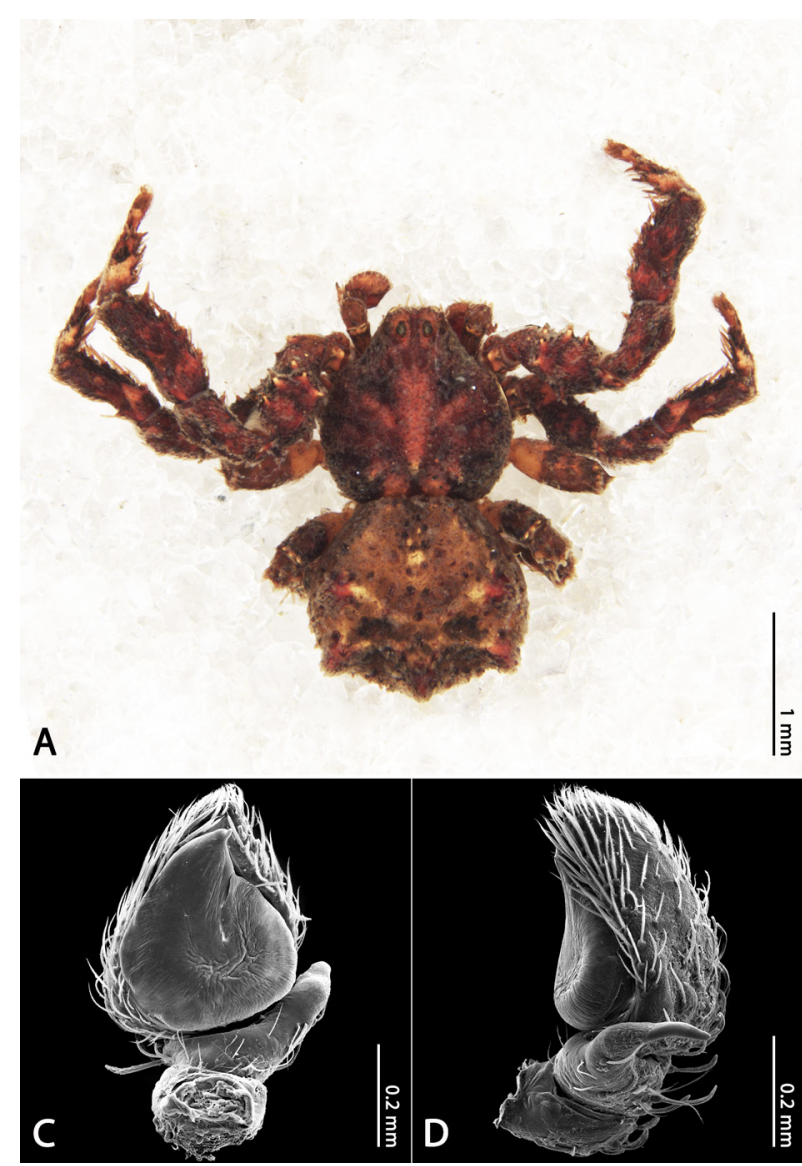
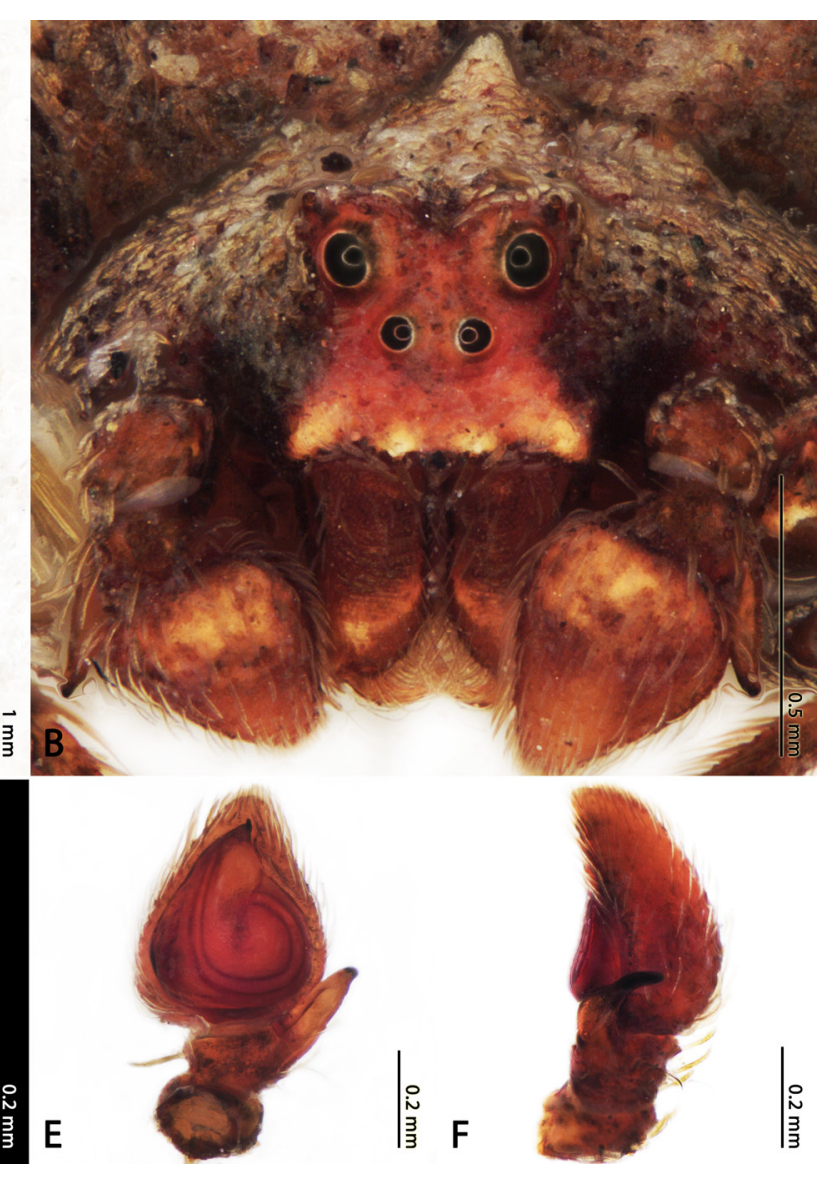

Fig. 15. Male of Kryptochroma renipalpis (Mello-Leitão, 1929) gen. et comb. nov. (MCTP 42730). A. Habitus, dorsal view. B. Front. C-F. Left male palp. C, E. Ventral view. D, F. Retrolateral view. 


\section{Female \\ Unknown. \\ Distribution \\ Brazil (Rio de Janeiro) (Fig. 18).}

Kryptochroma septata Machado \& Teixeira gen. et sp. nov. urn:lsid:zoobank.org:act:96E641ED-BE48-4D11-A39B-DED89D51FE29

Figs 16-18, 20A, 22C

\section{Diagnosis}

The female of $K$. septata sp. nov. is similar to that of $K$. pentacantha by its general body shape, prominent MS and well-developed median opisthosomal projection. However, females of K. septata sp. nov. can be recognized by the large median septum on the epigynal plate and by the deep excavations where the elliptical copulatory openings are located (Figs 16C, E, 22C). The male is similar to that of S. quadrata sp. nov. by the rounded tegulum and to $K$. pentacantha by the stout and conical RTA, however, can be distinguished from these two species by its shorter, thicker and more curved embolus (Figs 17C, E, 20A). Differently from $K$. pentacantha, in males of $K$. septata sp. nov. the RTA presents a terminal curvature that makes this apophysis points vertically rather to the backwards of the cymbium (Figs 17D, F, 20A).

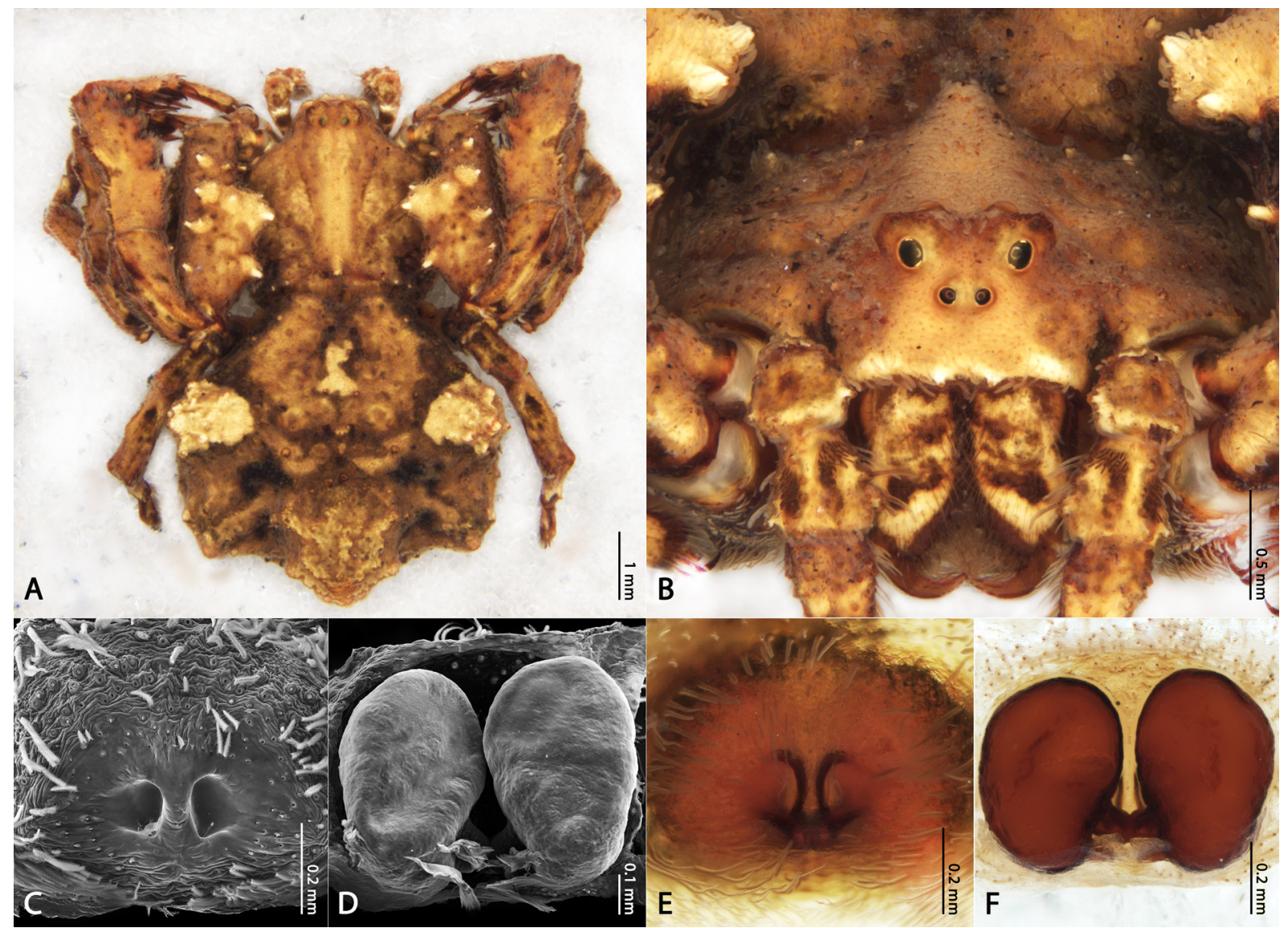

Fig. 16. Kryptochroma septata Machado \& Teixeira gen. et sp. nov., holotype, $q$ (UFMG 18269). A. Habitus, dorsal view. B. Front. C, E. Epigynal plate, ventral view. D, F. Spermathecae, dorsal view. 


\section{Etymology}

The name is a reference to the remarkable median septum on the epigynal plate of females.

\section{Material examined}

\section{Holotype}

BRAZIL • $O$; Minas Gerais, Vale Verde, Parque Nacional do Caparaó; 20²5'5.9" S, 41 $50^{\circ}$ '48.7" W; 24-30 Nov. 2014; B.T. Faleiro leg.; UFMG 18269.

\section{Paratype}

BRAZIL・1 §; same collection data as for holotype; UFMG 22431.

\section{Additional material}

None.

\section{Description}

Female (holotype, UFMG 18296)

Prosoma. Anterior row eye strongly recurved and posterior slightly procurved (Fig. 16B); ALE slightly larger than AME; carapace predominantly dark-yellow with cephalic region dark-brown; clypeus yellow; sternum slightly longer than wide, dark-brown with a central yellow stain; labium truncated and totally dark-brown; chelicerae dark-yellow
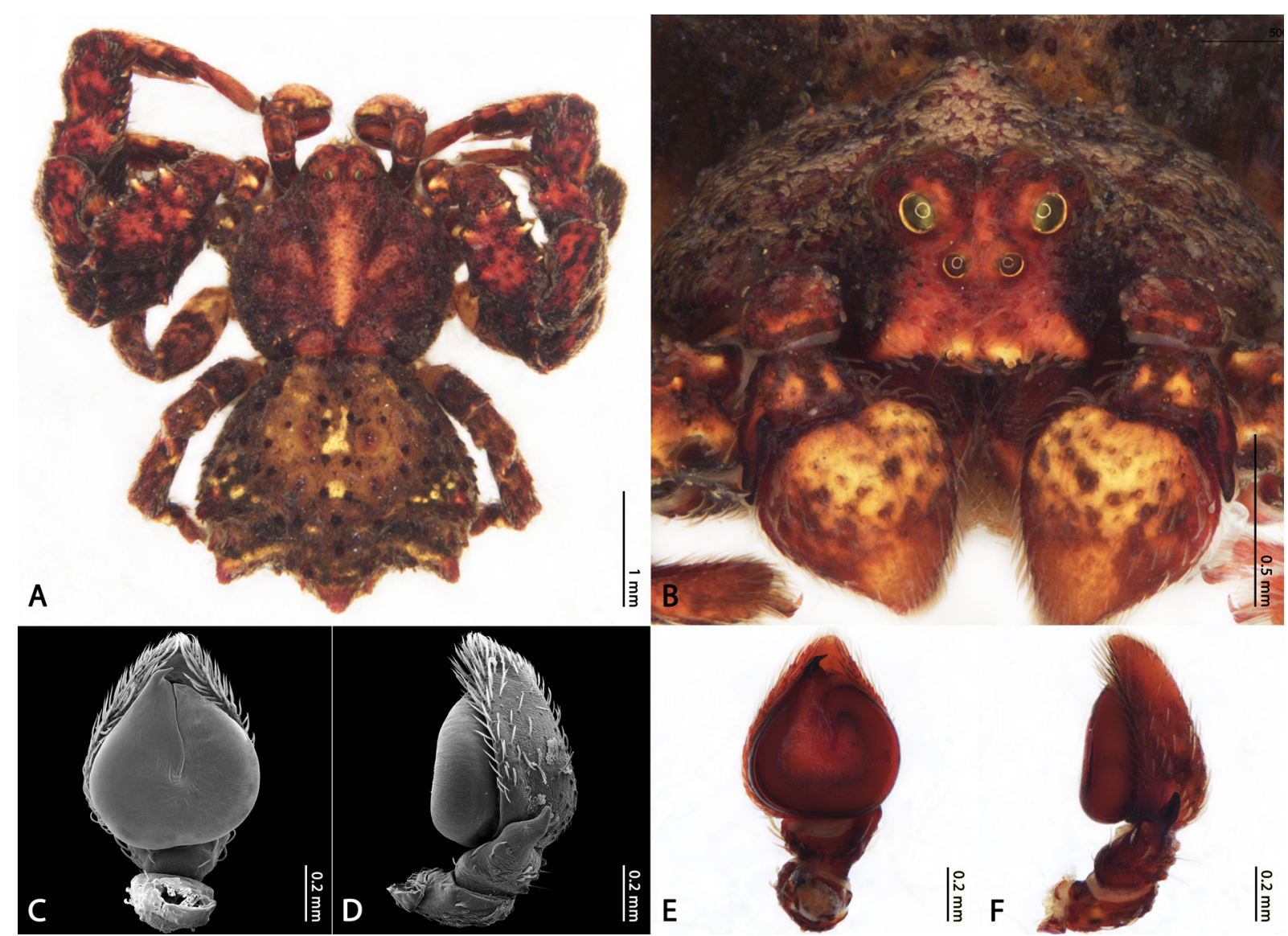

Fig. 17. Male of Kryptochroma septata Machado \& Teixeira gen. et sp. nov., paratype (22431). A. Habitus, dorsal view. B. Front. C-F. Left male palp. C, E. Ventral view. D, F. Retrolateral view. 
LeGs. Anterior legs (I and II) dark-yellow with dark taints randomly distributed; posterior legs (III and IV) predominantly yellow.

Opisthosoma. Rough surfaced, predominantly dark-yellow, with dark-brown stains bypassing the upperlateral opisthosomal projections and the median posterior one; a central white macula can be observed on the dorsum (Fig. 16A); spermathecae smooth surfaced and kidney-shaped (Fig. 16D, F).

MEAsurements. Eye diameters and eye interdistances: AME 0.10, ALE 0.16, PME 0.14, PLE 0.14,AMEAME 0.12, AME-ALE 0.14, PME-PME 0.22, PME-PLE 0.07, MOQ length 0.12, MOQ width 0.20; leg formula: 1-2-4-3: leg I - femur 4/ patella 2.15/ tibiae 2.75/ metatarsus 1.75/ tarsus 1.15/ total 11.80; II - 3.25/ 1.80/ 2.25/ 1.50/ 1.15/ 9.95; III - 1.75/ 1/ 1.50/ 0.90/ 0.90/ 6.05; IV - 2.25/ 0.75/ 1.75/ 1/ 0.80/

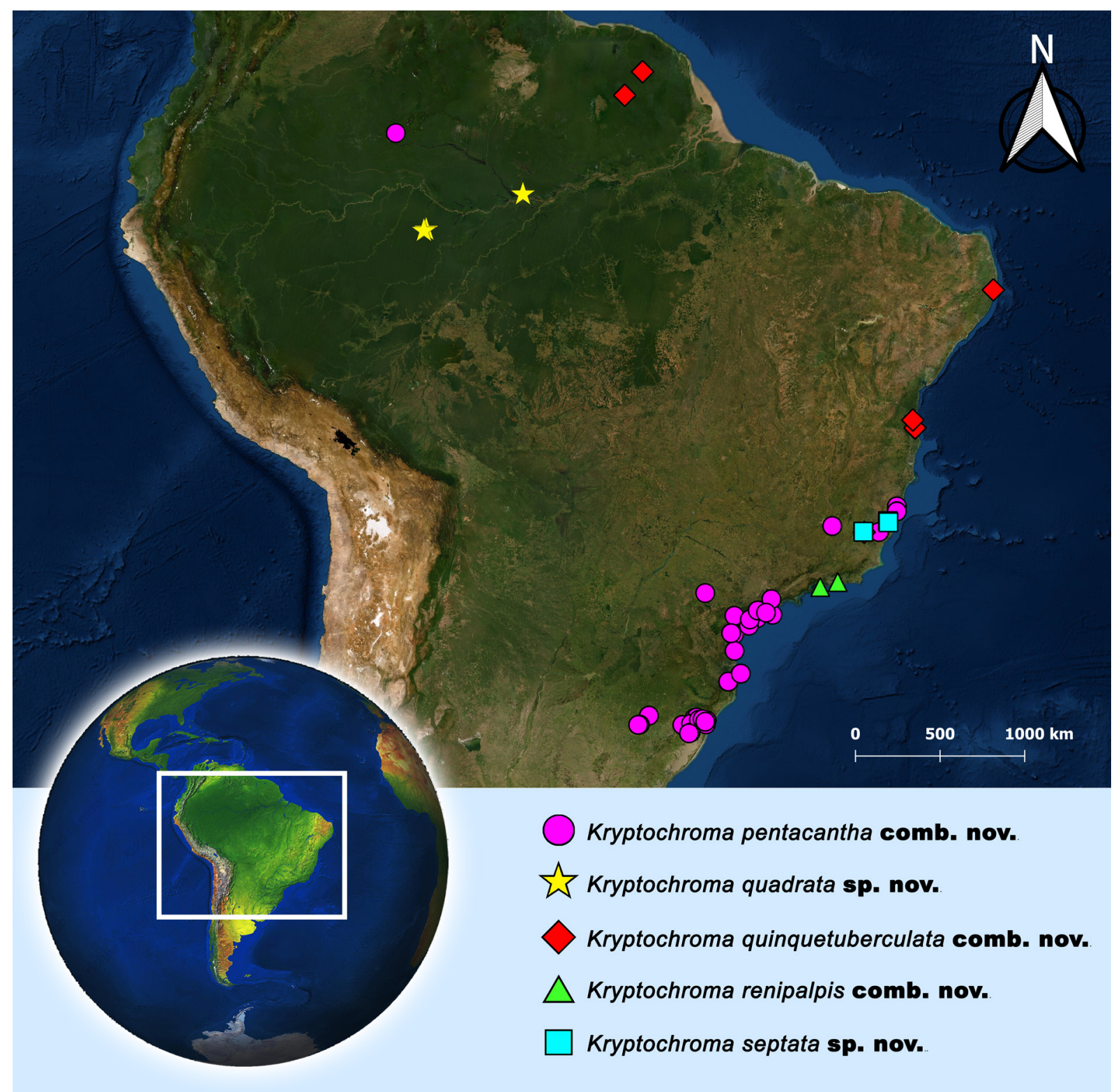

Fig. 18. Distribution records of species of Kryptochroma Machado gen. nov. 
6.55. Total body length 7.80 ; carapace 3.65 length, 3.75 width; opisthosoma length 4.15 ; clypeus 0.40 height; sternum 1.70 length, 1.50 width; endites 0.90 length, 0.46 width; labium 0.48 length, 0.74 width.

Male (paratype, UFMG 22431)

Prosoma. Reddish-brown with lighter median area and clypeus (Fig. 17A-B).

LEGS. Anterior legs (I and II) reddish-brown with macrosetae sockets yellowish-white; posterior femora (III and IV) bicolor, yellow on their proximal half and reddish-brown on its distal part.

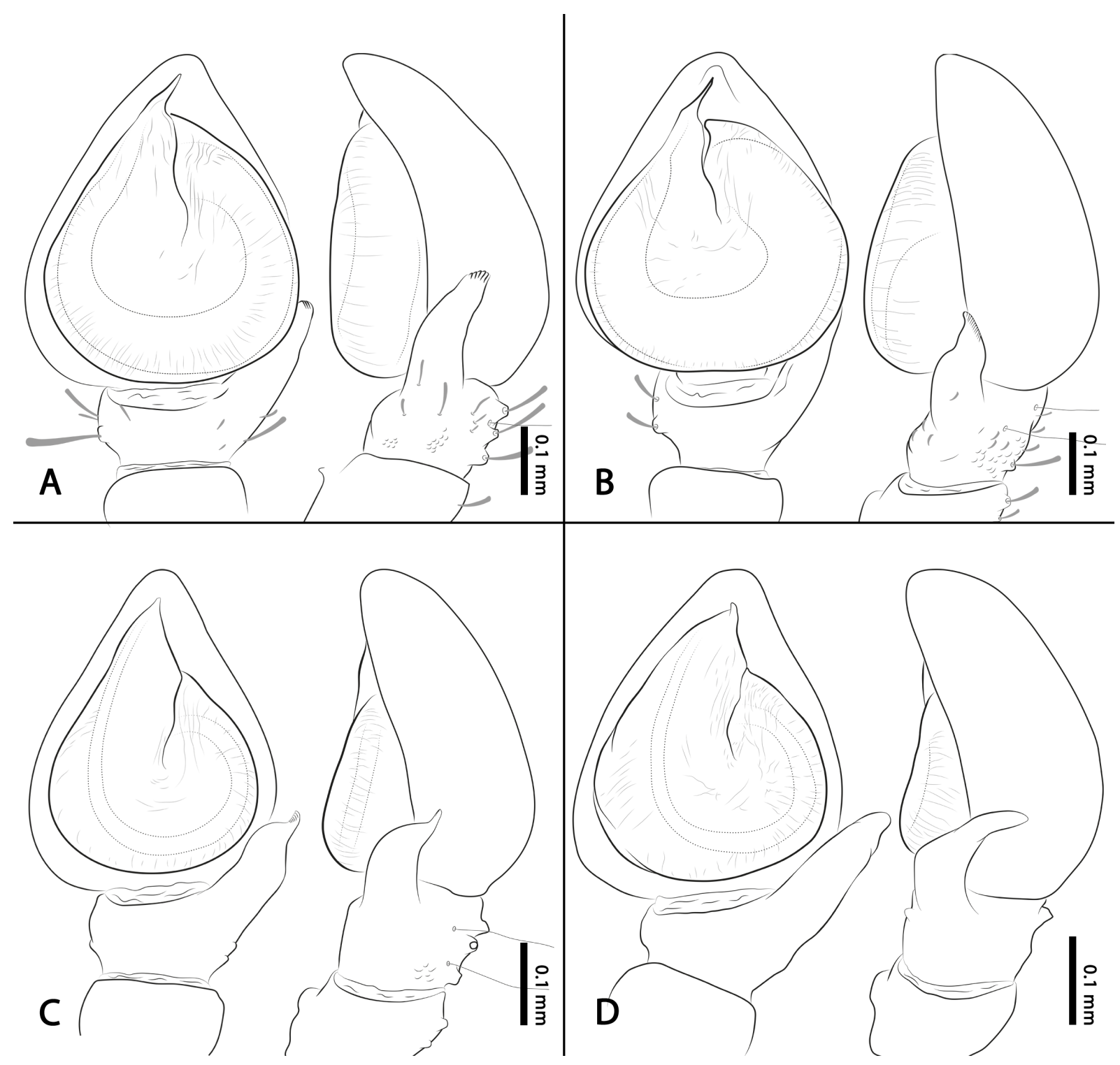

Fig. 19. Illustrations of the left male palp in ventral and retrolateral view. A. Kryptochroma pentacantha (Mello-Leitão, 1929) gen. et comb. nov. ((MCTP 25762). B. K. quadrata Machado \& Viecelli gen. et sp. nov., holotype (MPEG 22673). C. K. quinquetuberculata (Taczanowski, 1872) gen. et comb. nov. (IBSP 46712). D. Kryptochroma renipalpis (Mello-Leitão, 1929) gen. et comb. nov. (MCTP 42730). 
Opisthosoma. Predominantly brown with black punctuations; opisthosomal projections are reddish and bear white maculae on its basis; a dorsal white guanine spot can be also observed on the median region of the dorsum (Fig. 17A).

Measurements. Eye diameters and eye interdistances: AME 0.07, ALE 0.09, PME 0.07, PLE 0.07, AME-AME 0.09, AME-ALE 0.09, PME-PME 0.16, PME-PLE 0.07, MOQ length 0.28, MOQ width 0.23 ; leg formula: $1-2-4-3$ : leg I - femur 1.71 / patella 0.92 / tibiae $1.35 /$ metatarsus $1.02 /$ tarsus $0.57 /$ total 5.57; II - 1.33/ 0.83/ 1.04/ 0.80/ 0.59/4.59; III - 0.88/ 0.57/ 0.78/ 0.54/ 0.38/3.15; IV - 1.09/ 0.57/ $0.85 / 0.64 / 0.42 / 3.57$. Total body length 4.16 ; carapace 1.97 length, 1.85 width; opisthosoma length

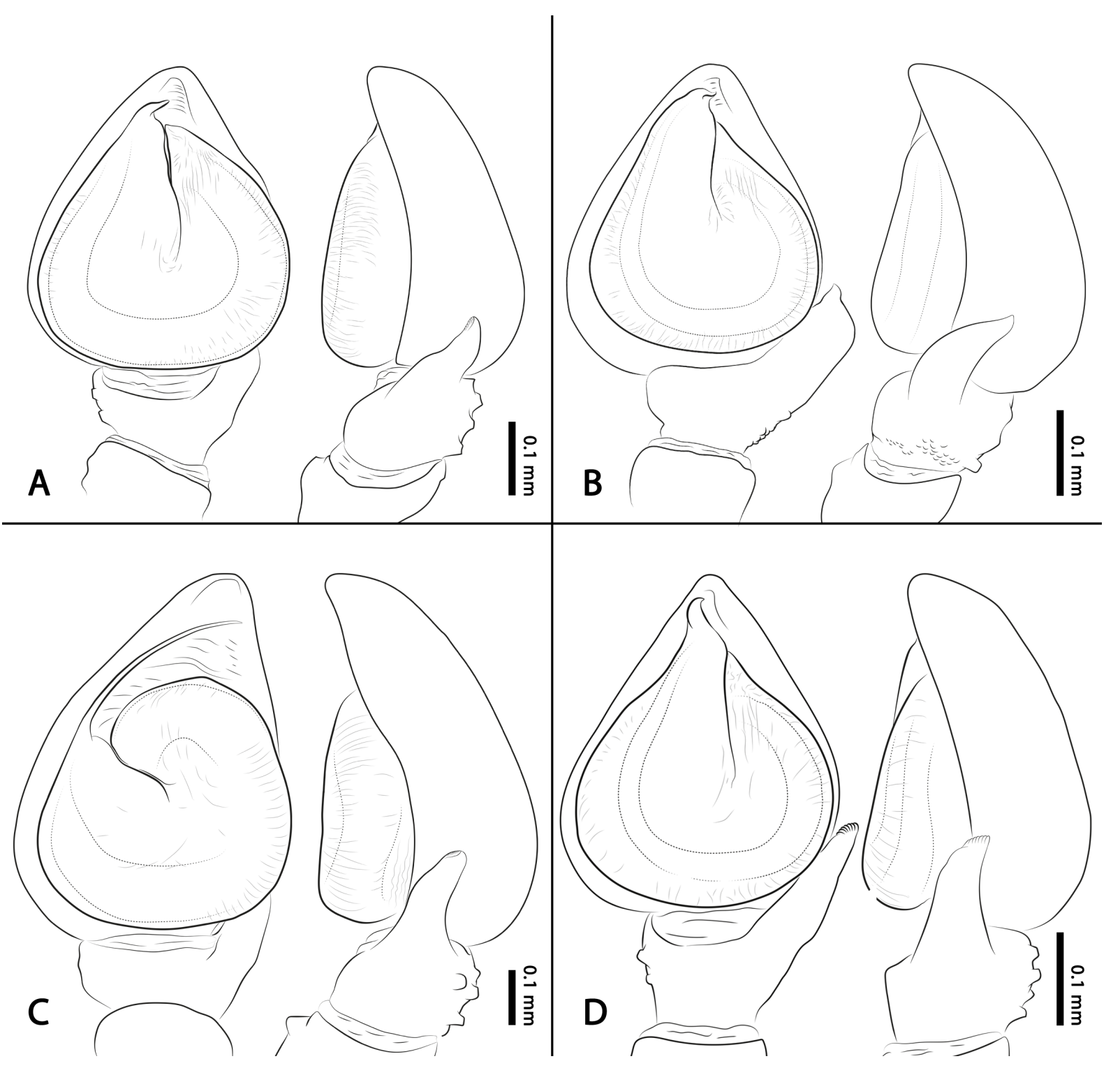

Fig. 20. Illustrations of the left male palp in ventral and retrolateral view. A. Kryptochroma septata Machado \& Teixeira gen. et sp. nov., paratype (UFMG 22431). B. K. hilaris Machado \& Teixeira gen. et sp. nov. (MPEG 13239). C. K. macrostyla (Mello-Leitão, 1929) gen. et comb. nov. (MZSP 15320). D. K. parahybana (Mello-Leitão, 1929) gen. et comb. nov. (MPEG 09069) 

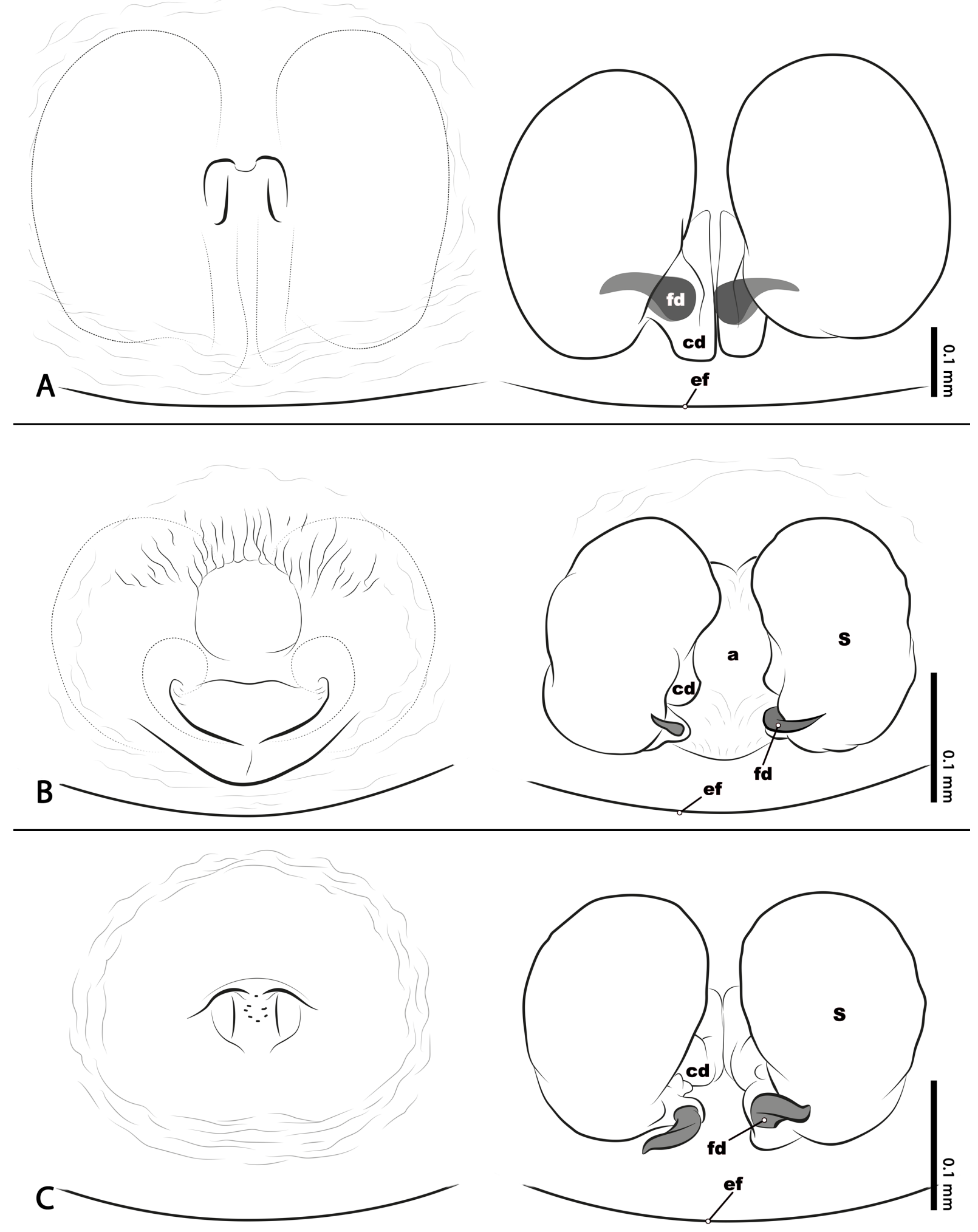

Fig. 21. Illustrations of epigynum in ventral and dorsal view. A. Kryptochroma gigas Machado \& Viecelli gen. et sp. nov., holotype (UFMG 4734). B. K. hilaris Machado \& Teixeira gen. et sp. nov., holotype (MPEG 13322). C. K. parahybana (Mello-Leitão, 1929) gen. et comb. nov. (MPEG 30339). 

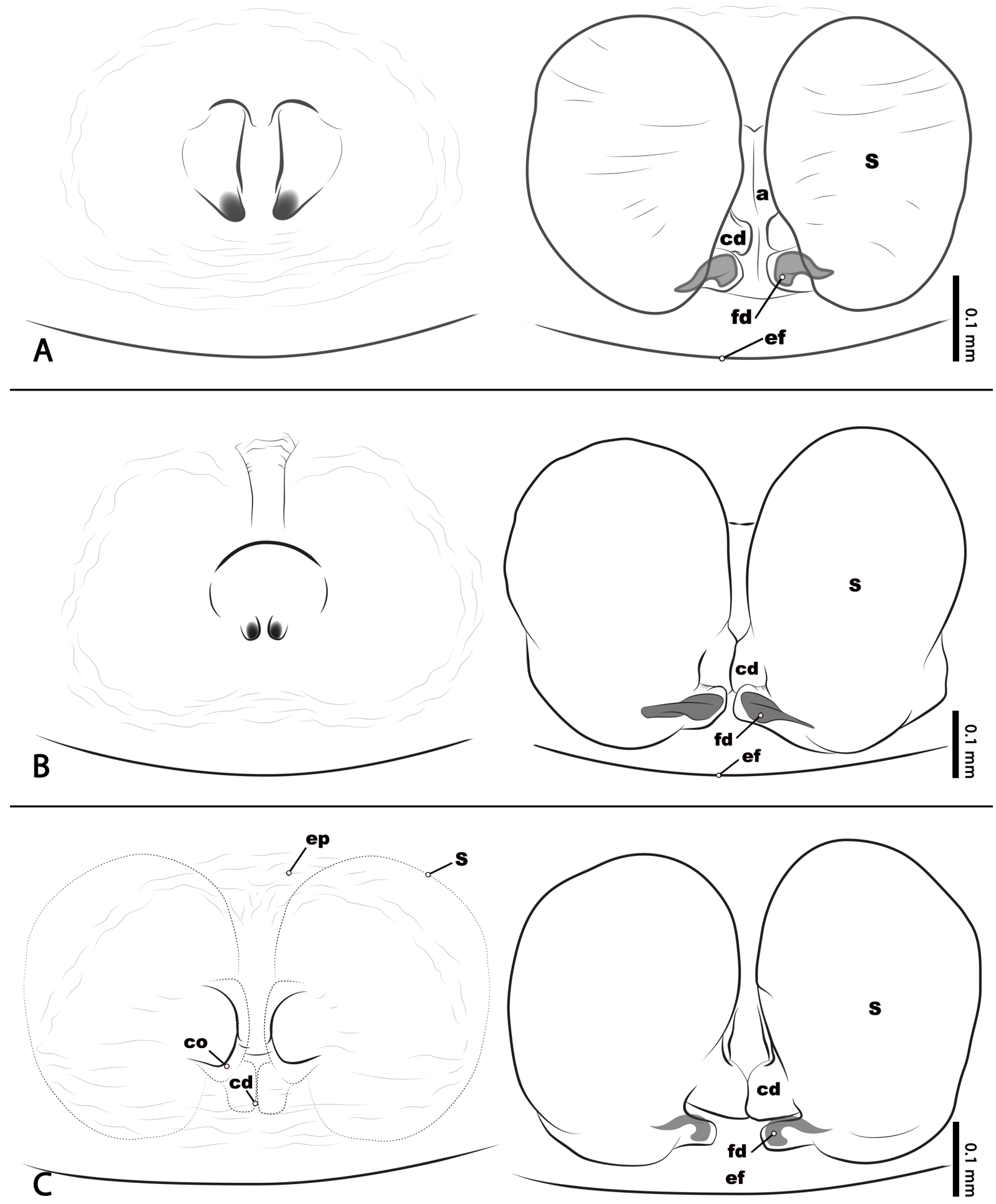

Fig. 22. Illustrations of epigynum in ventral and dorsal view. A. Kryptochroma pentacantha (MelloLeitão, 1929) gen. et comb. nov. (MCTP 38671). B. K. quadrata Machado \& Viecelli gen. et sp. nov., apratype (MPEG 13297). C. K. septata Machado \& Teixeira gen. et sp. nov., holotype (UFMG 18296). 
2.19; clypeus 0.23 height; sternum 0.88 length, 0.88 width; endites 0.40 length, 0.21 width; labium 0.21 length, 0.30 width.

\section{Distribution}

Brazil (Espírito Santo and Minas Gerais) (Fig. 18).

\section{Additional taxonomic acts}

The type material of all other Neotropical species hitherto assigned to Stephanopis was examined. The following species do not share the diagnostic characters proposed for Kryptochroma gen. nov., therefore

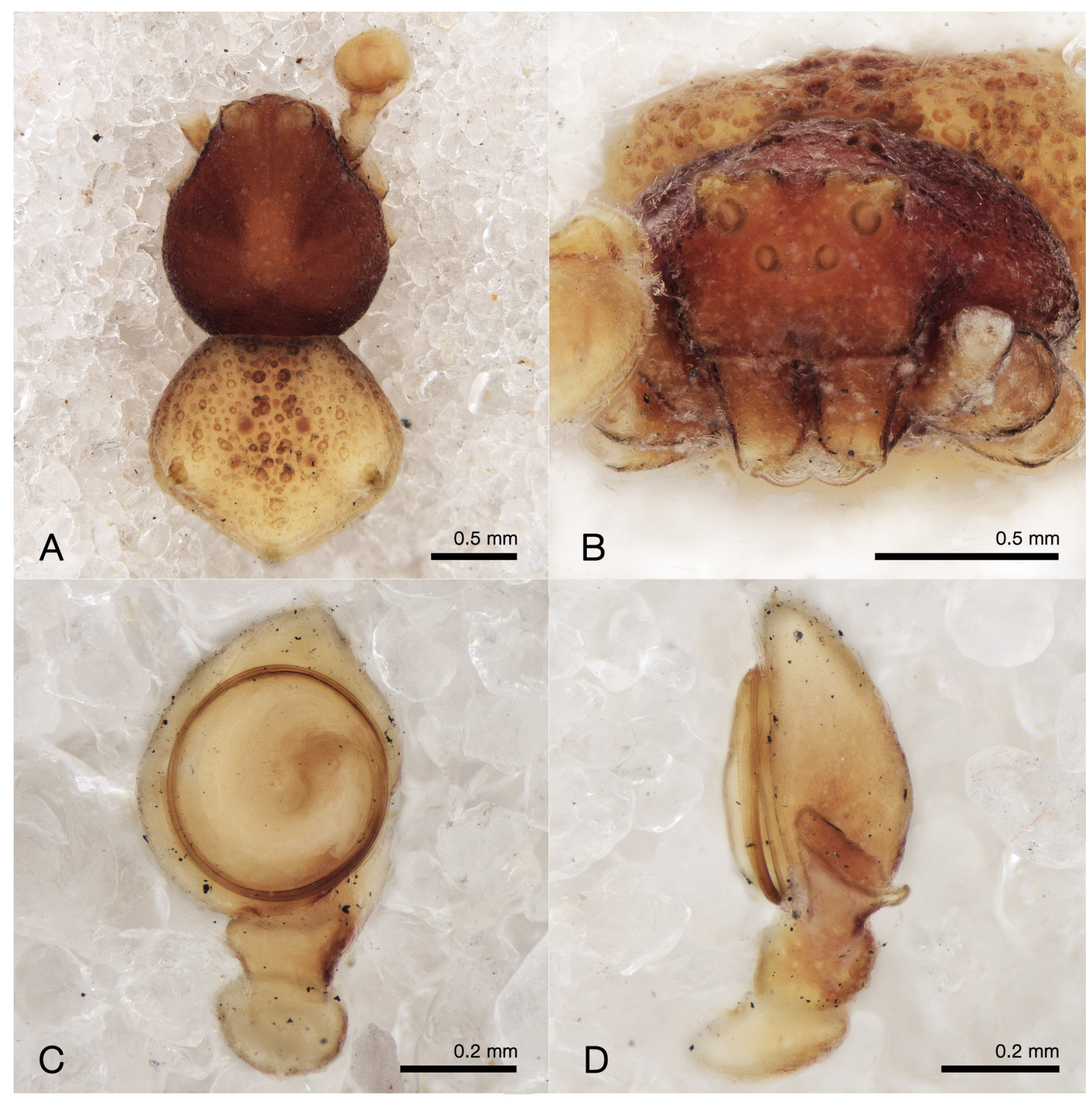

Fig. 23. Stephanopis aheneus Soares \& Soares, 1946. Holotype (MZSP 666). A. Habitus, dorsal view. B. Front. C-D. Left palp. C. Ventral view. D. Retrolateral view. 
they are being considered as nomina dubia or transferred to other genera recently revised by Teixeira et al. (2014), Silva-Moreira \& Machado (2016) and Machado et al. (2018, 2019a).

Epicadus tuberculatus (Petrunkevitch, 1910)

Stephanopis aheneus Soares \& Soares, 1946: 57, figs 5-6 (male holotype from Rio São José, Colatina, Espírito Santo, Brazil, Soares, B.A.M. leg.,15 September 1942, deposited in MZSP 666, examined). syn. nov.

\section{Note}

This synonymy is proposed based on the shape of the tegulum, RTA and the presence of tubercles on the thoracic portion of the carapace (Fig. 23) - complete synonymic list in Prado et al. (2018).

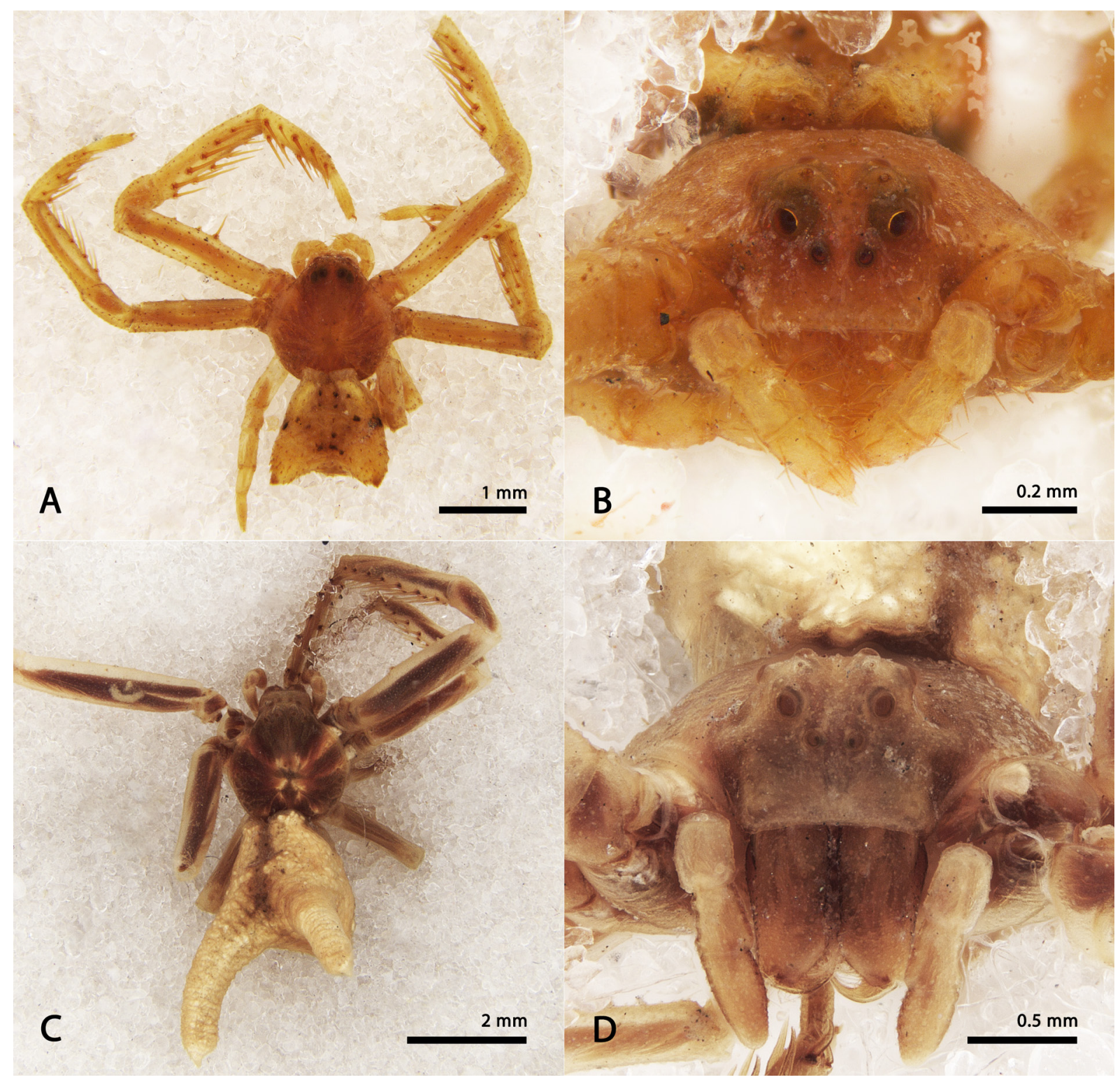

Fig. 24. A-B. Holotype of Stephanopis borgmeyeri Mello-Leitão, 1929. Holotype (MNRJ 917). A. Habitus, dorsal view. B. Front. C-D. Stephanopis furcillata Keyserling, 1880. Holotype (ZMHB 2406). C. Habitus, dorsal view. D. Front. 
Stephanopis borgmeyeri Mello-Leitão, 1929 nom. dub.

Fig. 24A-B

Stephanopis borgmeyeri Mello-Leitão, 1929: 54, fig. 40 (immature female holotype from Petrópolis, Rio de Janeiro, Brazil, deposited in MNRJ 917, examined).

\section{Note}

Proposed as nomen dubium once the immature specimen does not allow a reliable identification (Fig. 24A-B).
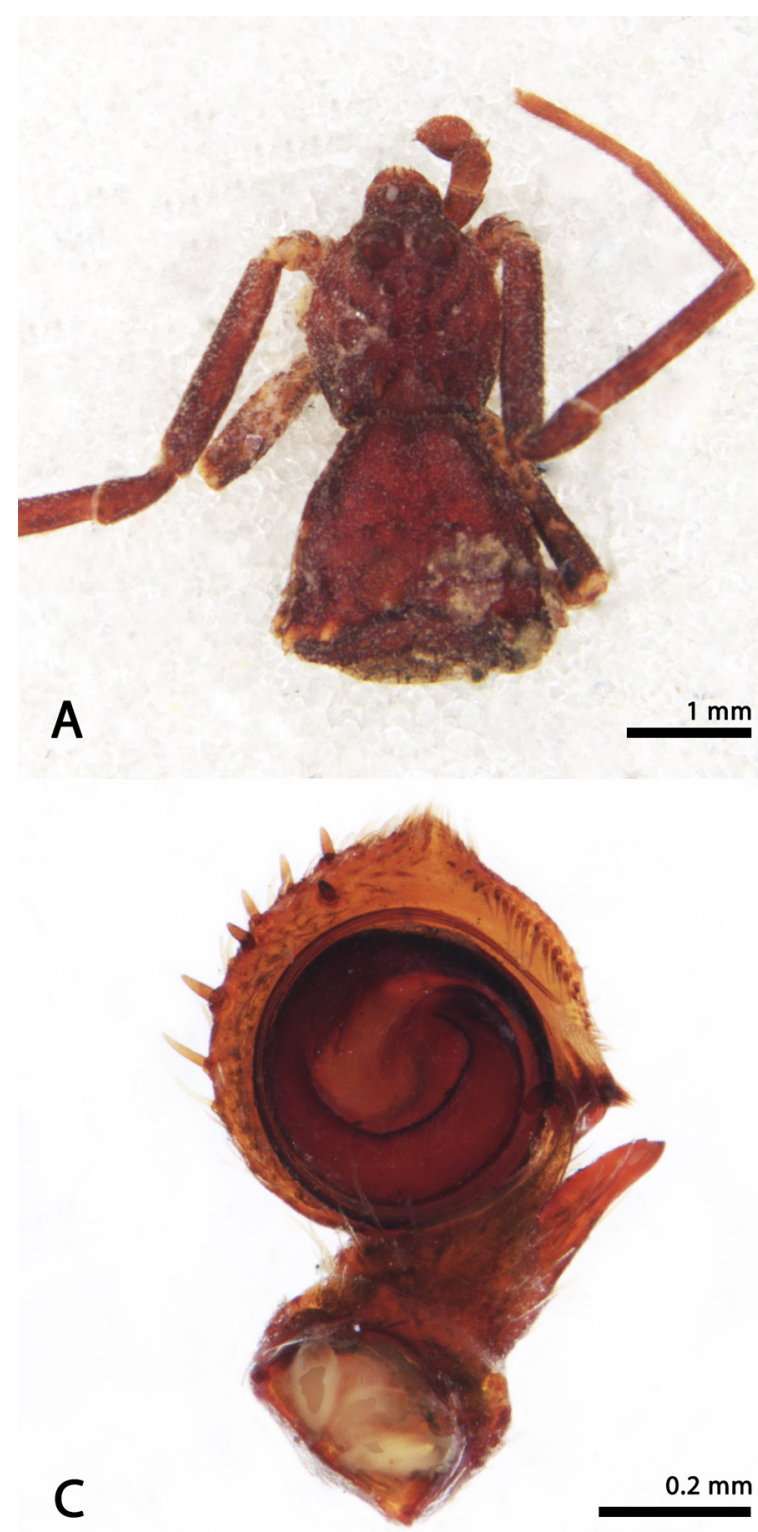
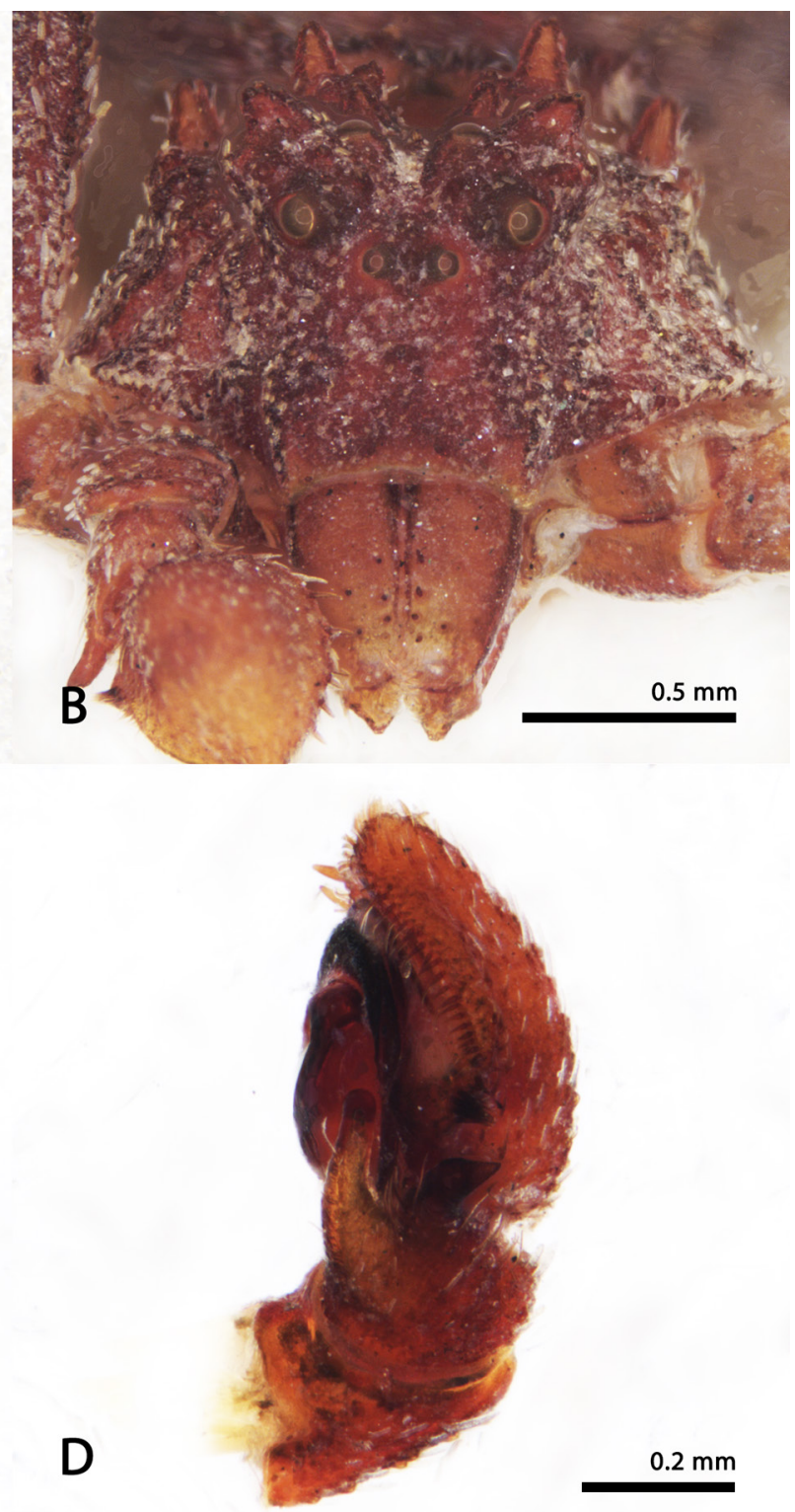

Fig. 25. Stephanopis quimiliensis Mello-Leitão, 1942. Holotype, đ̊ (MLP 15455). A. Habitus, dorsal view. B. Front. C-D. Left palp. C. Ventral view. D. Retrolateral view. 


\section{Stephanopsis furcillata Keyserling, 1880}

Stephanopis furcillata Keyserling, 1880: 179, pl. 4 fig. 98 (female holotype from Brazil, deposited in ZMHB 2406, examined).

\section{Note}

We propose Stephanopis furcillata as senior synonym of Sidymella multispinulosa (Mello-Leitão, 1944), based on the distinct opisthosoma presenting a long pair of projections with a terminal tubercle at their tips (Fig. 24C-D).

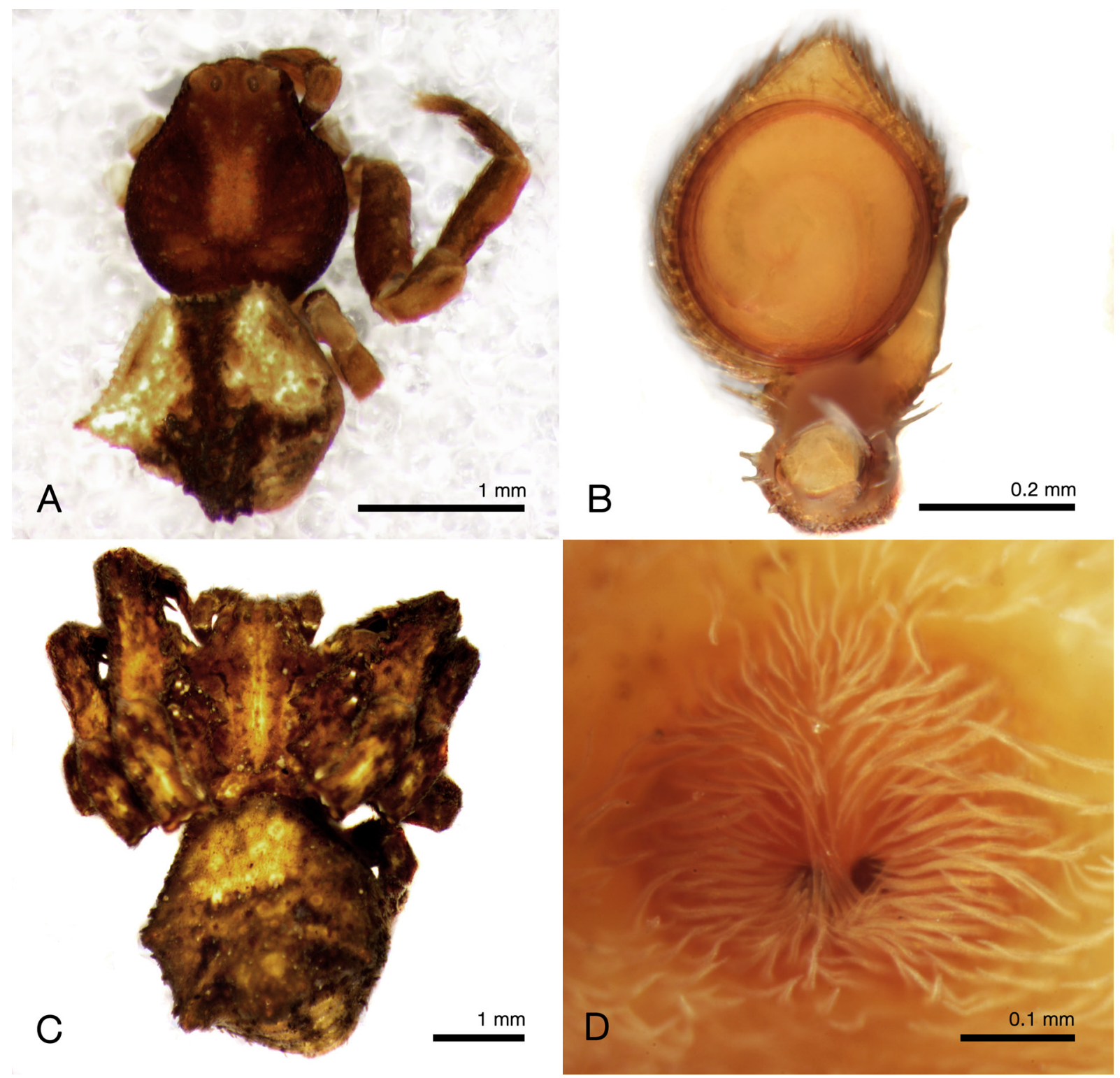

Fig. 26. Syntypes of Stephanopis salobrensis Mello-Leitão, 1929 (MNHN 3973). A. Male habitus, dorsal view. B. Left palp, ventral view. C. Female habitus, dorsal view. D. Epigynal plate, ventral view. 
Ulocymus quimiliensis (Mello-Leitão, 1942) comb. nov. Fig. 25

Stephanopis quimiliensis Mello-Leitão, 1942: 408, figs 33-34 (male holotype from Quimilí, Santiago del Estero, Argentina, Birabén, M. leg., deposited in MLP 15455, examined). syn. nov.

\section{Note}

This species lacks the typical cheliceral teeth that are still considered as a diagnostic feature for Stephanopinae. Instead, its elongated labium and peglike-setae on endites (see Teixeira et al. 2014) allow us to undoubtedly consider this spider as an Aphantochilinae. We propose the transfer of S. quimiliensis to Ulocymus Simon, 1866 based on the presence of stout and conical projections on the dorsum of the carapace (see Teixeira et al. 2014) and shape of the copulatory structures (Fig. 25).

Epicadus stelloides (Walckenaer, 1837) comb. nov.

Thomisus stelloides Walckenaer, 1837: 514 (female holotype from "Ilê Tortue", Tortuga, Haiti, deposited in MNHN, probably lost).

Stephanopis trilobata Mello-Leitão, 1929: 56, fig. 136 (female holotype collected in Goiás, Brazil, deposited in MNHN 21629, examined; female "cotype", collected in Goiás, Brazil, deposited in MNHN 21628, examined). syn. nov. (Fig. 27E-F).

Stephanopis salobrensis Mello-Leitão, 1929: 57, figs 137-139 (2 females paralectotypes, misidentified; 1 male lectotype designated here, collected from Salobro, Bahia, Brazil, deposited in MNHN 3973, examined). syn. nov. (Fig. 26).

Stephanopis stelloides - Keyserling 1880: 173 (female cotype collected from "Tortosa" by GuérinMeneville, not located). syn. nov. (Fig. 27C-D).

\section{Note}

Although the holotype described by Walckenaer (1837) is presumably lost, and the "conspecific" and sympatric specimen used by Keyserling (1880) to describe $S$. stelloides had not been found, the original descriptions and illustrations (Fig. 27C-D) allowed us to unequivocally identify this species as conspecific with Epicadus caudatus (Fig. 27A-B), a species that was recently examined and revised by Machado et al. $(2015,2017)$. Following the priority principle, we transfer Stephanopis stelloides to Epicadus and propose the name E. stelloides comb. nov. as a senior synonym over E. caudatus (Fig. 27). Thus, based on the images provided by Machado et al. (2015), we also recognize the identity of the male specimen of $S$. salobrensis as conspecific to E. stelloides comb. nov.

\section{Discussion}

The crab spiders assigned to the subfamily Stephanopinae were repetitively reported as a paraphyletic group whose recovered branches are usually unstable and weakly supported (Benjamin et al. 2008; Benjamin 2011; Ramírez 2014; Wheeler et al. 2017). This paraphyly and other insights about the taxonomic boundaries of Stephanopis were observed in the cladistic analysis provided by Machado et al. (2017). These preliminary results and seminal ideas that, at the time focused on the relationships of the genus Epicadus, led us (Machado \& Teixeira 2021) to extend our sample and investigate the relationships of Stephanopis and its presumable paraphyly. Since this genus had several species described from the Australian and Neotropical regions, and its taxonomic background included many species currently assigned to Sidymella, both genera were studied by Machado \& Teixeira (2021). The recent findings provided by these authors corroborate the hypothesis of the polyphyletism for Stephanopis. Among the distinct clades that emerged from the genus, there is a remarkable group of species with distribution restricted to the Neotropical Region, which Machado \& Teixeira (2021) named "pentacantha clade". 


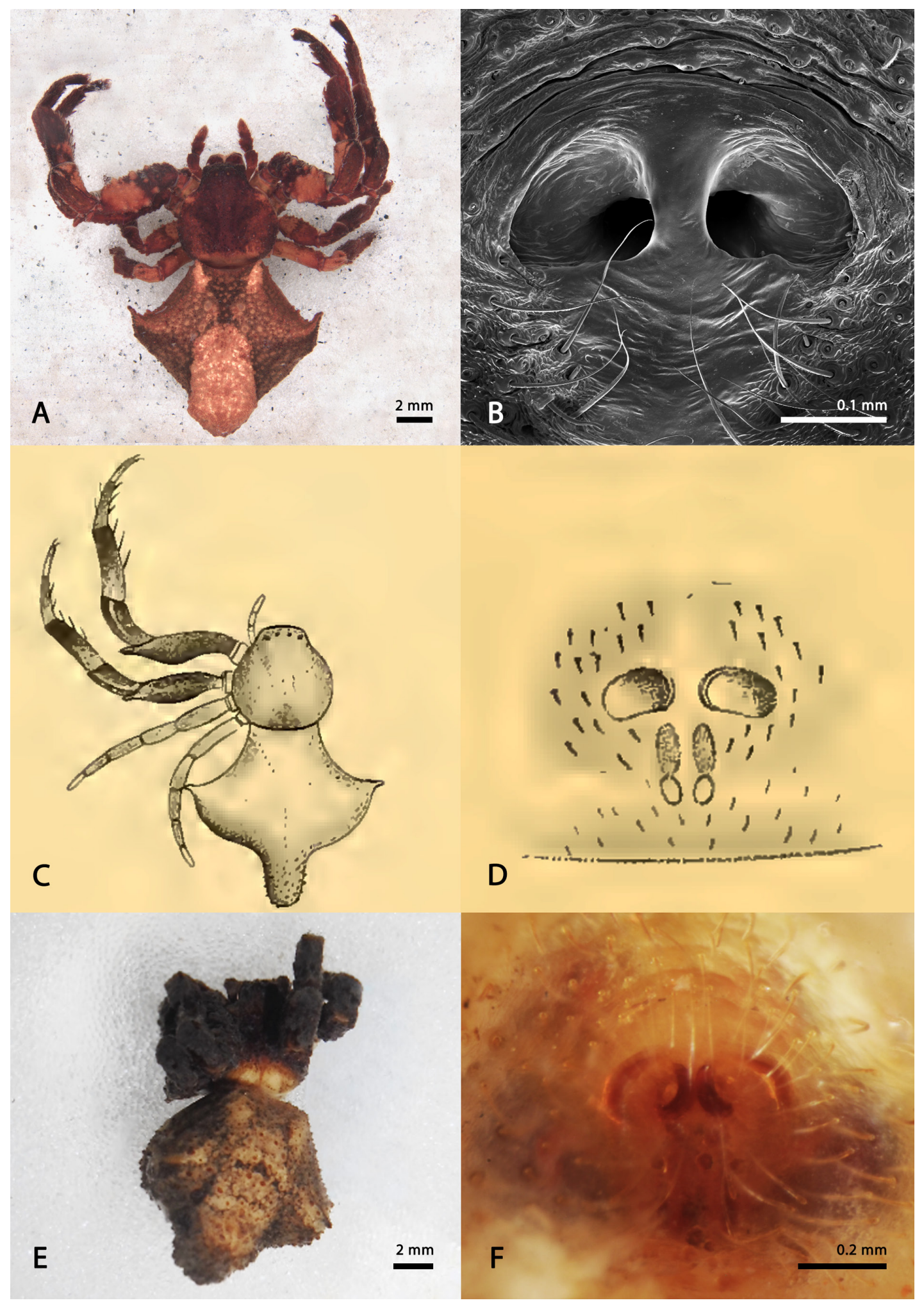

Fig. 27. A-B. Female of Epicadus caudatus (Mello-Leitão, 1929) (MCTP 7593) for comparison. A. Habitus, dorsal view. B. Epigynal plate, ventral view. - C-D. Sketches from the original description of Stephanopis stelloides (Walckenaer, 1837). C. Habitus, dorsal view. D. Epigynal plate, ventral view. - E-F. Stephanopis trilobata Mello-Leitão, 1929. Holotype, o (MNHN 21629). E. Habitus, dorsal view. F. Epigynal plate, ventral view. 
This group was recovered with significant stability and branch support, presenting two synapomorphies: (1) patellae I and II with a pair of ventral macrosetae; (2) pear-shaped tegulum. Here, we not only describe and provide detailed images of these traits but also formally propose a new generic assignment to this group of species provisionally treated as a clade that should not be recognized as part of Stephanopis (stricto sensu).

Although these studies were based solely on morphological traits, their findings showed to be consistent with what molecular phylogenies have brought to the discussion regarding the relationships in Stephanopinae (Benjamin et al. 2008; Benjamin 2011; Wheeler et al. 2017). While few papers discussed the apparent "simplicity" of the copulatory features in the group, somatic characters (i.e., cryptic body coloration and rugose tegument usually with soil particles attached on it or associated lichen/fungi) have been constantly pointed out (Ramírez 2014; Machado et al. 2017; Machado \& Teixeira 2021). These traits and behaviors could be a result of an evolutionary convergence related to hunt and camouflage adaptations or a plesiomorphic condition in Thomisidae that could have changed multiple times along the phylogeny. Although these evolutionary hypotheses are still open, the importance of a tegument hue and ability of UV reflection were tested and seem to have an effect on the fitness of the spiders and their hunting success (Viera et al. 2017). In this sense, it is obvious that to better understand the evolution and relationships in Stephanopinae, performing extensive phylogenies is a must. But to do so, either to sequence the right material or to score a matrix of morphological characters, it is imperative that we start from an accurate taxonomic basis. This is part of an initial effort to clarify the morphological boundaries of some groups of Stephanopinae that were discussed in Machado \& Teixeira (2021). More genera are expected to be proposed in the near future since there are five other clades highlighted by these authors that are still in need of proper revisions.

\section{Acknowledgments}

The authors would like to thank to all dear colleagues and curators abovementioned for the specimens provided for this study. We are also thankful for the comments and suggestions made by the referees, especially to Dr Martín Ramírez, whose comments and suggestions have contributed to improve the quality and objectivity of the present work. We appreciate Thiago Carvalho for providing the images of live specimens of Kryptochroma gen. nov. The visit to MNHN collection was made possible by a CONICET fellowship for the study of European collections to Cristian J. Grismado, who is especially grateful to Christine Rollard and Elise-Anne Leguin for their invaluable help and assistance during his work in Paris. This study was financed in part by the Coordenação de Aperfeiçoamento de Pessoal de Nivel Superior - Brasil (CAPES) - Finance Code 001.

\section{References}

Benjamin S.P. 2011. Phylogenetics and comparative morphology of crab spiders (Araneae: Dionycha, Thomisidae). Zootaxa 3080: 1-108. https://doi.org/10.11646/zootaxa.3080.1.1

Benjamin S.P., Dimitrov D., Gillespie R.G. \& Hormiga G. 2008. Family ties: molecular phylogeny of crab spiders (Araneae: Thomisidae). Cladistics 24 (5): 708-722.

https://doi.org/10.1111/j.1096-0031.2008.00202.x

Keyserling E. 1880. Die Spinnen Amerikas, I. Laterigradae. Bauer and Raspe (E. Küster), Nürnberg. https://doi.org/10.5962/bhl.title.44813

Machado M. \& Teixeira R.A. 2021. Phylogenetic relationships in Stephanopinae: systematics of Stephanopis and Sidymella based on morphological characters (Araneae: Thomisidae). Organisms Diversity \& Evolution 21: 281-313. https://doi.org/10.1007/s13127-020-00472-x 
Machado M., Teixeira R.A. \& Lise A.A. 2015. Taxonomic notes on the crab spider genus Tobias Simon, 1895 (Araneae, Thomisidae, Stephanopinae). Zootaxa 4034: 565-576.

https://doi.org/10.11646/zootaxa.4034.3.8

Machado M., Teixeira R.A. \& Lise A.A. 2017. Cladistic analysis supports the monophyly of the Neotropical crab spider genus Epicadus and its senior synonymy over Tobias (Araneae: Thomisidae). Invertebrate Systematics 31: 442-455. https://doi.org/10.1071/IS16074

Machado M., Teixeira R.A. \& Lise A.A. 2018. There and back again: more on the taxonomy of the crab spiders genus Epicadus (Thomisidae: Stephanopinae). Zootaxa 4382: 501-530.

https://doi.org/10.11646/zootaxa.4382.3.4

Machado M., Guzati C., Viecelli R., Molina-Gómez D. \& Teixeira R.A. 2019a. A taxonomic review of the crab spider genus Sidymella (Araneae, Thomisidae) in the Neotropics. Zoosystematics and Evolution 95: 319-344. https://doi.org/10.3897/zse.95.34958

Machado M., Teixeira R.A. \& Milledge G.A. 2019b. On the Australian bark crab spiders genus Stephanopis: Taxonomic review and description of seven new species (Araneae: Thomisidae: Stephanopinae). Records of the Australian Museum 71: 217-276.

https://doi.org/10.3853/j.2201-4349.71.2019.1698

Mello-Leitão C.F. 1929. Aphantochilidas e Thomisidas do Brasil. Archivos do Museu Nacional do Rio de Janeiro 31: 1-359.

Mello-Leitão C.F. 1942. Arañas del Chaco y Santiago del Estero. Revista del Museo de La Plata 2: 381-426.

Prado A.W., Baptista R.L.C. \& Machado M. 2018. Taxonomic review of Epicadinus Simon, 1895 (Araneae: Thomisidae). Zootaxa 4459: 201-234. https://doi.org/10.11646/zootaxa.4459.2.1

Ramírez M.J. 2014. The morphology and phylogeny of Dionychan spiders (Araneae: Araneomorphae). Bulletin of the American Museum of Natural History 2014 (390): 1-374. https://doi.org/10.1206/821.1

Silva-Moreira T. \& Machado M. 2016. Taxonomic revision of the crab spider genus Epicadus Simon, 1895 (Arachnida: Araneae: Thomisidae) with notes on related genera of Stephanopinae Simon, 1895. Zootaxa 4147: 281-310. https://doi.org/10.11646/zootaxa.4147.3.4

Simon E. 1895. Histoire naturelle des araignées. Deuxième édition. Librairie Encyclopédique de Roret, Paris. https://doi.org/10.5962/bhl.title.51973

Soares B.A.M. \& Soares H.E.M. 1946. Contribuição ao estudo das aranhas do estado do Espírito Santo. Papéis Avulsos do Departamento de Zoologia 7: 51-72.

Taczanowski L. 1872. Les Aranéides de la Guyane française. Horae Societatis Entomologicae Rossicae 9: 64-150, 261-286.

Teixeira R.A., Campos L.A. \& Lise A.A. 2014. Phylogeny of Aphantochilinae and Strophiinae sensu Simon (Araneae; Thomisidae). Zoologica Scripta 43 (1): 65-78. https://doi.org/10.1111/zsc. 12036

Vieira C., Ramires E.N., Vasconcellos-Neto J., Poppi R.J. \& Romero G.Q. 2017. Crab spider lures prey in flowerless neighborhoods. Scientific Reports 7: 1-7. https://doi.org/10.1038/s41598-017-09456-y

Walckenaer C.A. 1837. Histoire naturelle des insectes. Aptères. Tome premier. Roret, Paris. https://doi.org/10.5962/bhl.title.61095

Wheeler W.C., Coddington J.A., Crowley L.M., Dimitrov D., Goloboff P.A., Griswold C.E., Hormiga G., Prendini L., Ramírez M.J., Sierwald P., Almeida-Silva L., Álvarez-Padilla F., Arnedo M.A., Benavides Silva L.R., Benjamin S.P., Bond J.E., Grismado C.J., Hasan E., Hedin M., Izquierdo M.A., Labarque F.M., Ledford J., Lopardo L., Maddison W.P., Miller J.A., Piacentini L.N., Platnick N.I., 
Polotow D., Silva-Dávila D., Scharff N., Szuts T., Ubick D., Vink C.J., Wood H.M. \& Zhang J. 2016. The spider tree of life: phylogeny of Araneae based on target-gene analyses from an extensive taxon sampling. Cladistics 33: 574-616. https://doi.org/10.1111/cla.12182

Manuscript received: 29 April 2021

Manuscript accepted: 30 September 2021

Published on: 3 November 2021

Section editor: Rudy Jocqué

Desk editor: Pepe Fernández

Printed versions of all papers are also deposited in the libraries of the institutes that are members of the EJT consortium: Muséum national d'histoire naturelle, Paris, France; Meise Botanic Garden, Belgium; Royal Museum for Central Africa, Tervuren, Belgium; Royal Belgian Institute of Natural Sciences, Brussels, Belgium; Natural History Museum of Denmark, Copenhagen, Denmark; Naturalis Biodiversity Center, Leiden, the Netherlands; Museo Nacional de Ciencias Naturales-CSIC, Madrid, Spain; Real Jardín Botánico de Madrid CSIC, Spain; Zoological Research Museum Alexander Koenig, Bonn, Germany; National Museum, Prague, Czech Republic. 